\title{
Xylocarpins A-I, Limonoids from Chinese Mangrove Plant Xylocarpus granatum
}

\author{
Jianxin Cui, Jun Wu, Zhiwei Deng, Peter Proksch, Wenhan Lin*
}

State Key Laboratory of Natural and Biomimetic Drugs, School of Pharmaceutical

Science, Peking University, Beijing 100083, China; Guangdong Key Laboratory of

Marine Materia Medica, Chinese Academy of Sciences, Guangzhou 510301, China;

Analysis and Test Center, Beijing Normal University, Beijing 100073, China; Institute of Pharmaceutical Biology, Heinrich-Heine University, 40225 Duesseldorf, Germany

* To whom correspondence should be addressed. Tel.: +86-10-82806188; Fax:

+86-10-82802724. E-mail address: whlin@bjmu.edu.cn (W. Lin) 


\section{Table of content}

Figure 1 Structures of xylocarpins A-I (1-5, 7-10)

1. Xylocarpin A (1)

IR spectrum

${ }^{1} \mathrm{H}$ NMR spectrum

${ }^{13} \mathrm{C}$ and DEPT NMR spectra

HMQC spectrum

HMBC spectrum

NOESY spectrum

DQFCOSY spectrum

HRSIMS data

2 Xylocarpin B (2)

IR spectrum

${ }^{1} \mathrm{H}$ NMR spectrum

${ }^{13} \mathrm{C}$ NMR spectrum

HMQC spectrum

HMBC spectrum

NOESY spectrum

DQFCOSY spectrum

\section{HRSIMS data}

3 Xylocarpin C (3)

IR spectrum

${ }^{1} \mathrm{H}$ NMR spectrum

${ }^{13} \mathrm{C}$ and DEPT spectra

HMQC spectrum

HMBC spectrum

NOESY spectrum

ESIMS spectrum

HRSIMS data

4 Xylocarpin D (4)

IR spectrum

${ }^{1} \mathrm{H}$ NMR spectrum

${ }^{13} \mathrm{C}$ and DEPT spectra

HMQC spectrum 
HMBC spectrum

DQFCOSY spectrum

ESIMS spectrum

HRSIMS data

5 Xylocarpin E (5)

IR spectrum

${ }^{1} \mathrm{H}$ NMR spectrum

${ }^{13} \mathrm{C}$ and DEPT spectra

HMQC spectrum

HMBC spectrum

DQFCOSY spectrum

ESIMS spectrum

HRSIMS data

6 6-Dehydroxyxylocarpin D (6)

IR spectrum

${ }^{1} \mathrm{H}$ NMR spectrum

${ }^{13} \mathrm{C}$ and DEPT spectra

HMQC spectrum

HMBC spectrum

NOESY spectrum

ESIMS spectrum

HRSIMS data

7 Xylocarpin F (7)

IR spectrum

${ }^{1} \mathrm{H}$ NMR spectrum

${ }^{13} \mathrm{C}$ and DEPT spectra

HMQC spectrum

HMBC spectrum

NOESY spectrum

ESIMS spectrum

HRSIMS data

8 Xylocarpin G (8)

IR spectrum

${ }^{1} \mathrm{H}$ NMR spectrum 
${ }^{13} \mathrm{C}$ and DEPT spectra

HMQC spectrum

HMBC spectrum

HRSIMS data

\section{Xylocarpin $\mathbf{H}(9)$}

IR spectrum

${ }^{1} \mathrm{H}$ NMR spectrum

${ }^{13} \mathrm{C}$ and DEPT spectra

HMQC spectrum

HMBC spectrum

NOESY spectrum

ESIMS spectrum

HRSIMS data

10 Xylocarpin I (10)

IR spectrum

${ }^{1} \mathrm{H}$ NMR spectrum

${ }^{13} \mathrm{C}$ and DEPT spectra

HMQC spectrum

HMBC spectrum

NOESY spectrum

ESIMS spectrum

HRSIMS data 


\section{Structures of xylocarpins A-I (1-5, 7-10)}
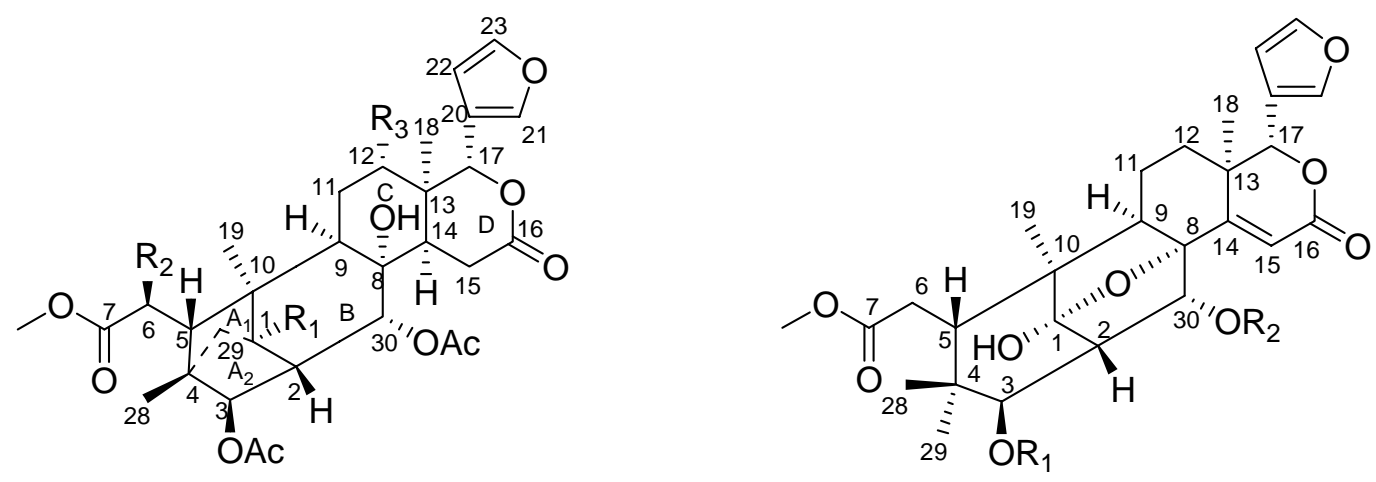

1. $R_{1}=R_{2}=O A C, R_{3}=H$

2. $R_{1}=O A C, R_{2}=R_{3}=H$

3. $\mathrm{R}_{1}=\mathrm{OH}, \mathrm{R}_{2}=\mathrm{H}, \mathrm{R}_{3}=\mathrm{OAc}$

4. $R_{1}=R_{3}=O A C, R_{2}=O H$

5. $\mathrm{R}_{1}=\mathrm{R}_{2}=\mathrm{OAC}, \mathrm{R}_{3}=\mathrm{OH}$

6. $R_{1}=R_{3}=$ OAc, $R_{2}=H$

7. $R_{1}=R_{2}=A c$

8. $R_{1}=O A c, R_{2}=$ Tig

Tig $=\prod_{O}^{5}$
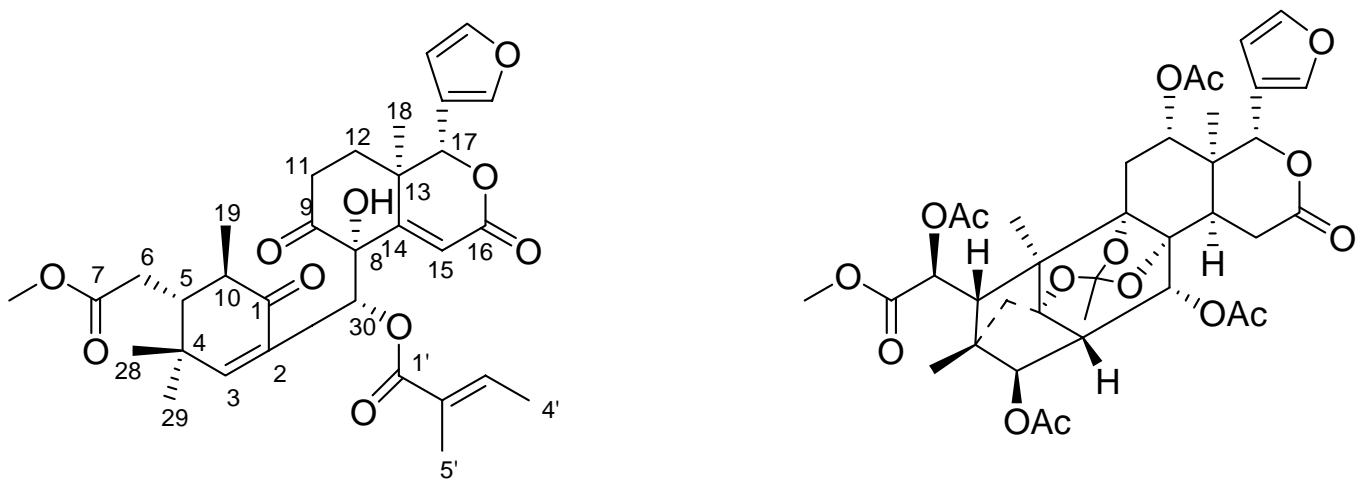

9

10 


\section{Xylocarpin A (1)}

IR spectrum

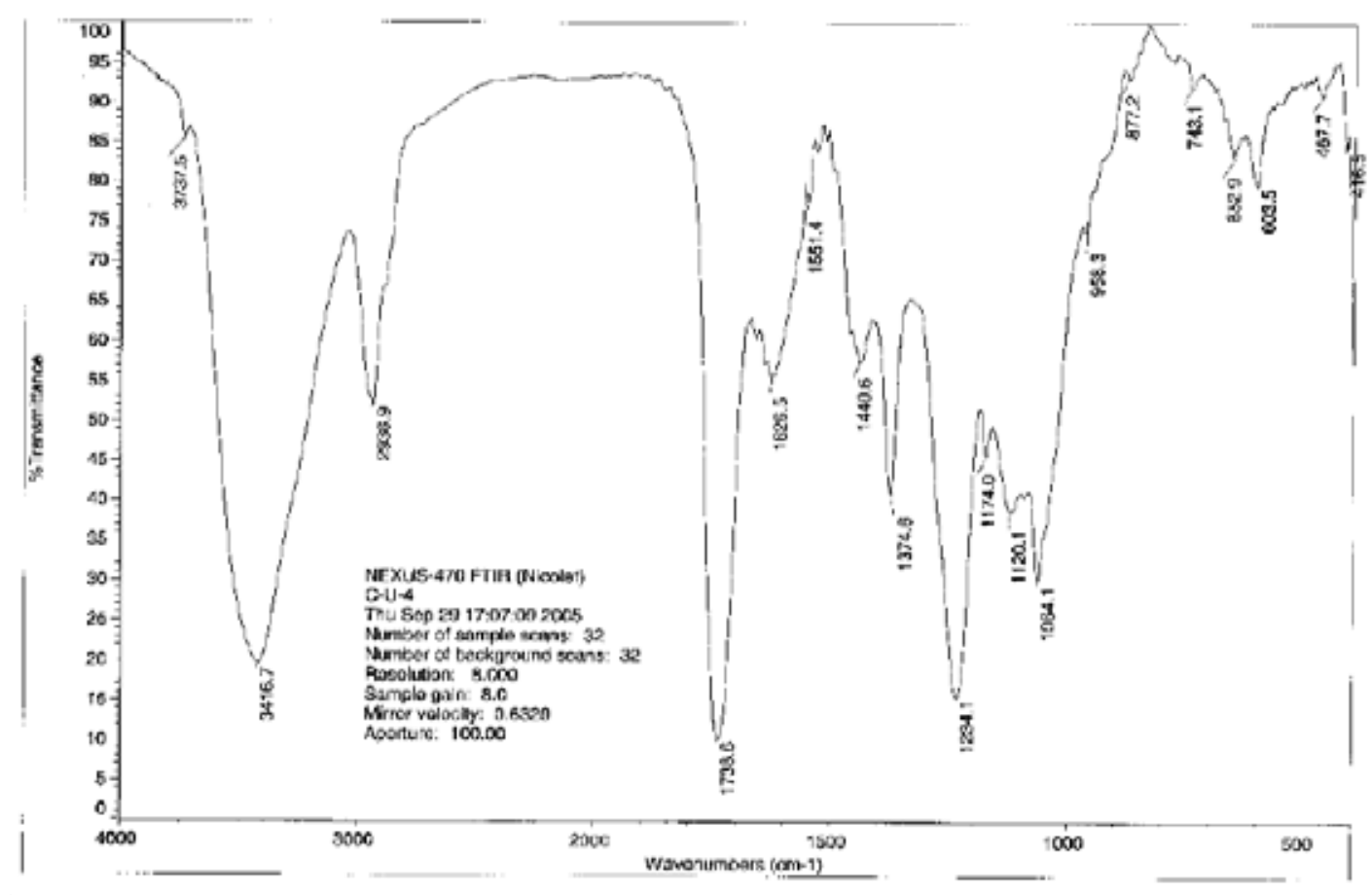

\section{${ }^{1}$ H NMR spectrum}

Avance 500 Bruker. AST Center BNU
Samole: $c-U-4$. Solvent: cdc13

Sample: c-U-4. Solvent: $\operatorname{cdc} 13$
Spectrun: cul-9 41 qc

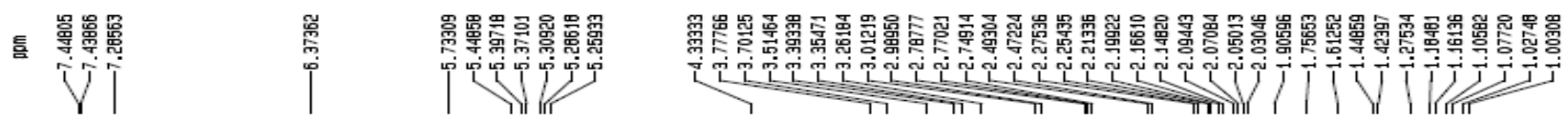

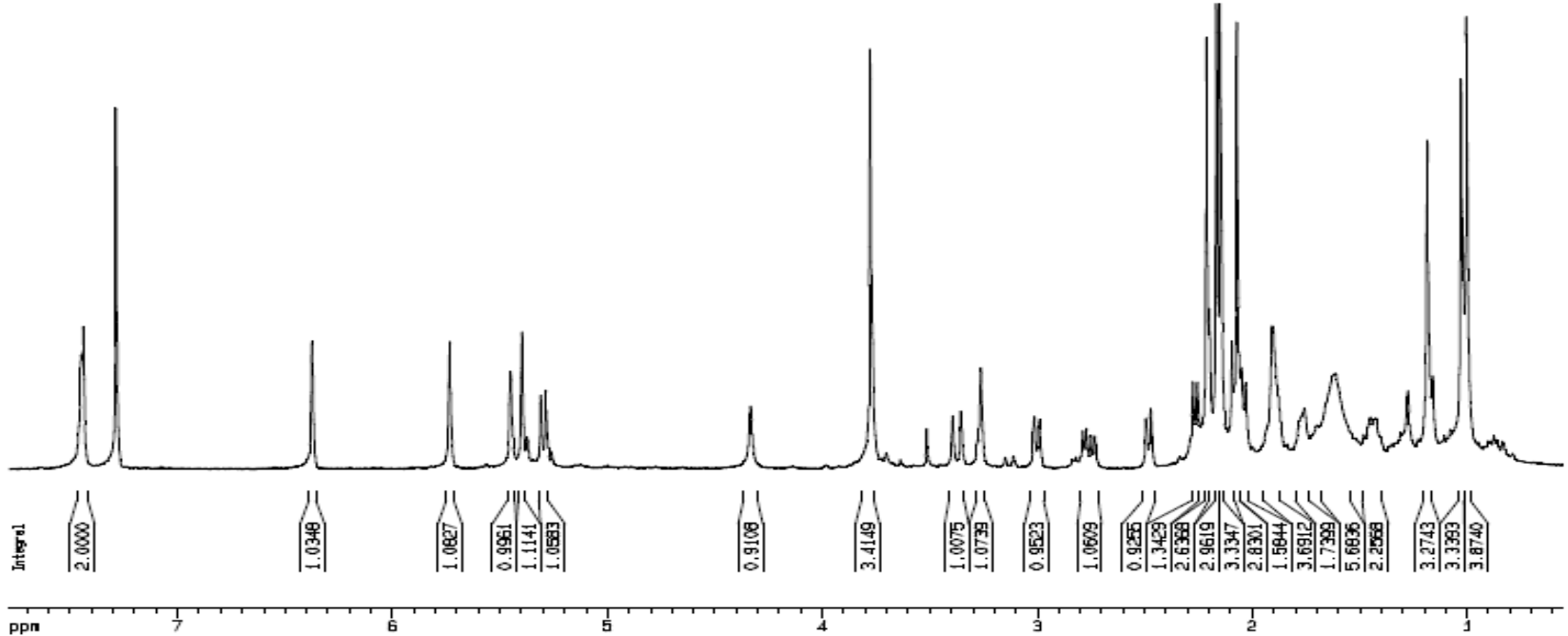




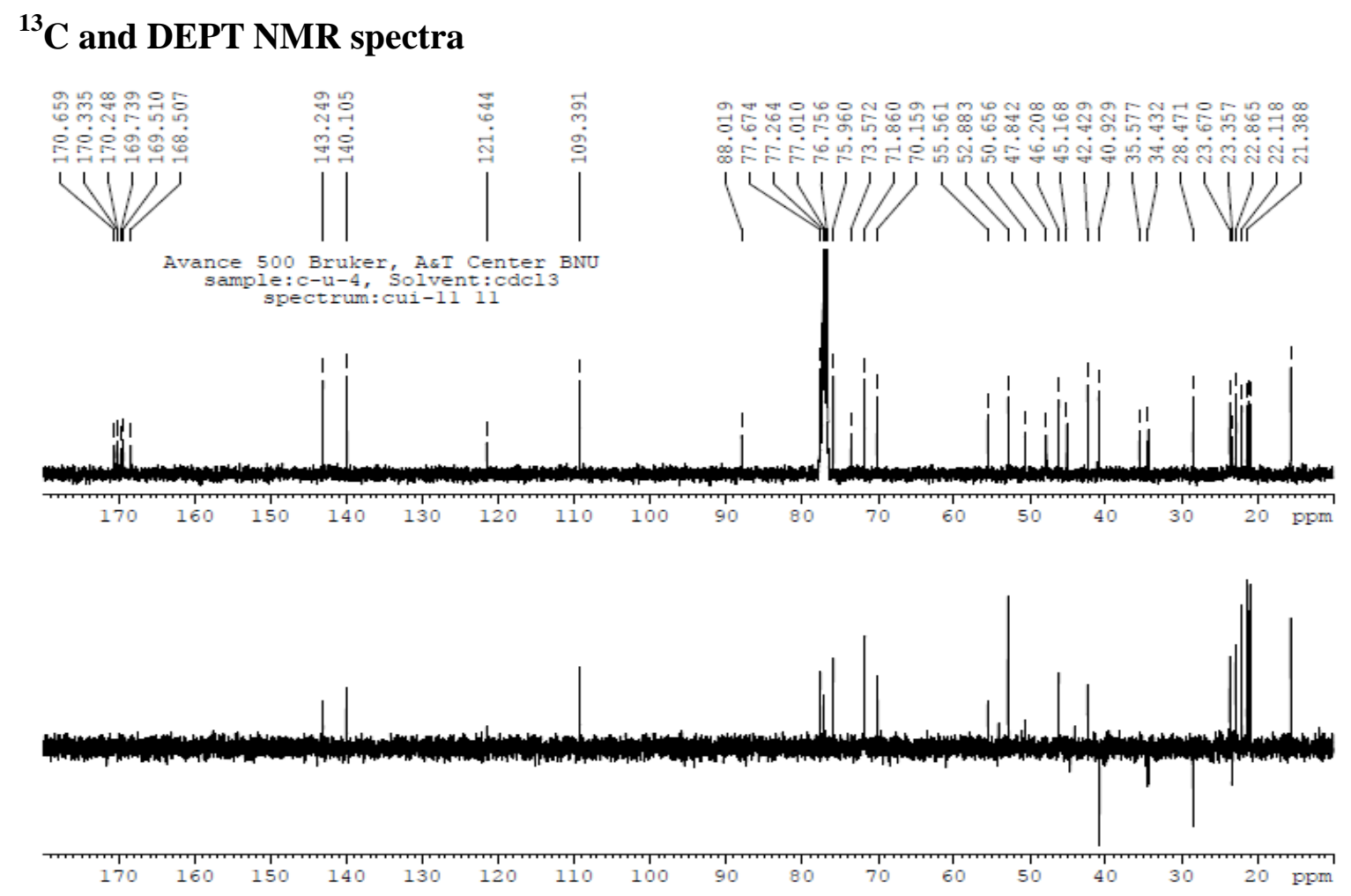

\section{HMQC spectrum}

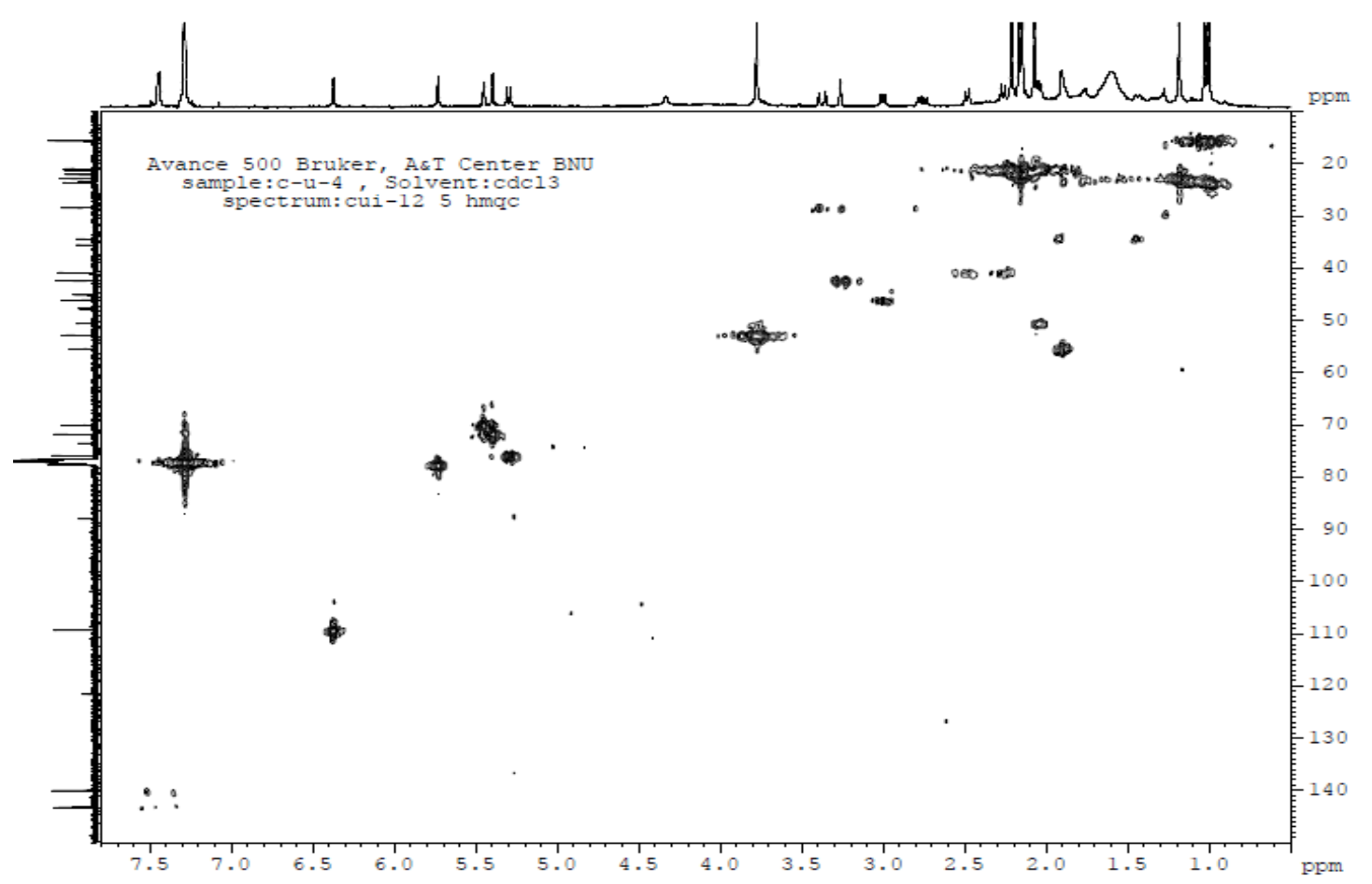




\section{HMBC spectrum}

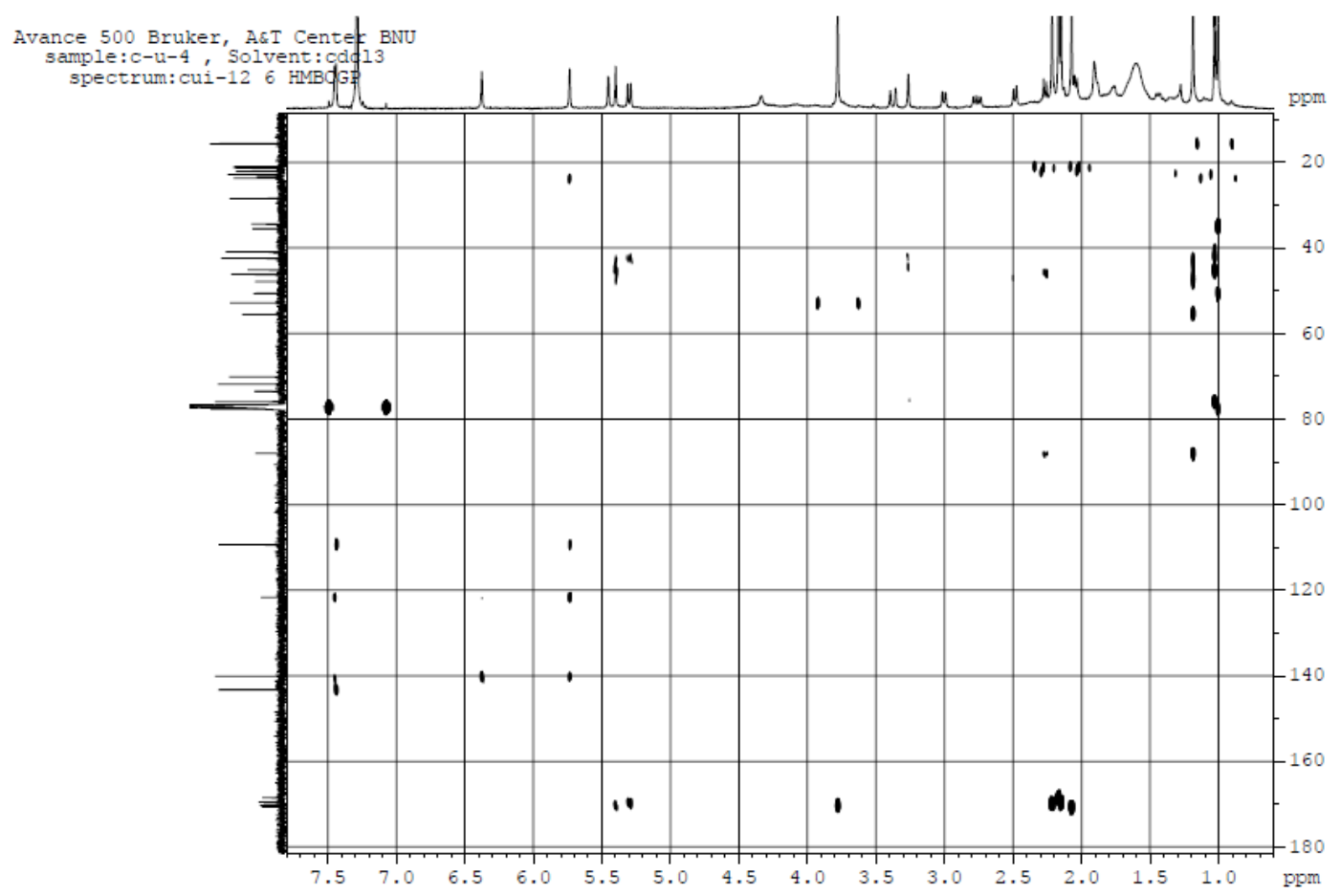

DQFCOSY spectrum

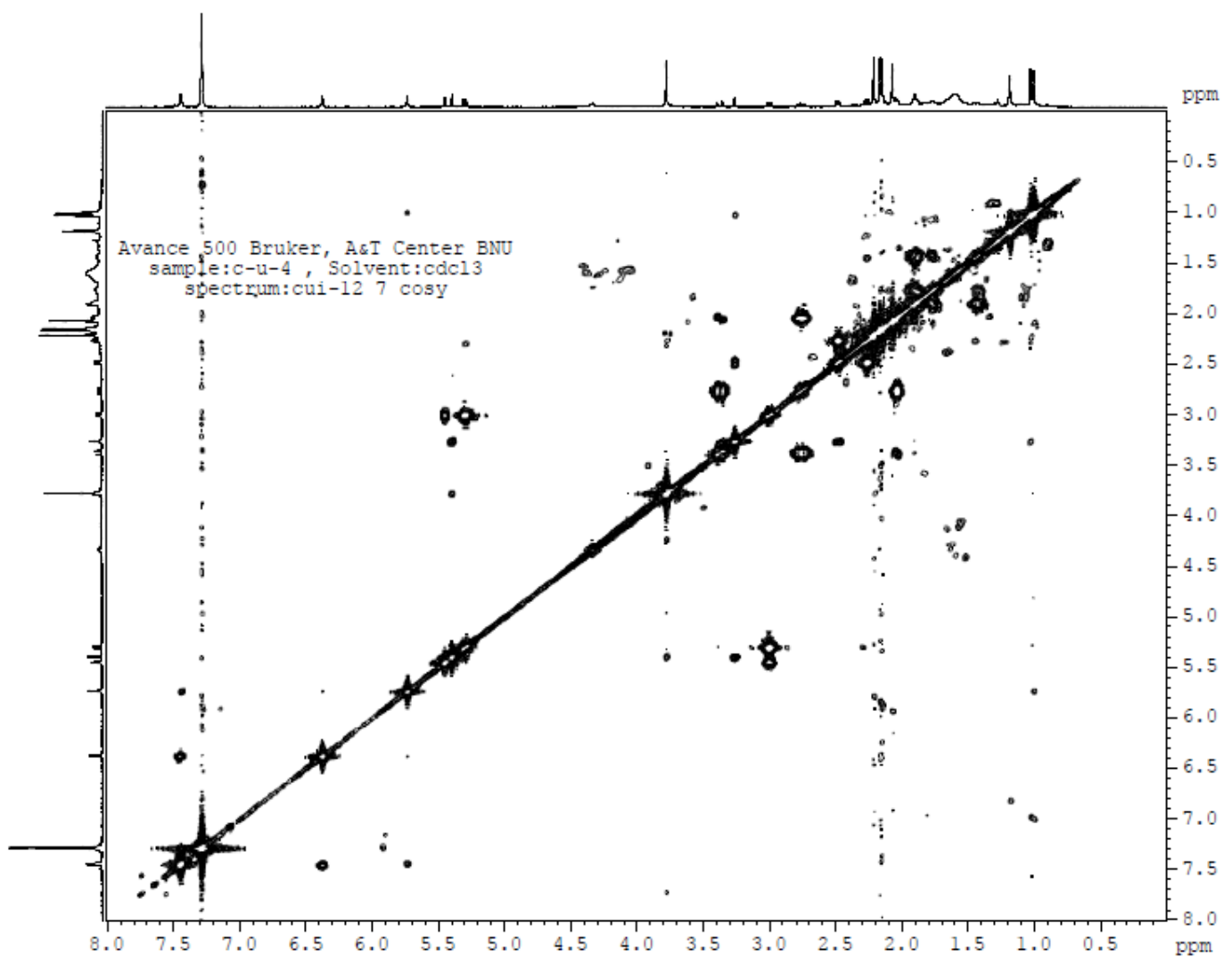




\section{NOESY spectrum}

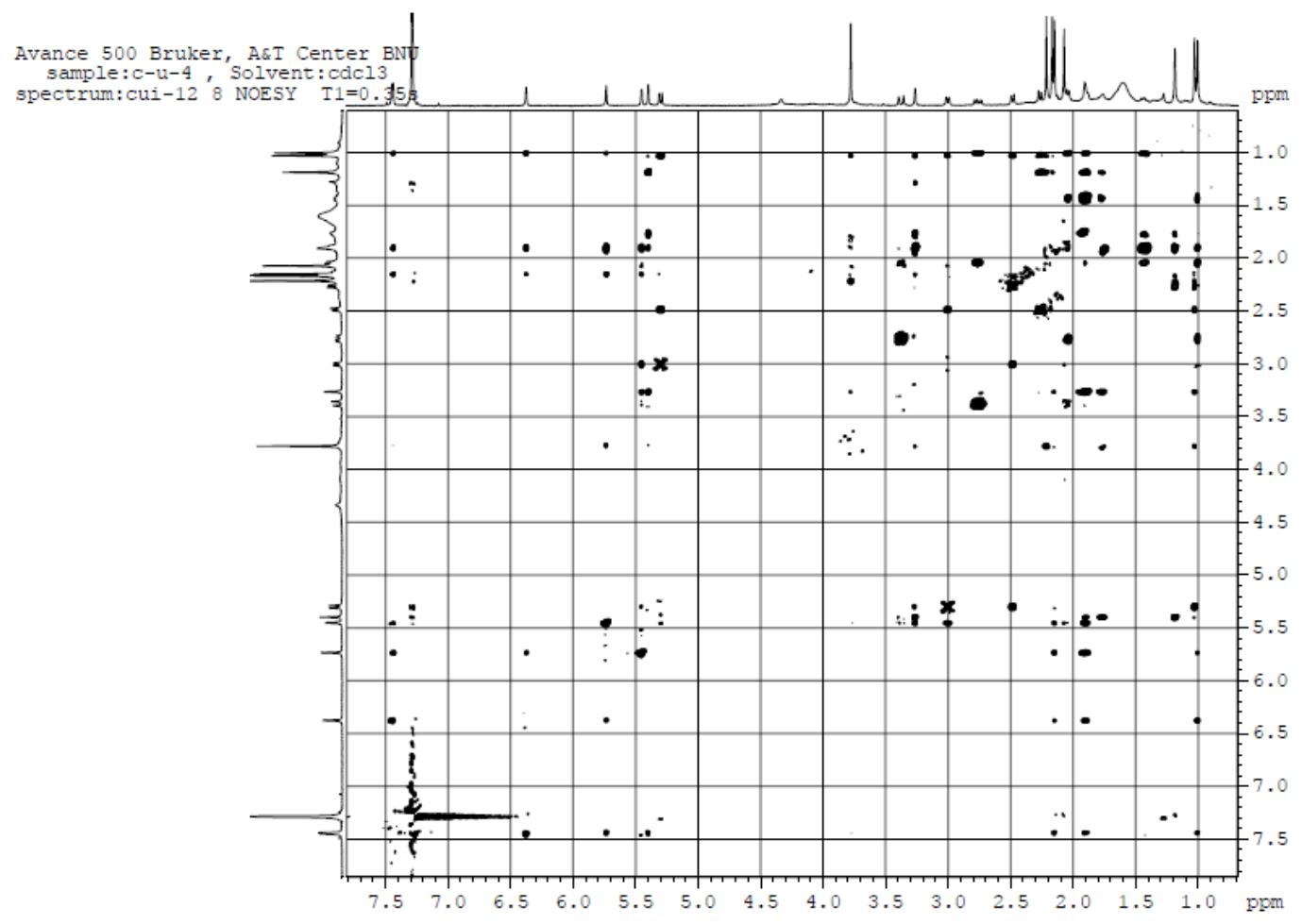

\section{HRSIMS data}

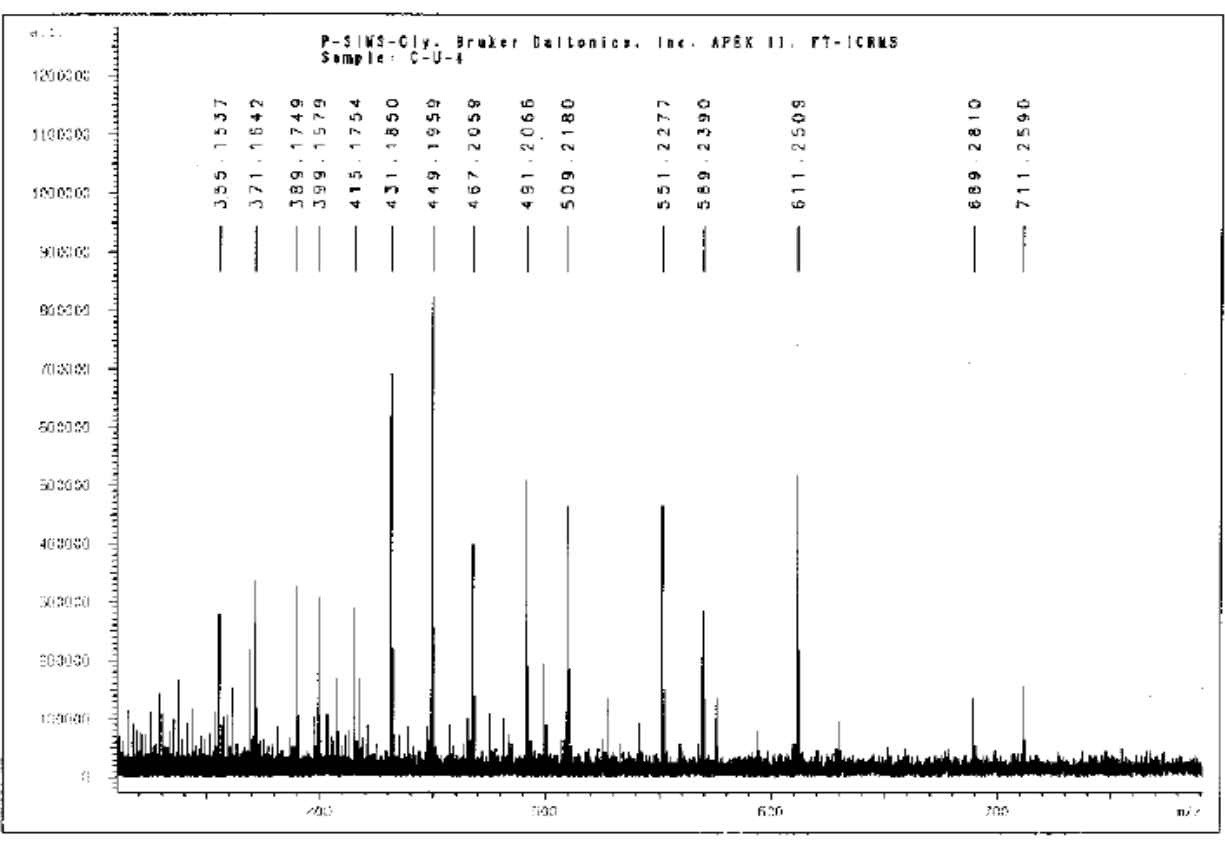




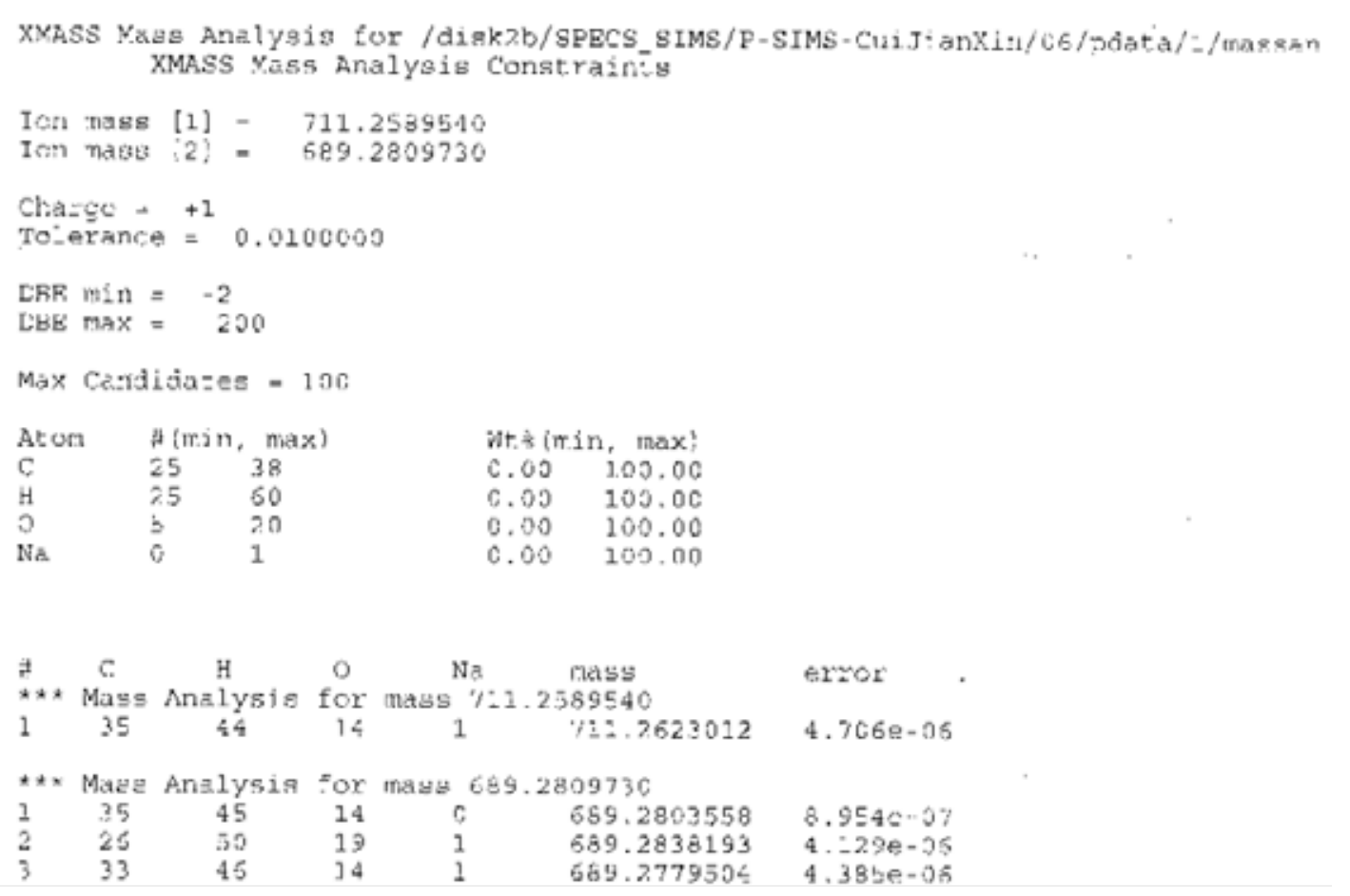

\section{Xylocarpin B (2)}

IR spectrum

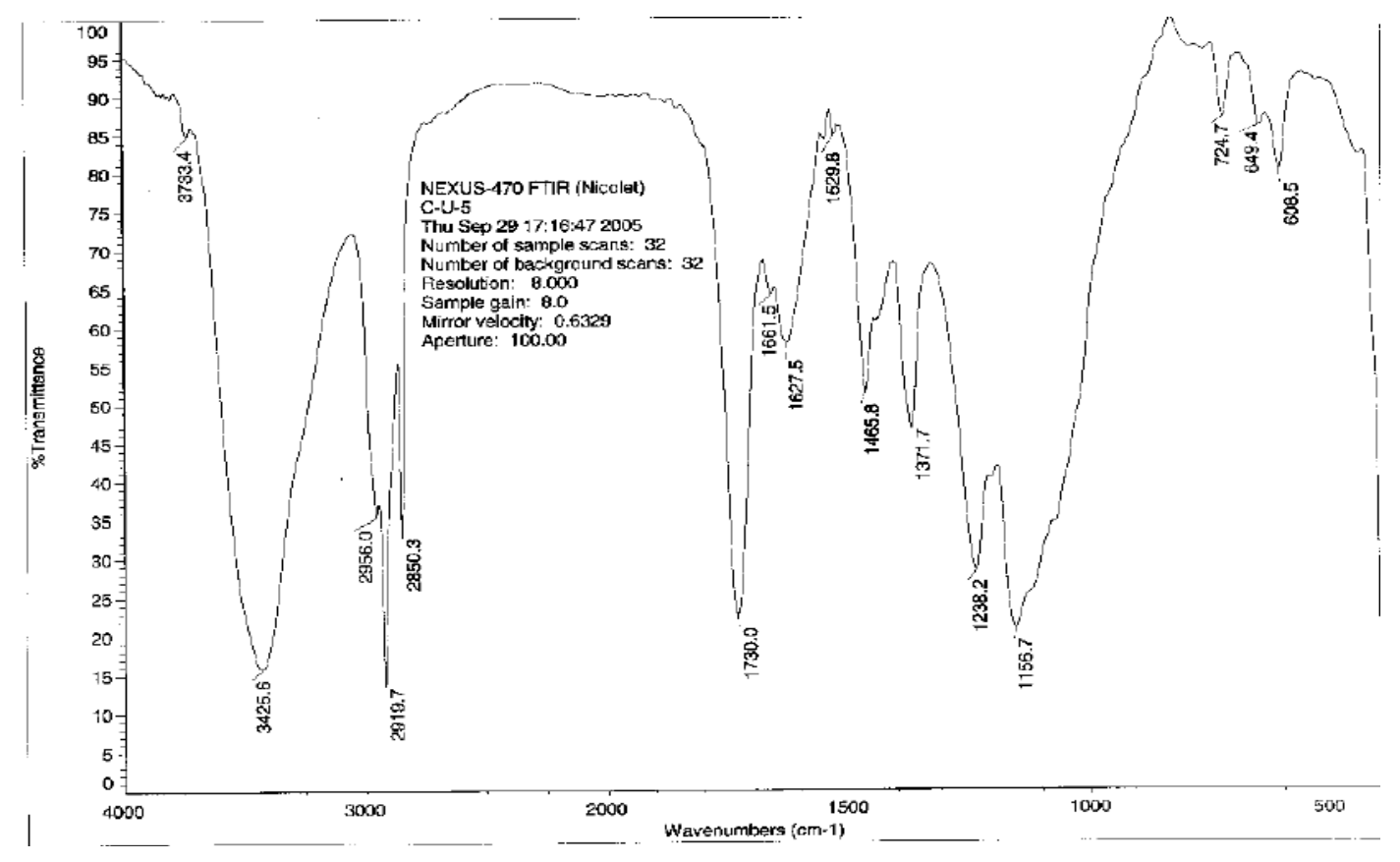




\section{${ }^{1}$ H NMR spectrum}

Avance 500 Bruker, AGT Center BNu
sample: $c-u-5$, Solvent: edc13

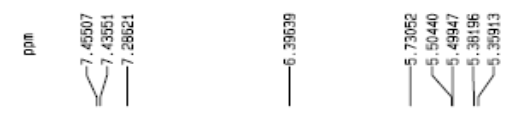

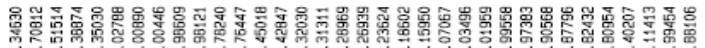

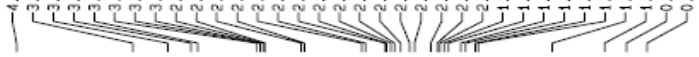

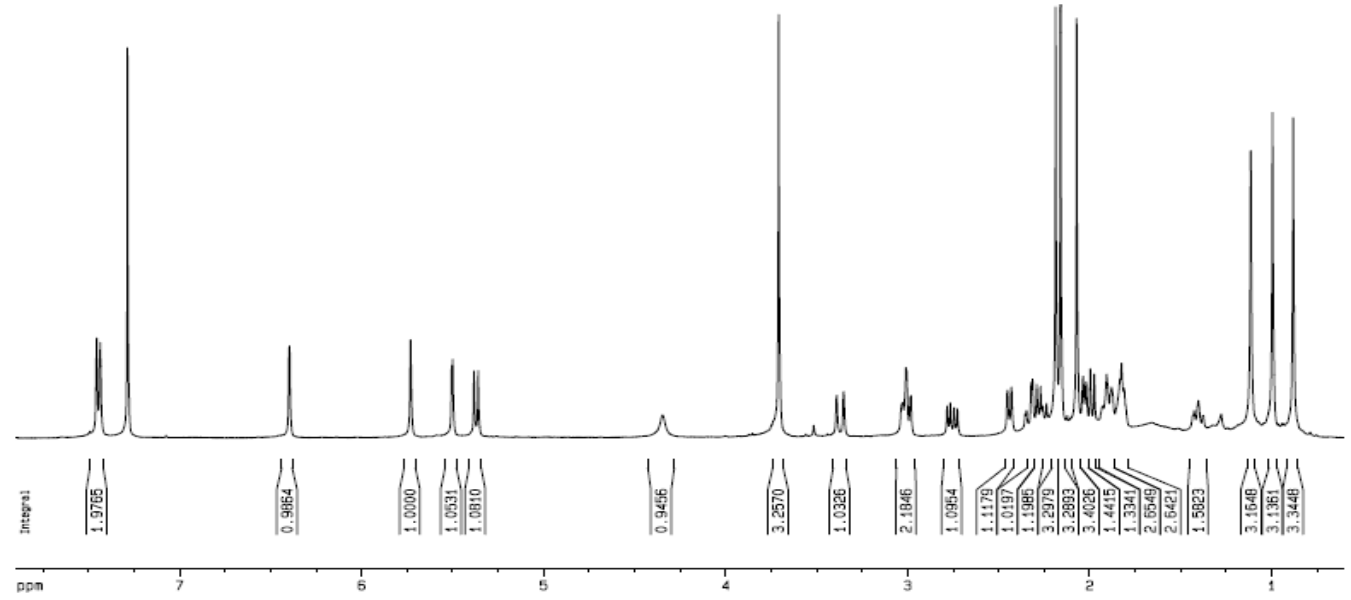

\section{${ }^{13} \mathrm{C}$ NMR spectrum}

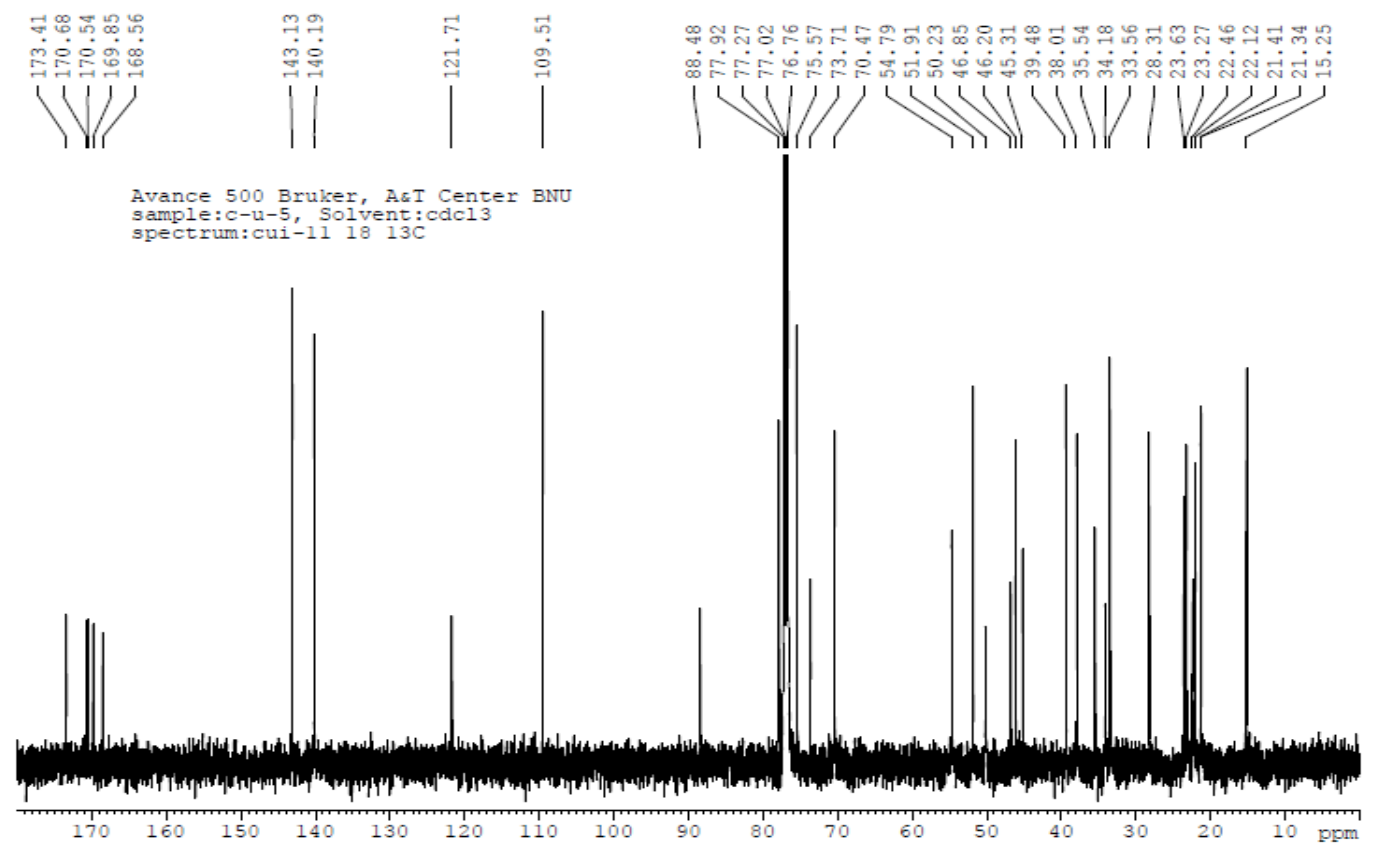




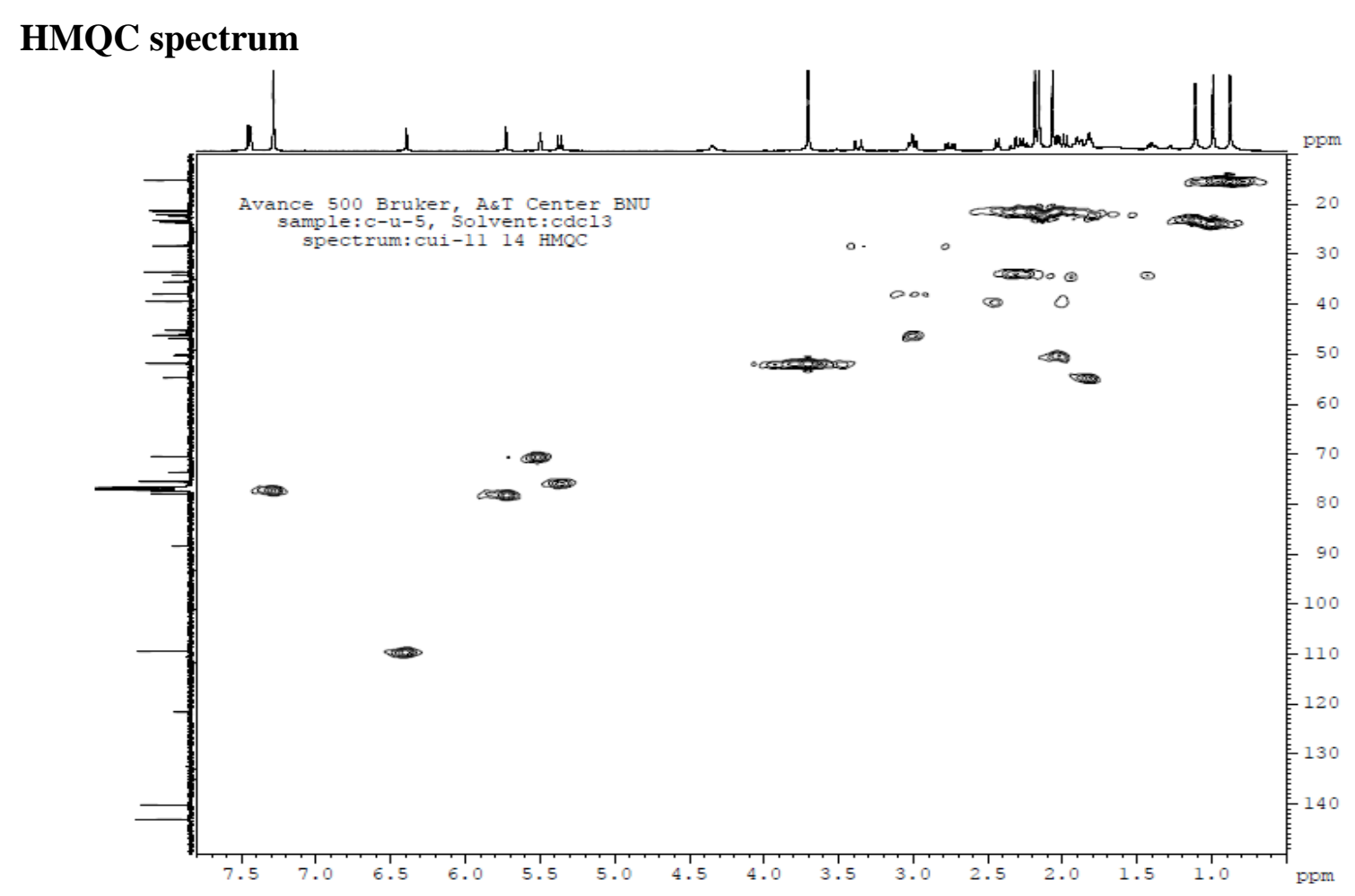

\section{HMBC spectrum}

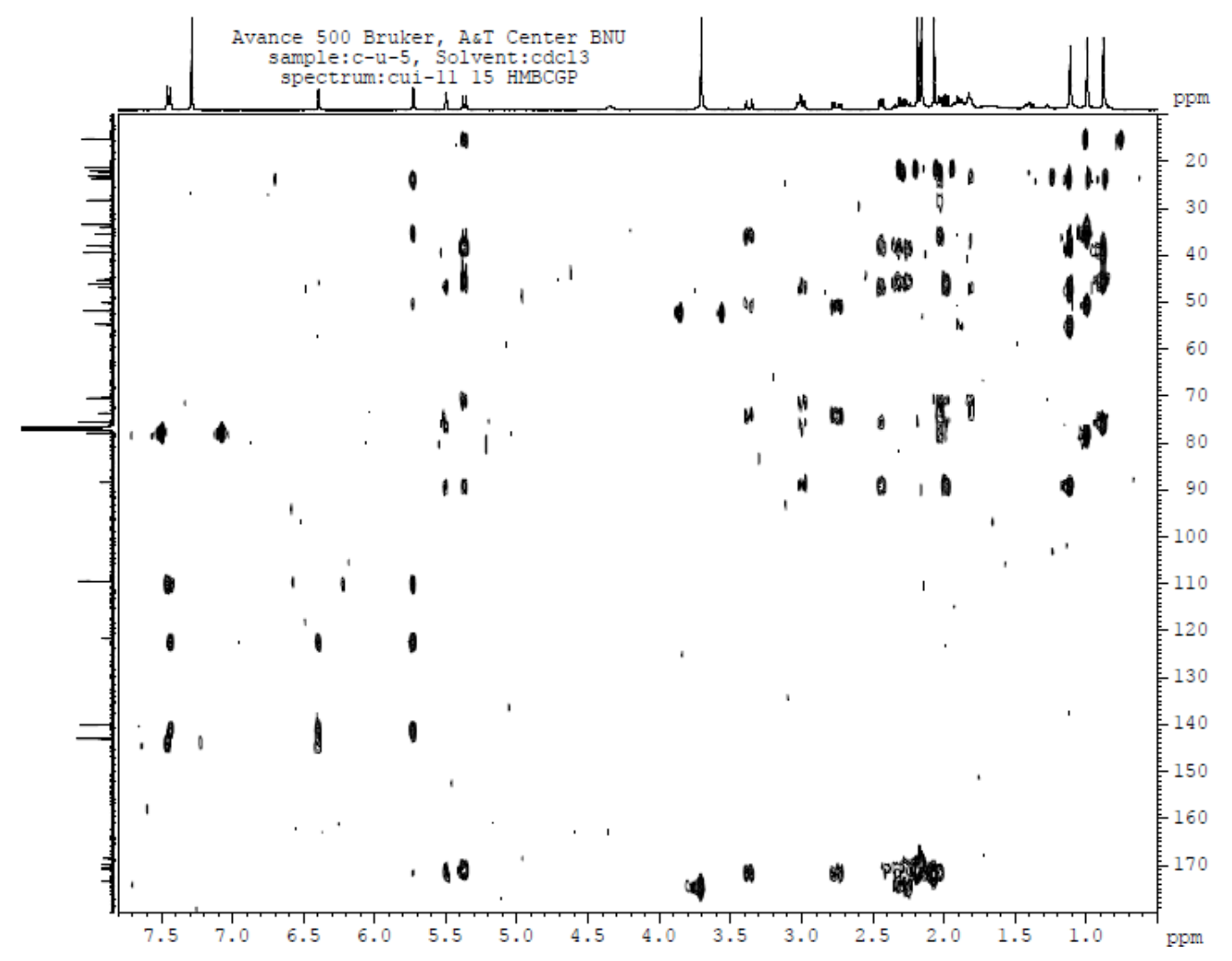




\section{NOESY spectrum}

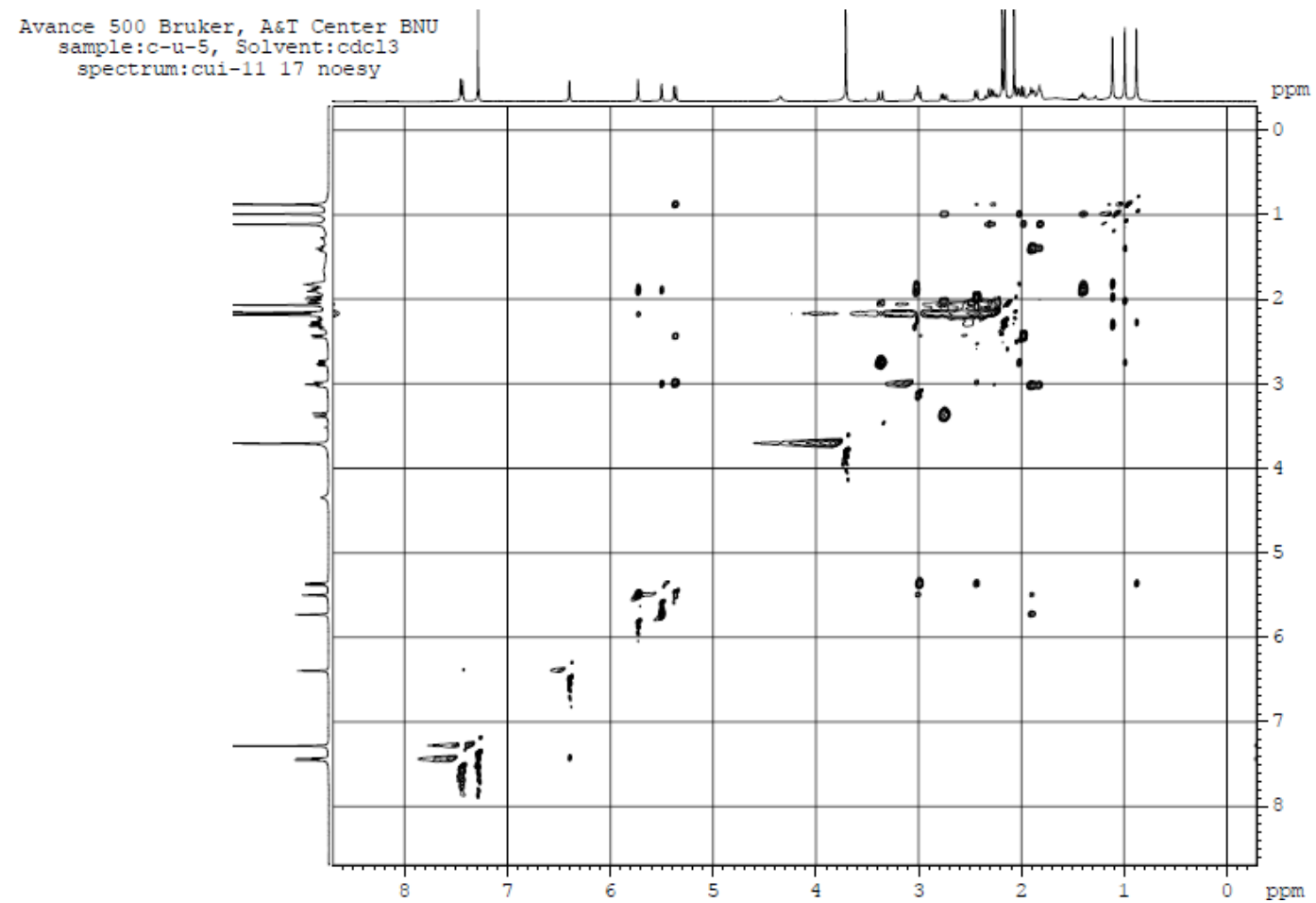

\section{DQFCOSY spectrum}

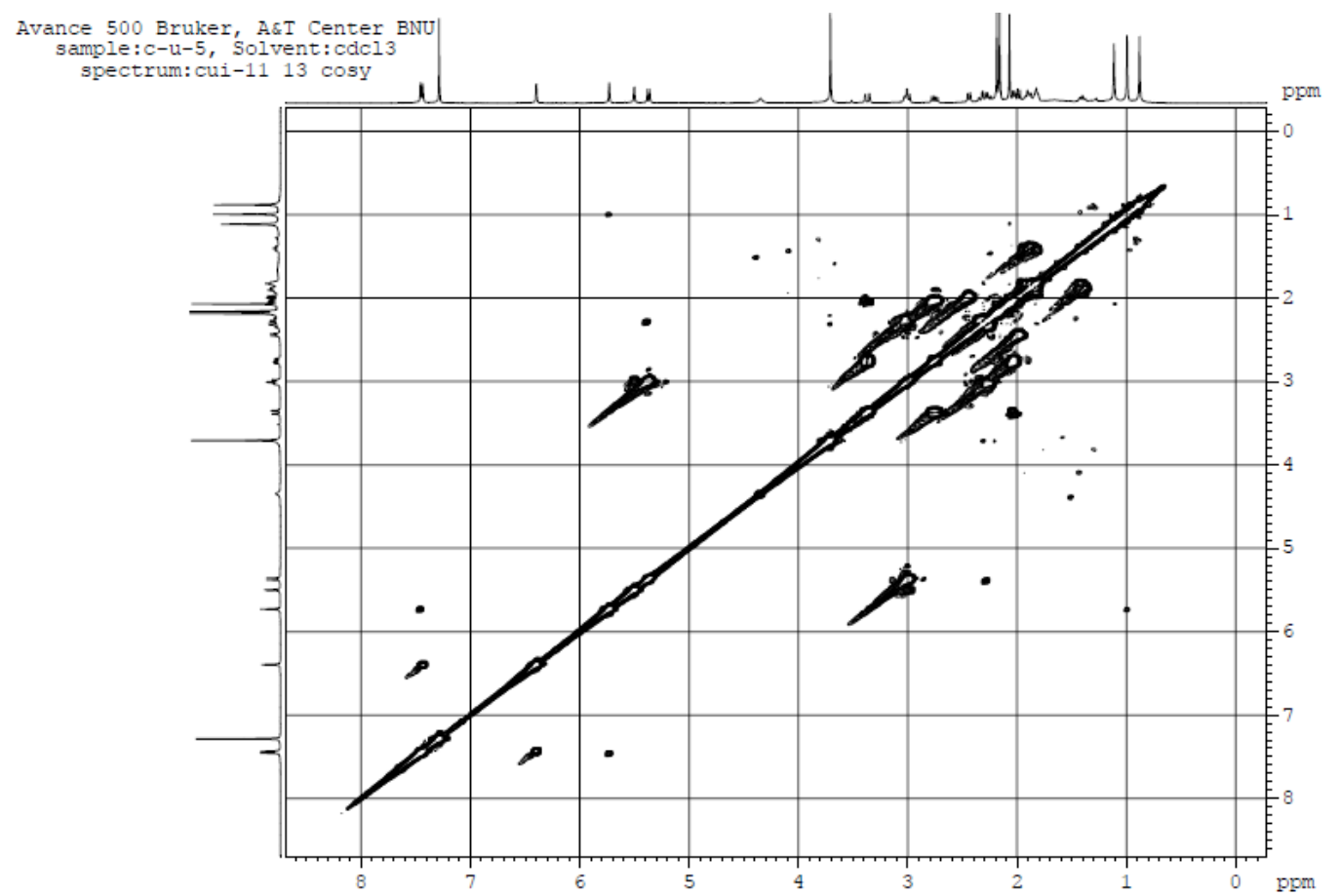




\section{HRSIMS data}

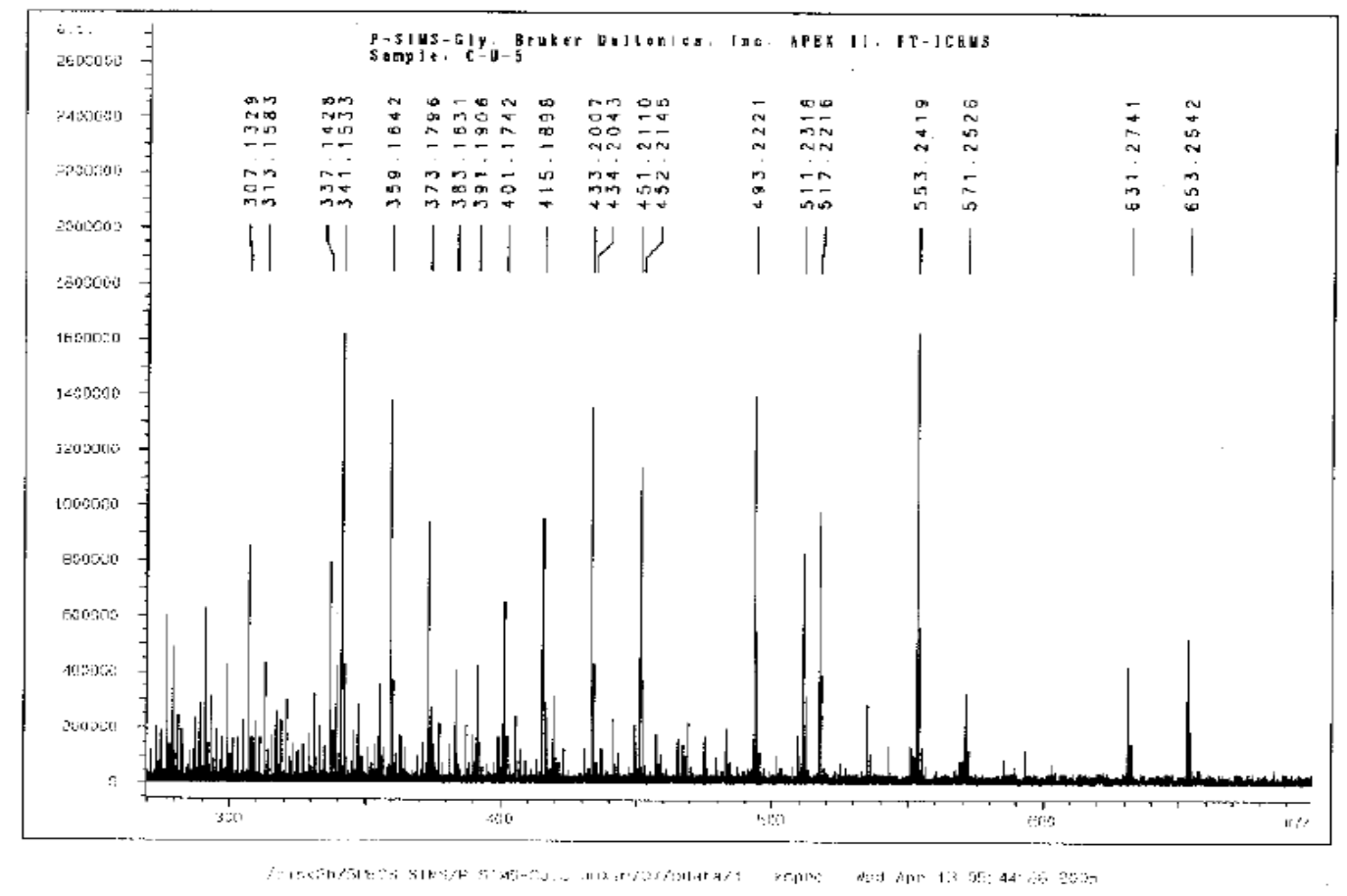

XMAss Mass Analysis for /disk2b/SPECS_SIMS/P-SIMS-Cui-̃ar.Xin/07/pdat.a/1/ma
XMASS Mass Analysia Constraints

Ior: mass $[:]=653.25422] \mathrm{C}$

Ton mass $[\ddot{z}]=631.2741490$

Charge $=+1$

To-eranse $=0.0100000$

DSE MIn $=-2$

DEE $\operatorname{nax}=2 \mathrm{CO}$

Max Cafidiates - -co

$\begin{array}{lllll}\lambda=c m & \#(\min , \max ) & \text { Wt } 8(\min , \max ) \\ 6 & 25 & 38 & 0.00 & 100.00 \\ H & 25 & 60 & 0.00 & 100.0 \mathrm{C} \\ 0 & 5 & 2 \mathrm{C} & 0.00 & 100.00 \\ \mathrm{Na} & 0 & 1 & 0.00 & 300.00\end{array}$

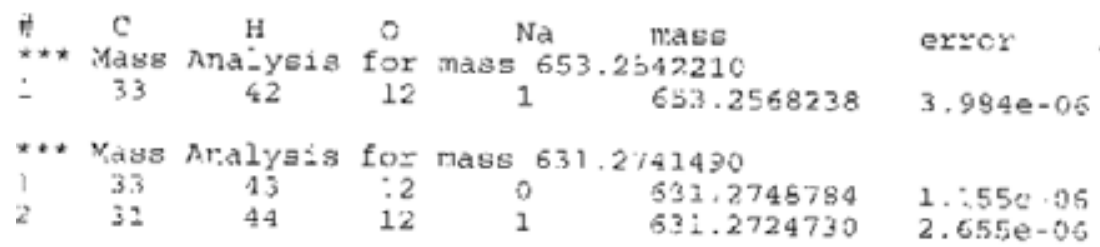




\section{Xylocarpin C (3)}

IR spectrum

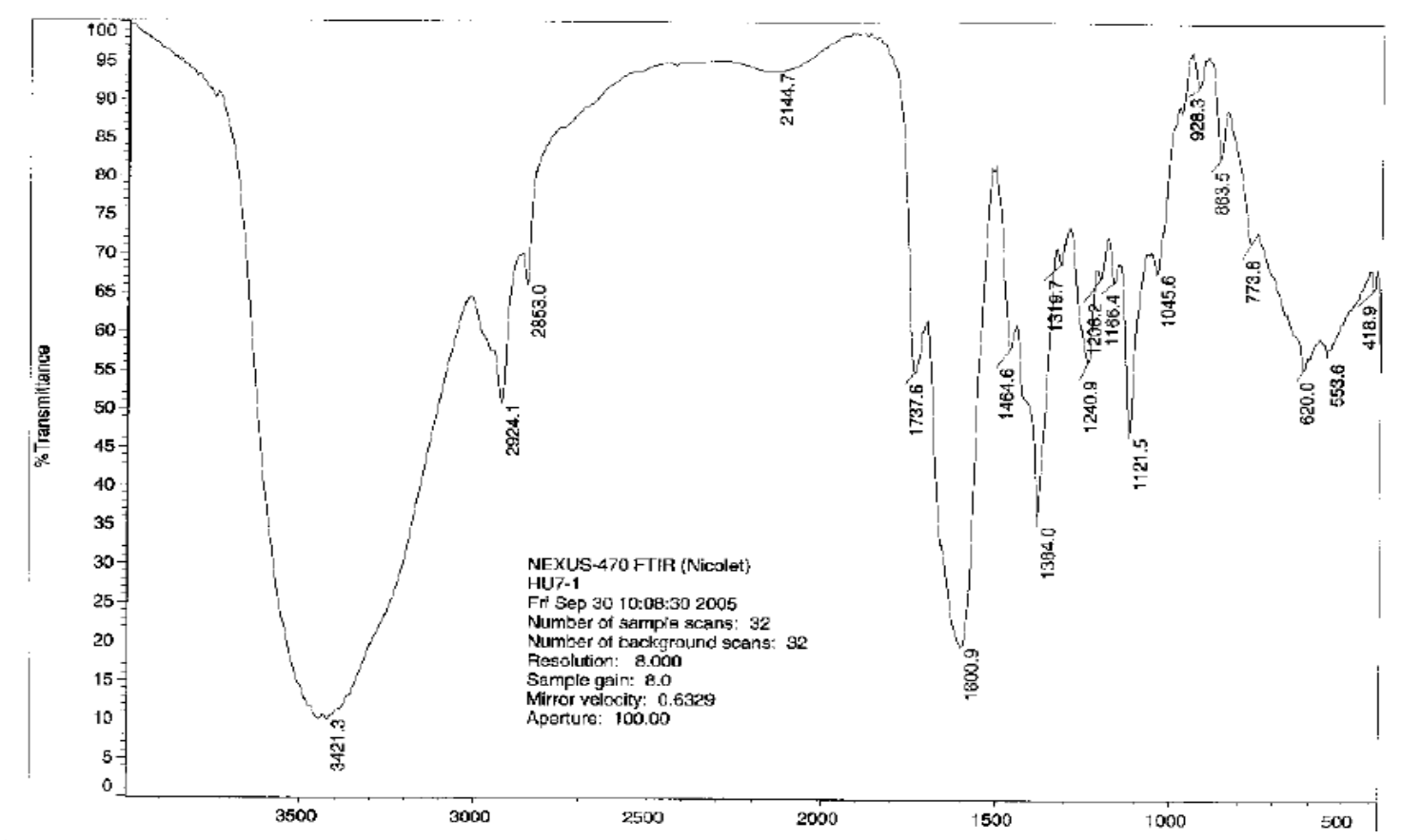

\section{${ }^{1}$ H NMR spectrum}

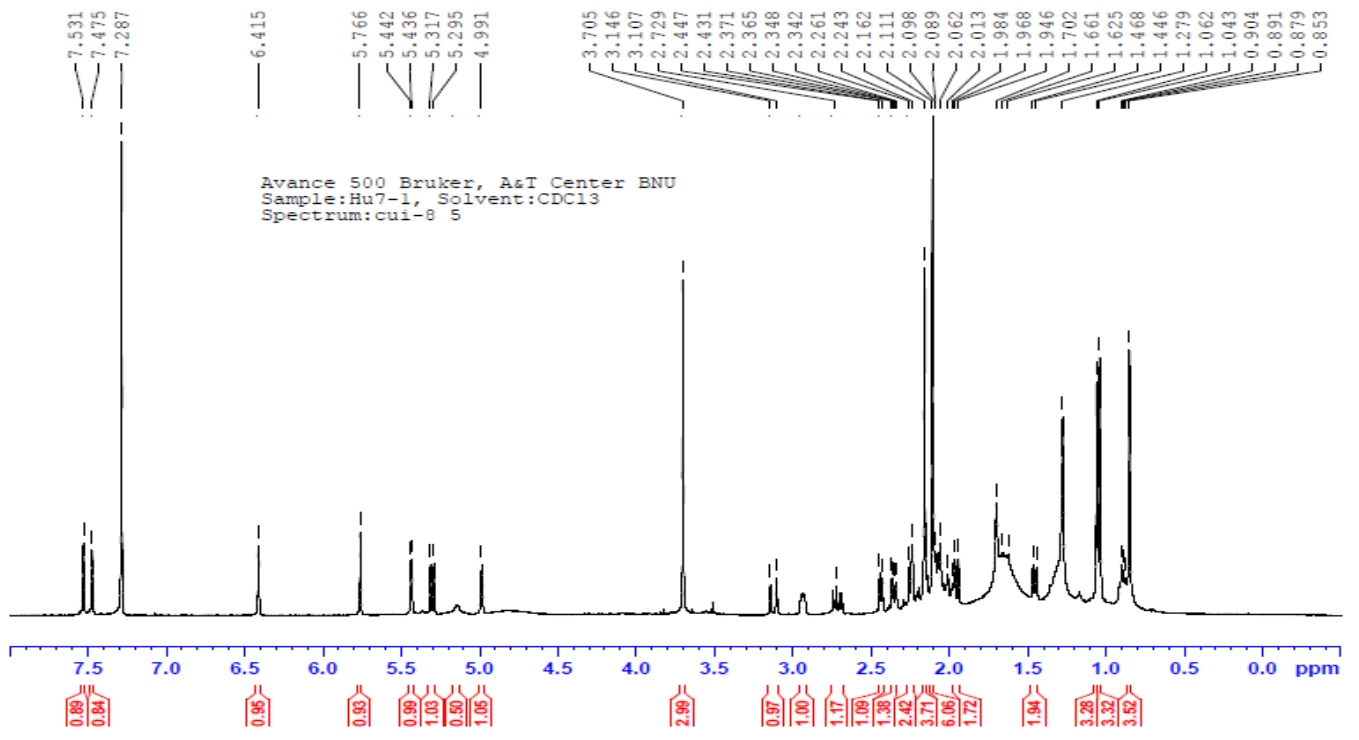




\section{${ }^{13} \mathrm{C}$ and DEPT NMR spectra}

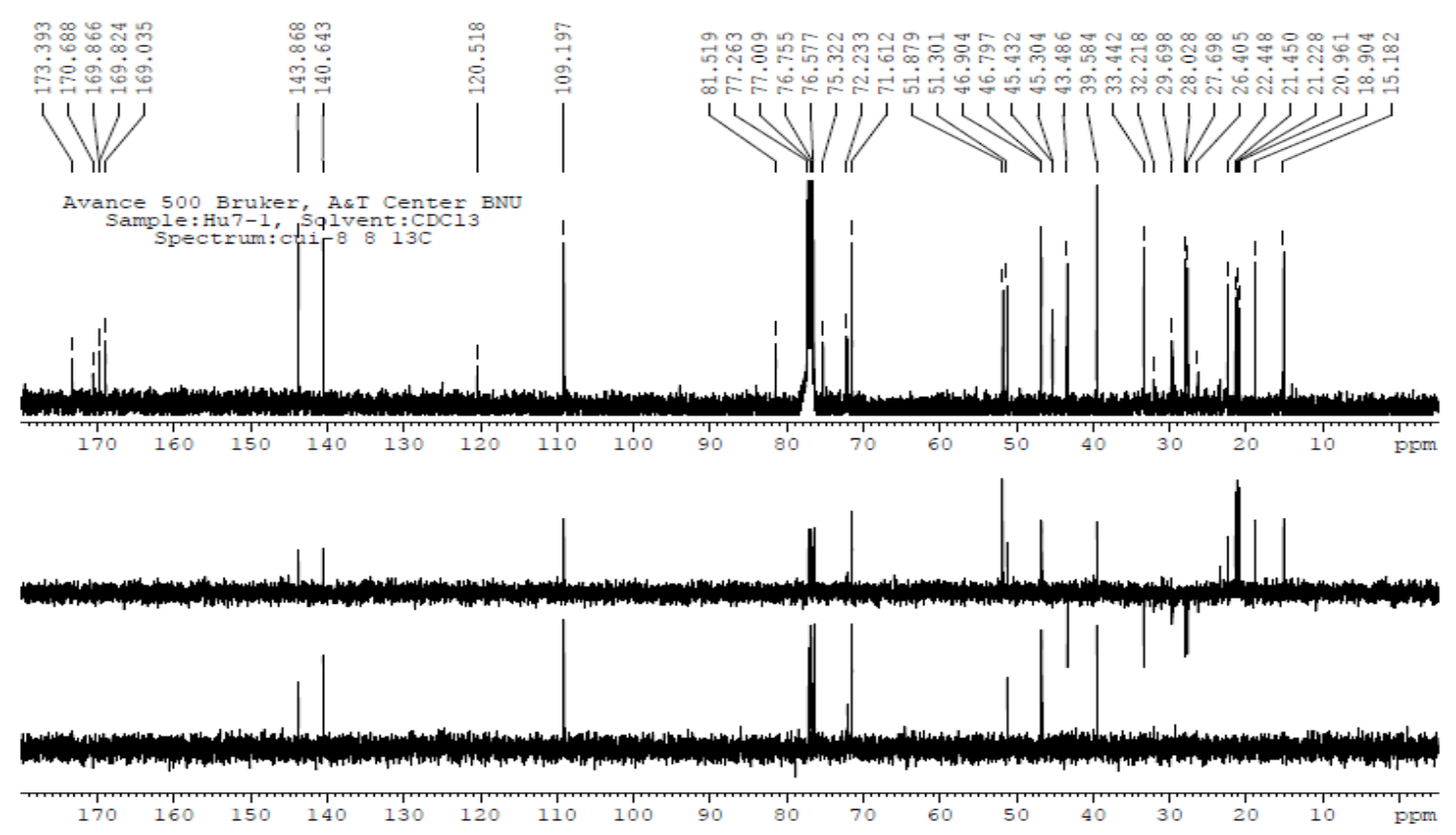

\section{HMQC spectrum}

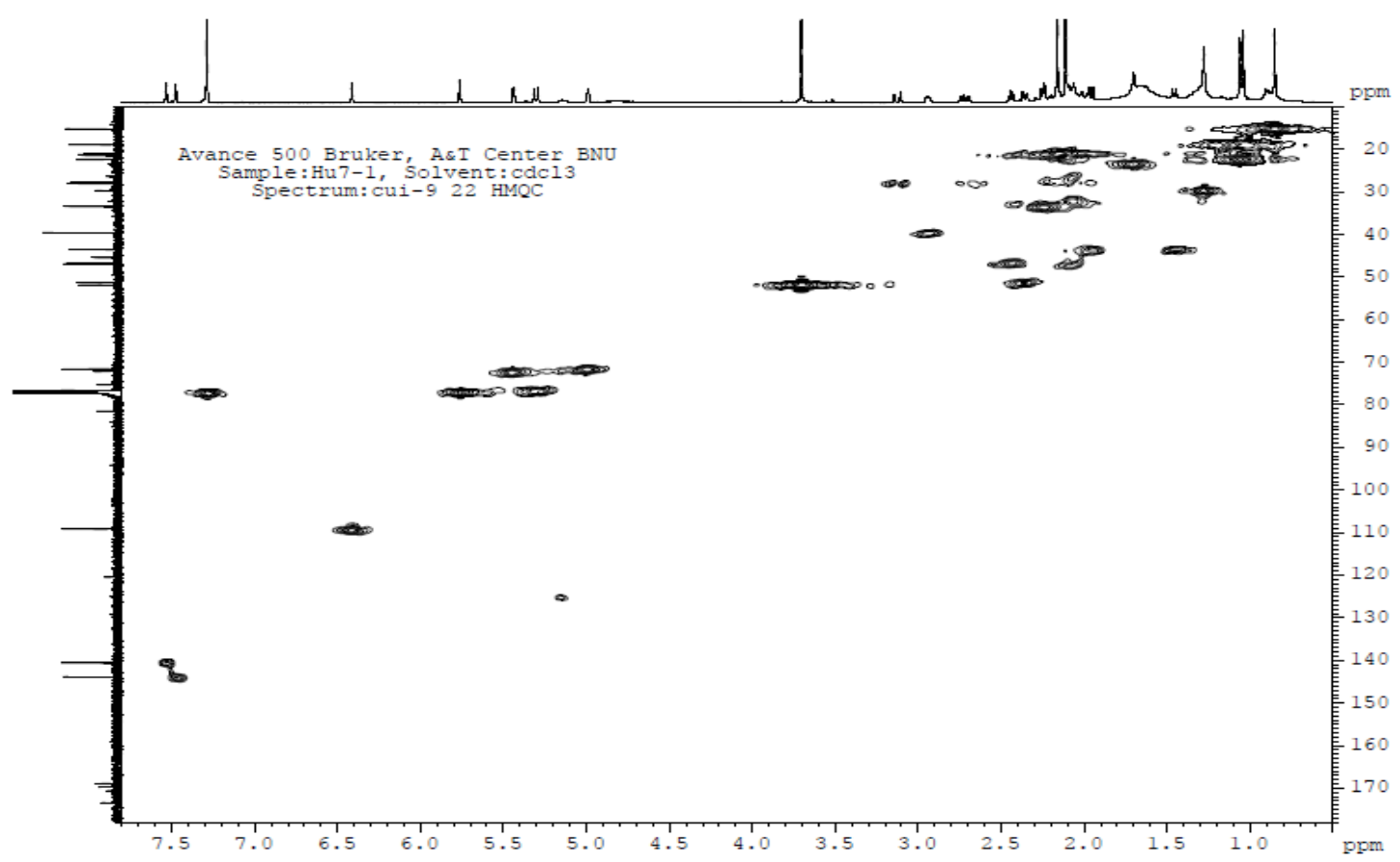




\section{HMBC spectrum}

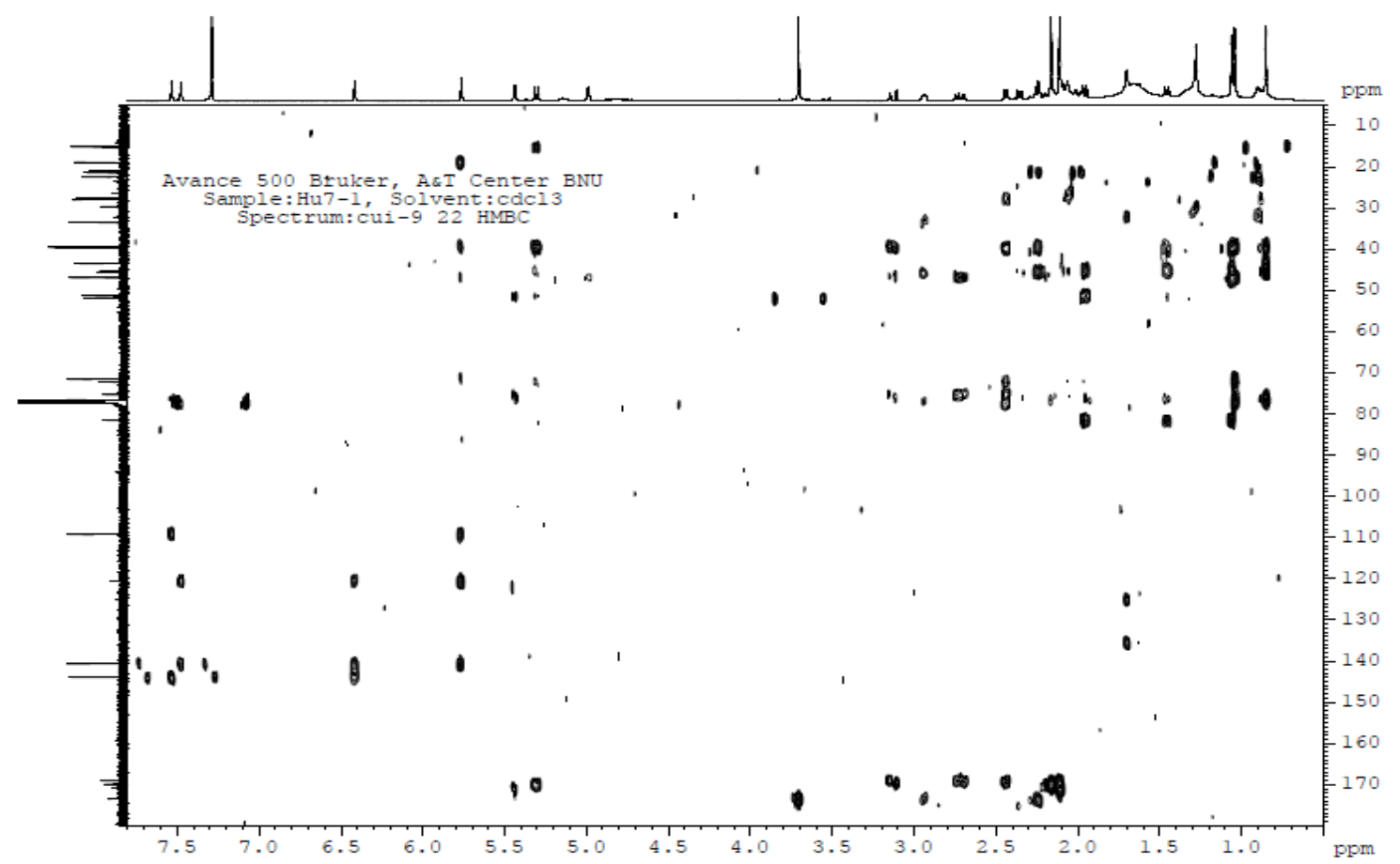

\section{NOESY spectrum}

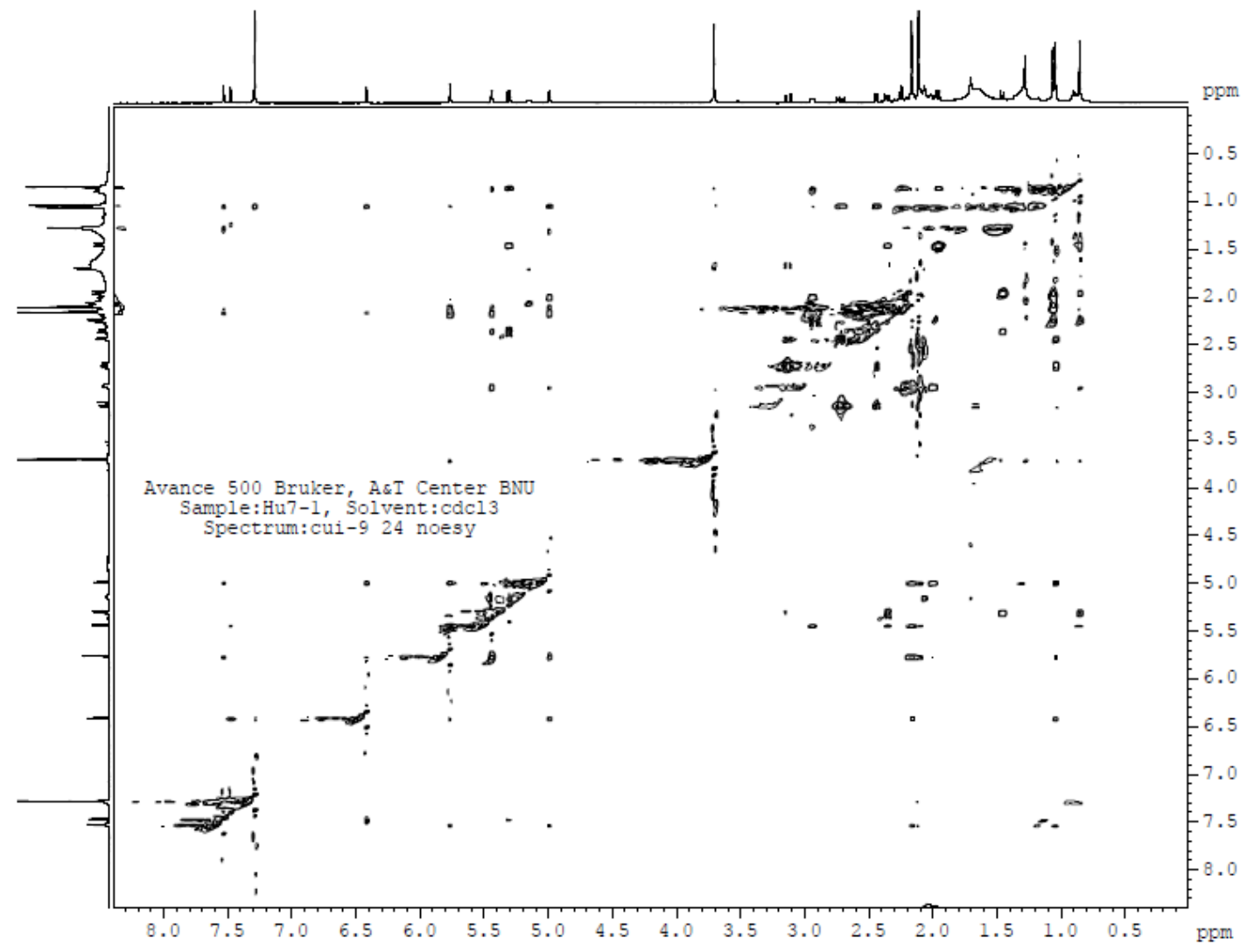




\section{ESIMS spectrum}

Workgtation: QSTAR

Princing Date: Tuesday, September 27, 2005

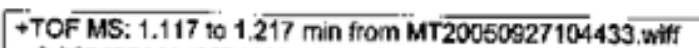
a $=3.56360714143574090 \mathrm{e}-004, \mathrm{t} 0=4.93096040019772770 \mathrm{e}+001$, subtractod ...

Max 356.8 counts

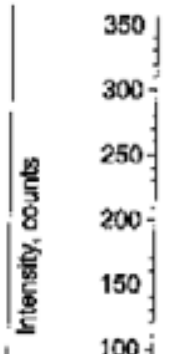

267.1399 .304 .2432

669.2083

HU7-1 ESI-TOF+

50

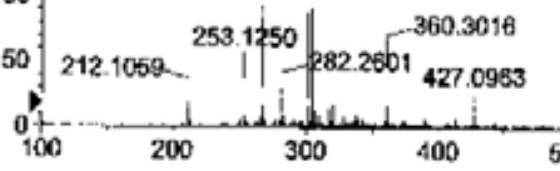

$600 \quad 600$

$m / 2$, amu

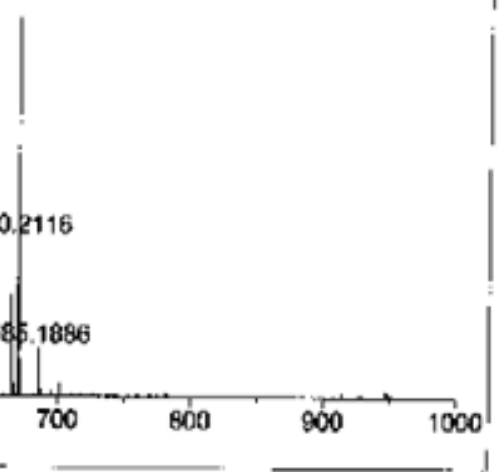

\section{HRSIMS data}

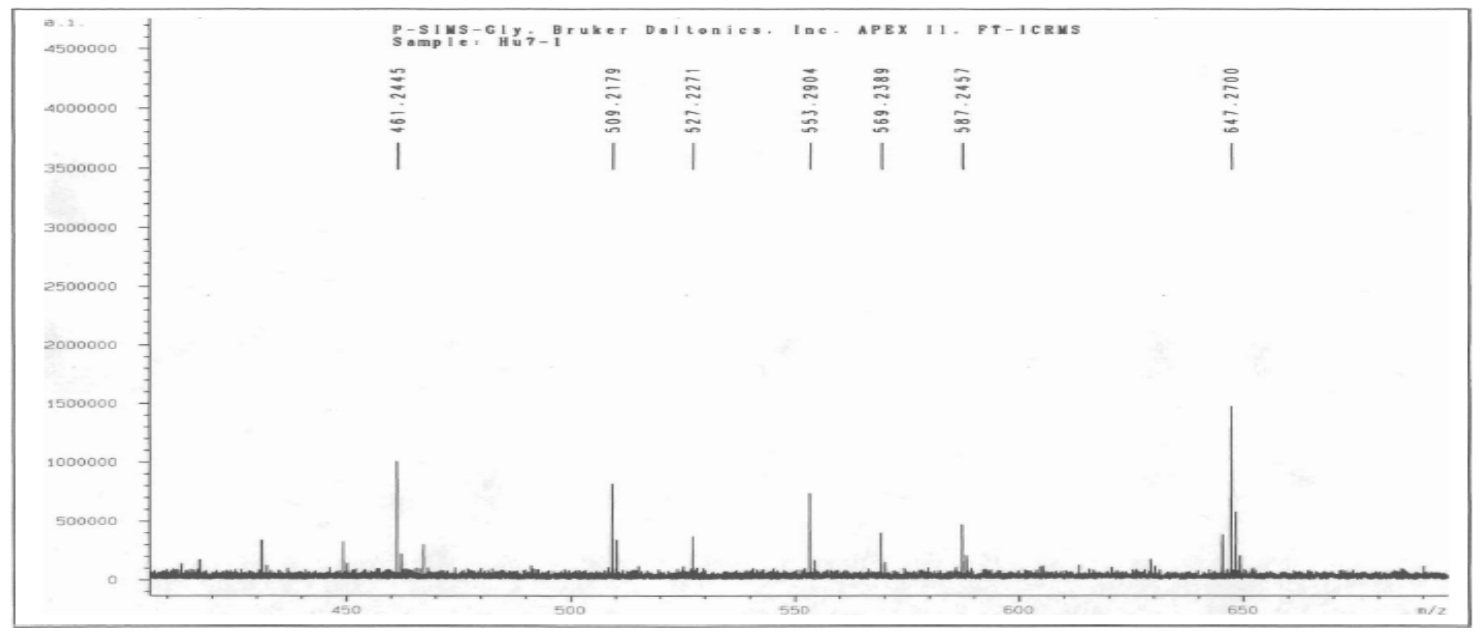

XMASS Mass Analysis for /disk2b/SPECS_SIMS/P-SIMS-WangYan/111/pdata/1/ XMASS Mass Analysis Constraints

Ion mass $=647.2699700$

Charge $=+1$

Tolerance $=0.0030000$

$\mathrm{DBE} \min =-2$

DBE $\max =200$

Max Candidates $=100$

\begin{tabular}{|c|c|c|}
\hline Atom & $\#(\min , \max )$ & Wto $(\mathrm{min}$, \\
\hline C & 30 & 100.00 \\
\hline $\mathrm{H}$ & 30 & 100.00 \\
\hline 0 & 15 & 100.0 \\
\hline
\end{tabular}

$\begin{array}{lccccc}\# & \text { C } & \text { H } & \text { O } & \text { mass } & \text { error } \\ * * * & \text { Mass } & \text { Analysis } & \text { for } & \text { mass } 647.2699700 & \\ 1 & 33 & 43 & 13 & 647.2697925 & 2.742 e-07\end{array}$ 


\section{Xylocarpin D (4)}

IR spectrum

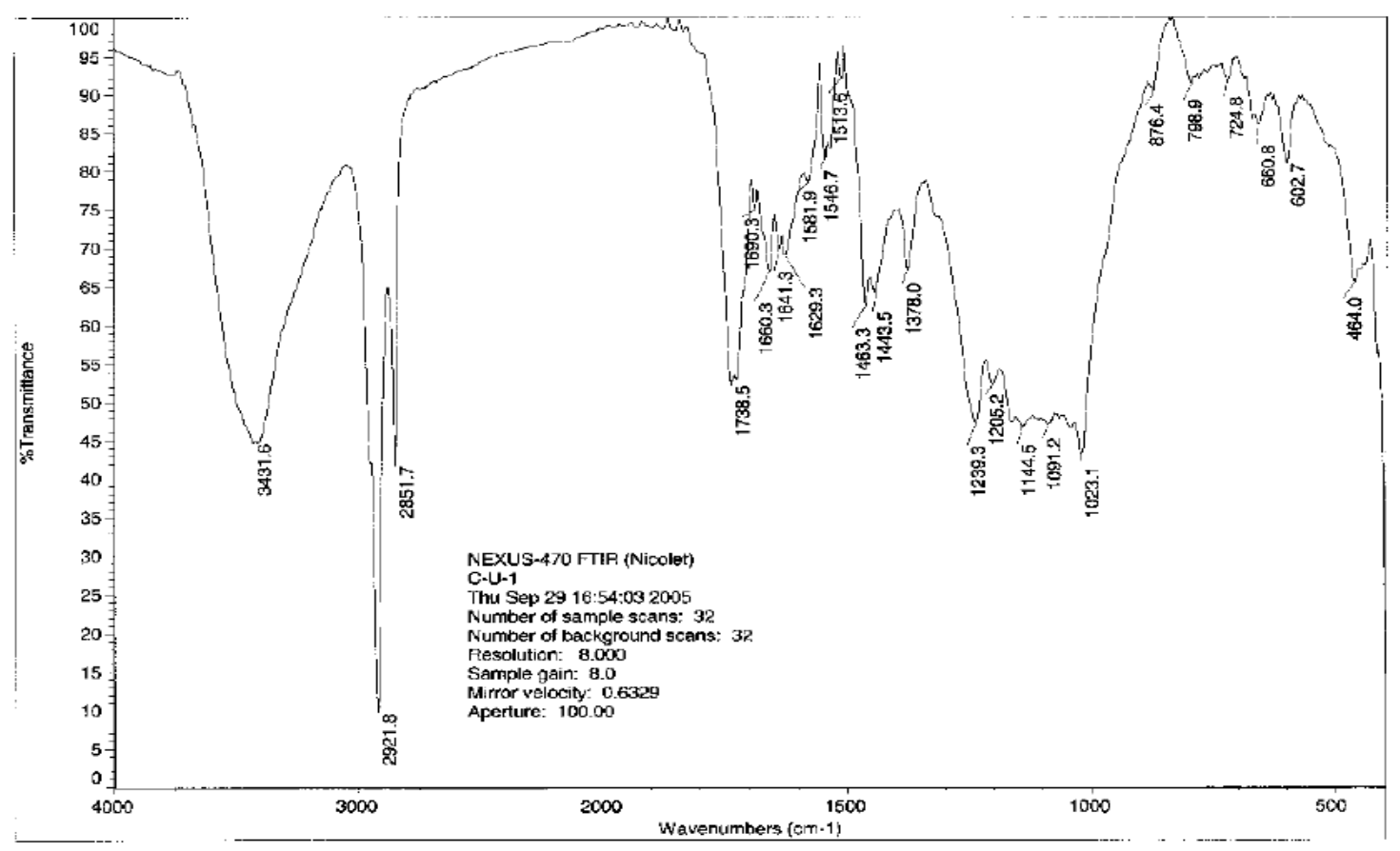

\section{${ }^{1}$ H NMR spectrum}

Avance 500 Bruker, AET Center BNU
samp 1e: $c-\mathbf{u}-1$. Solvent: dnso

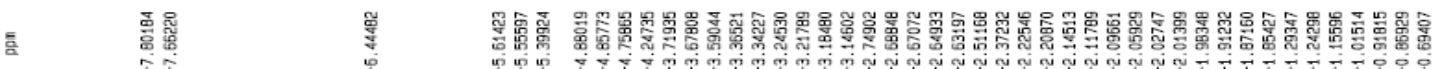
11
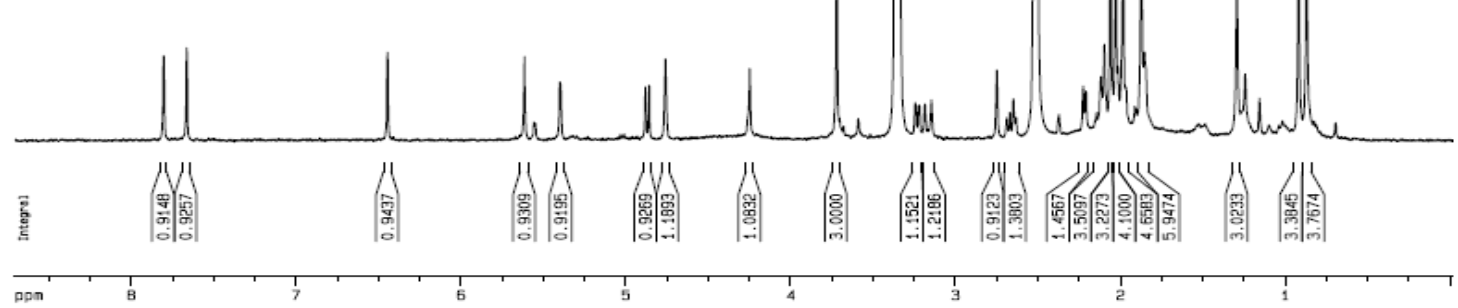


\section{${ }^{13} \mathrm{C}$ and DEPT NMR spectra}
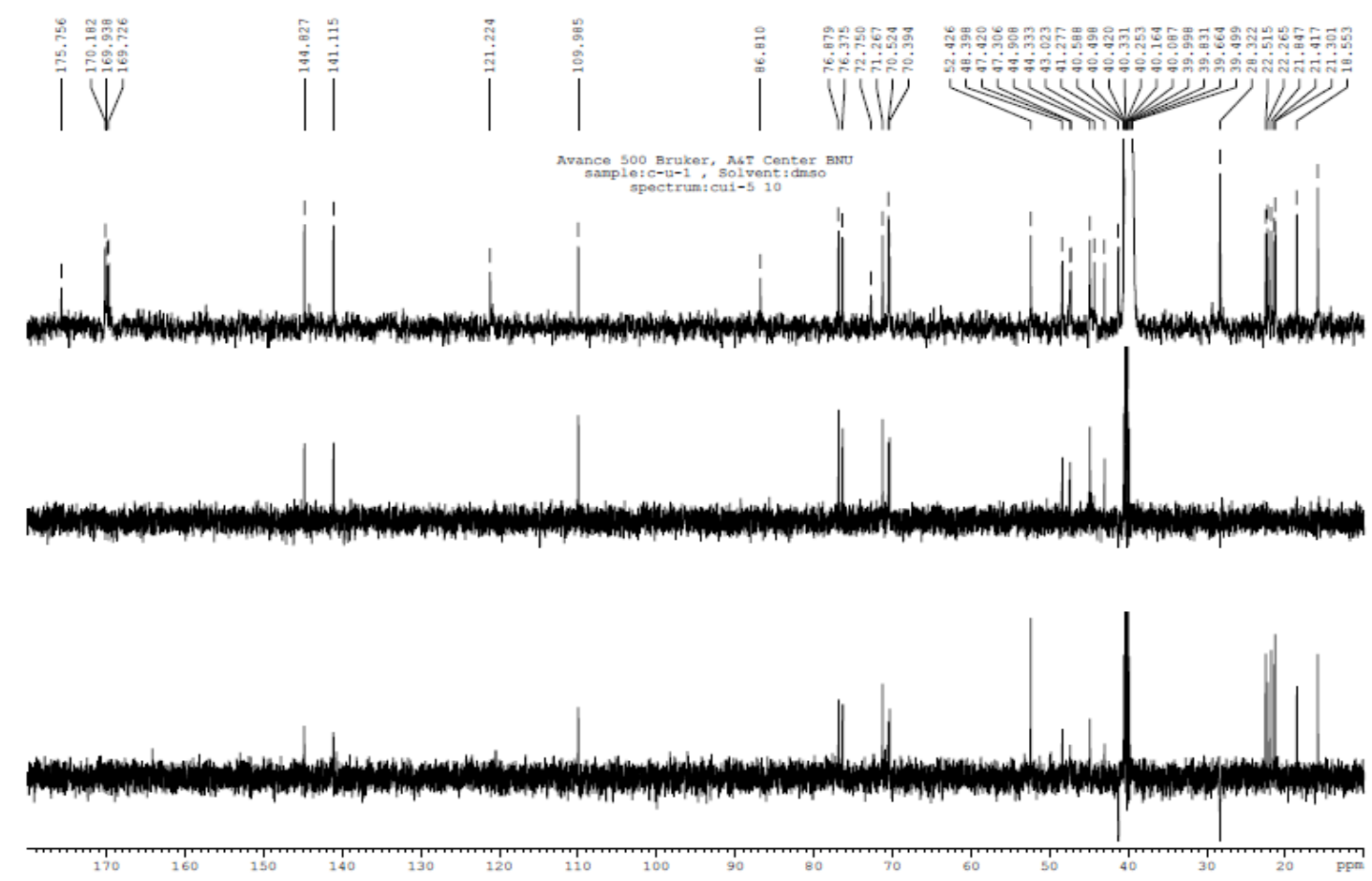

\section{HMQC spectrum}

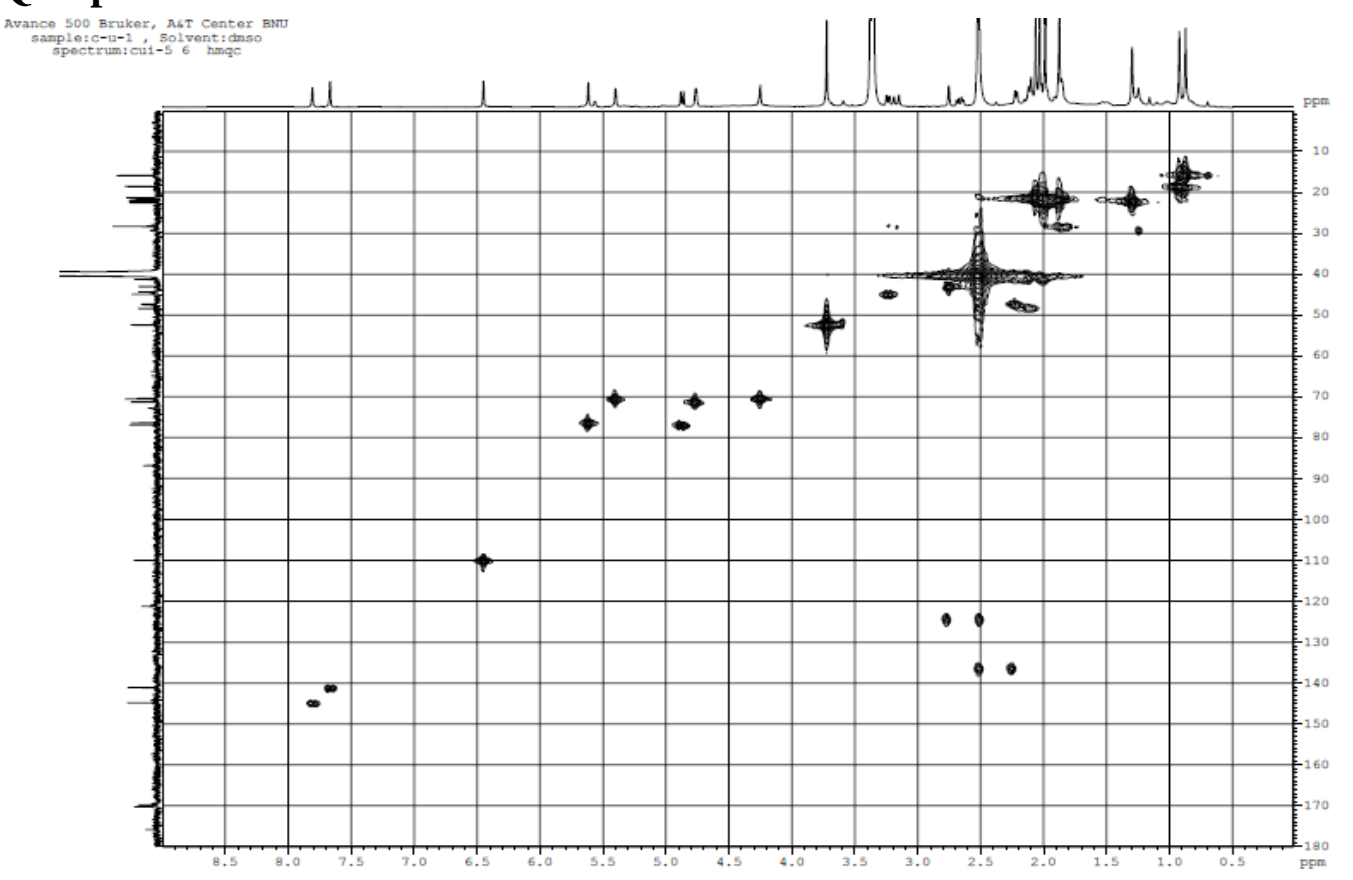




\section{HMBC spectrum}

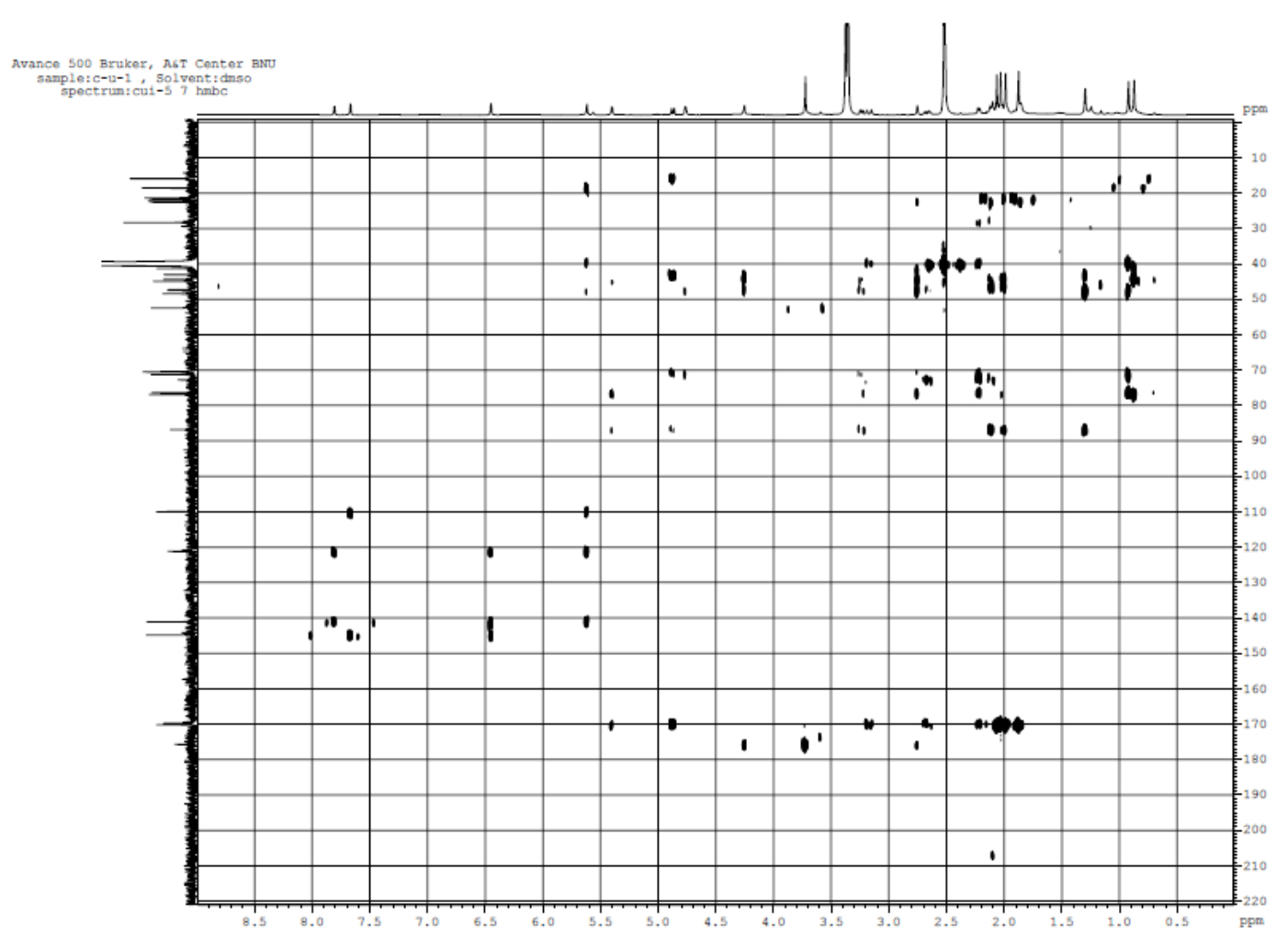

\section{DQFCOSY spectrum}

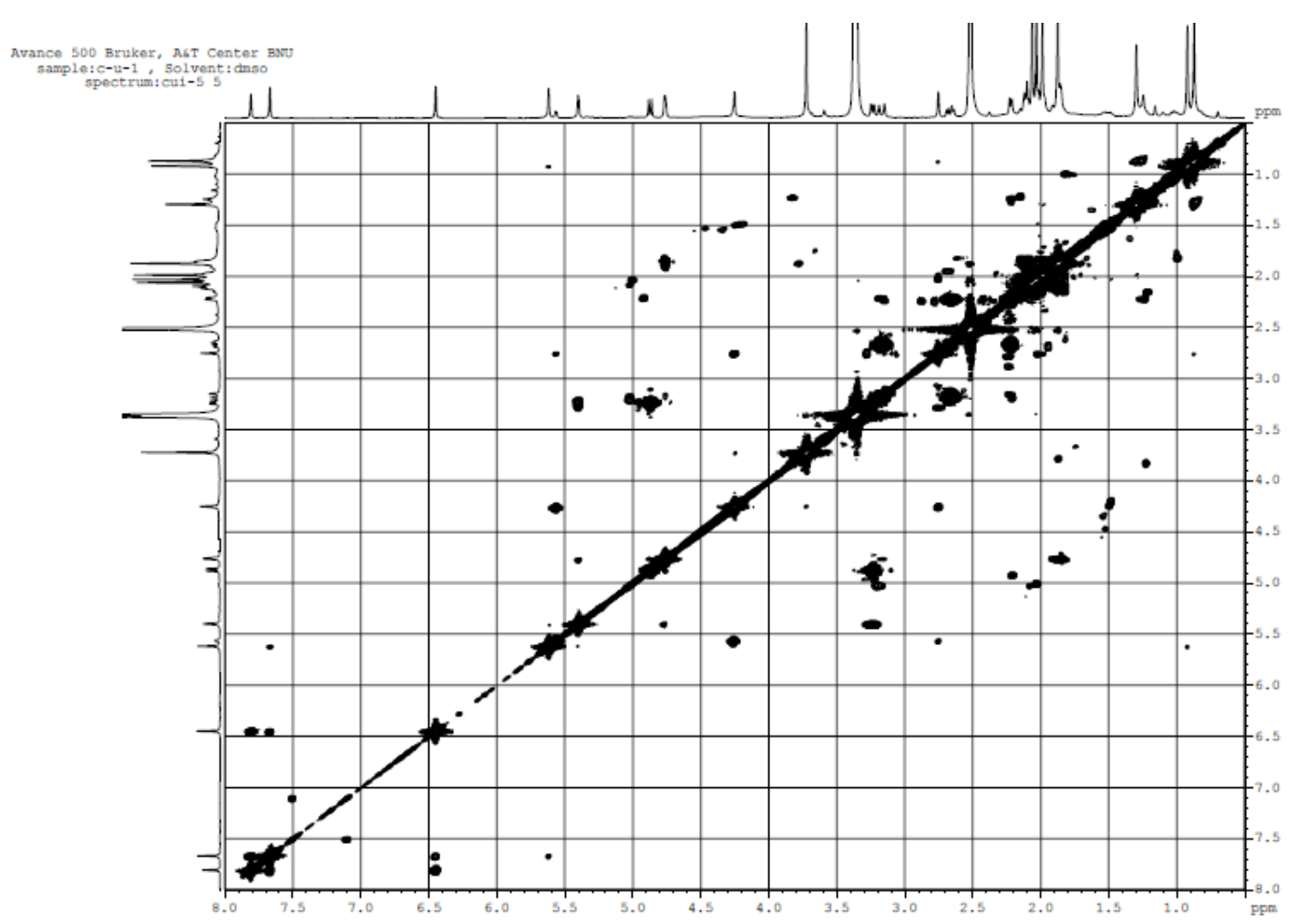




\section{ESIMS spectrum}

wOrkBtation: QSTAR

Printing Date: Monday. July 11, 2005

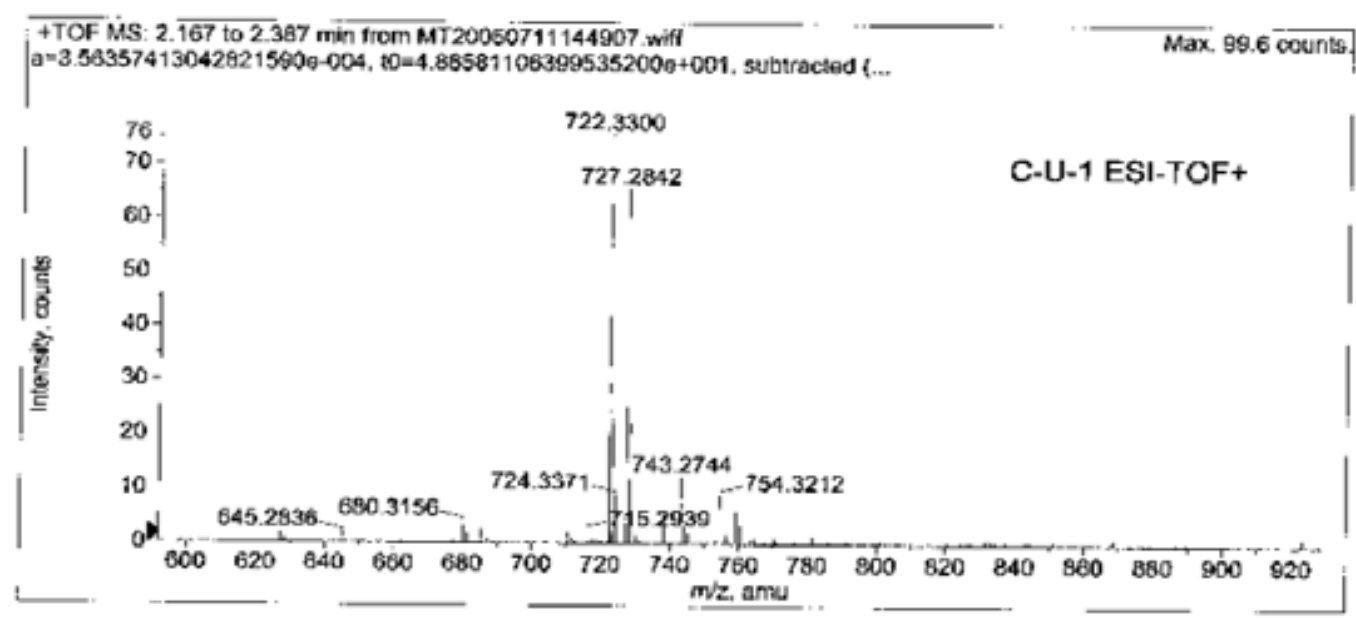

\section{HRSIMS data}

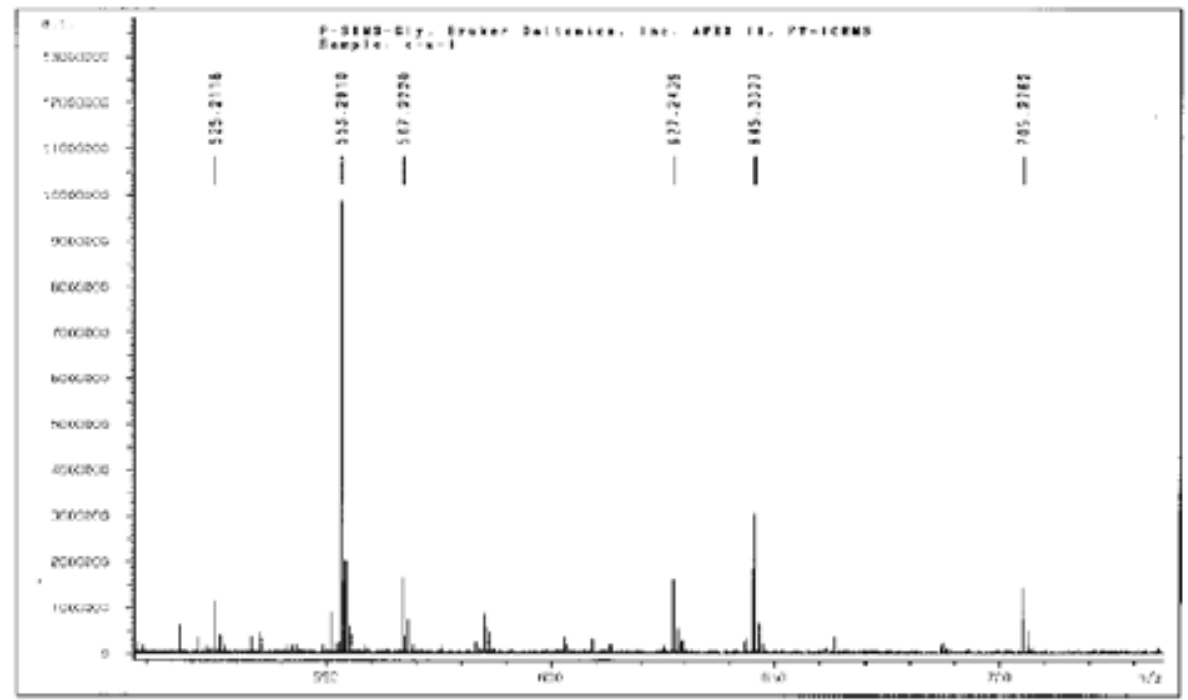

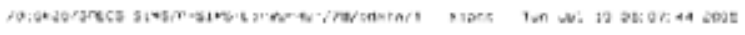

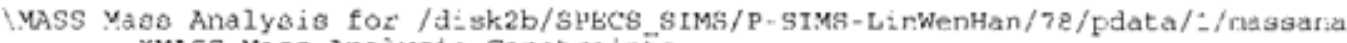
XMAsS Msss Anaiyais Co:1atrainta

Ion thas $=705.276162 \mathrm{C}$

Charge $=+1$

Tolerance $-0,0030000$

DAF nin - - 7

LUE $\max -20 \mathrm{C}$

Max Candidatea - 100

$\begin{array}{lllll}\text { Acom } & \#\langle\operatorname{nin}, \max \rangle & \text { We }\{\langle\min , \max \rangle \\ \mathrm{C} & 30 & 40 & 0.00 & 100.00 \\ \mathrm{E} & 30 & 70 & 0.00 & 200.00 \\ 0 & 1 \mathrm{C} & 20 & 0.00 & 100.00\end{array}$

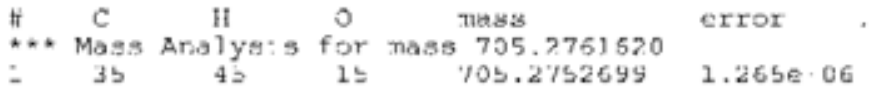




\section{Xylocarpin E (5)}

IR spectrum

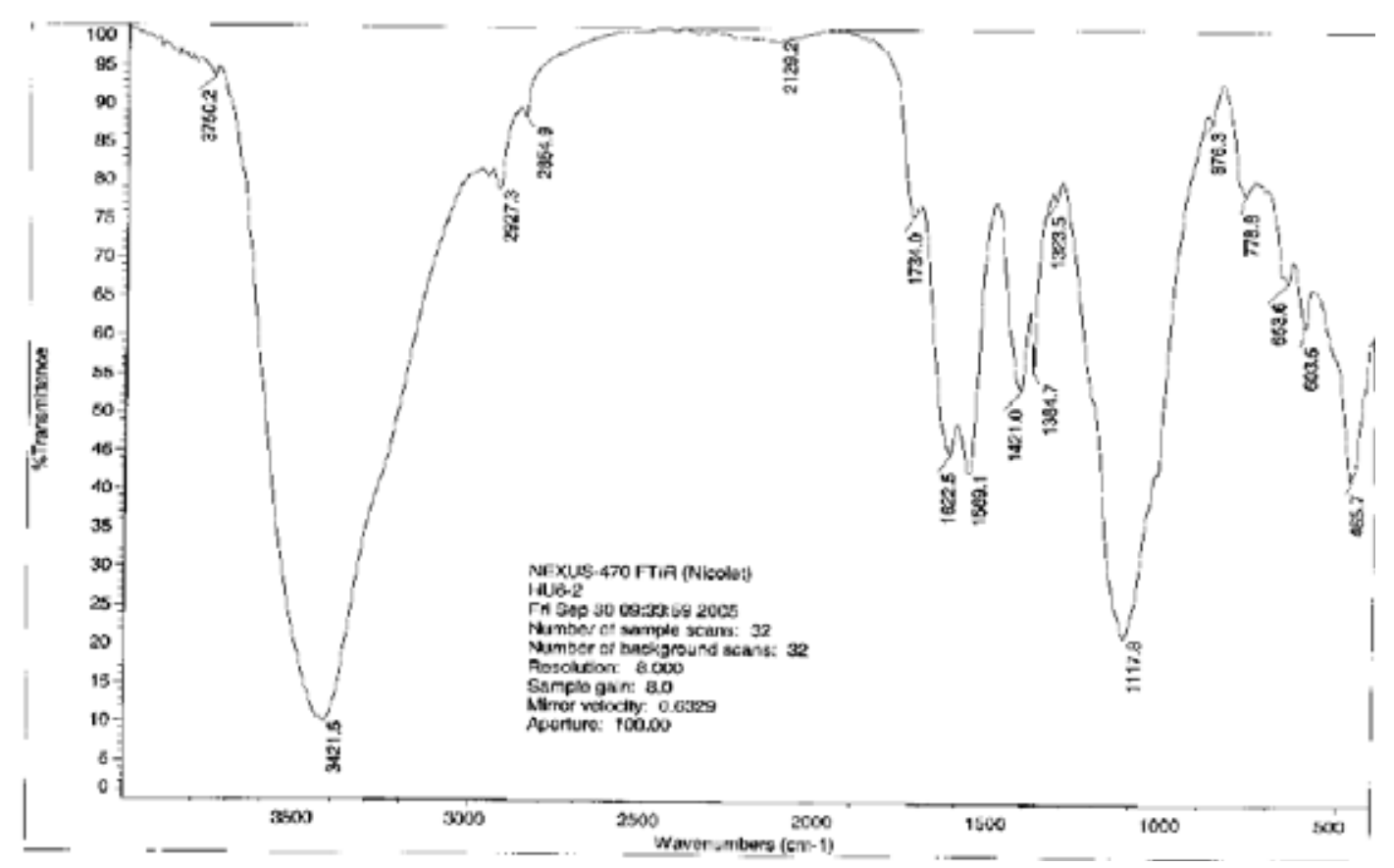

\section{${ }^{1} H$ NMR spectrum}

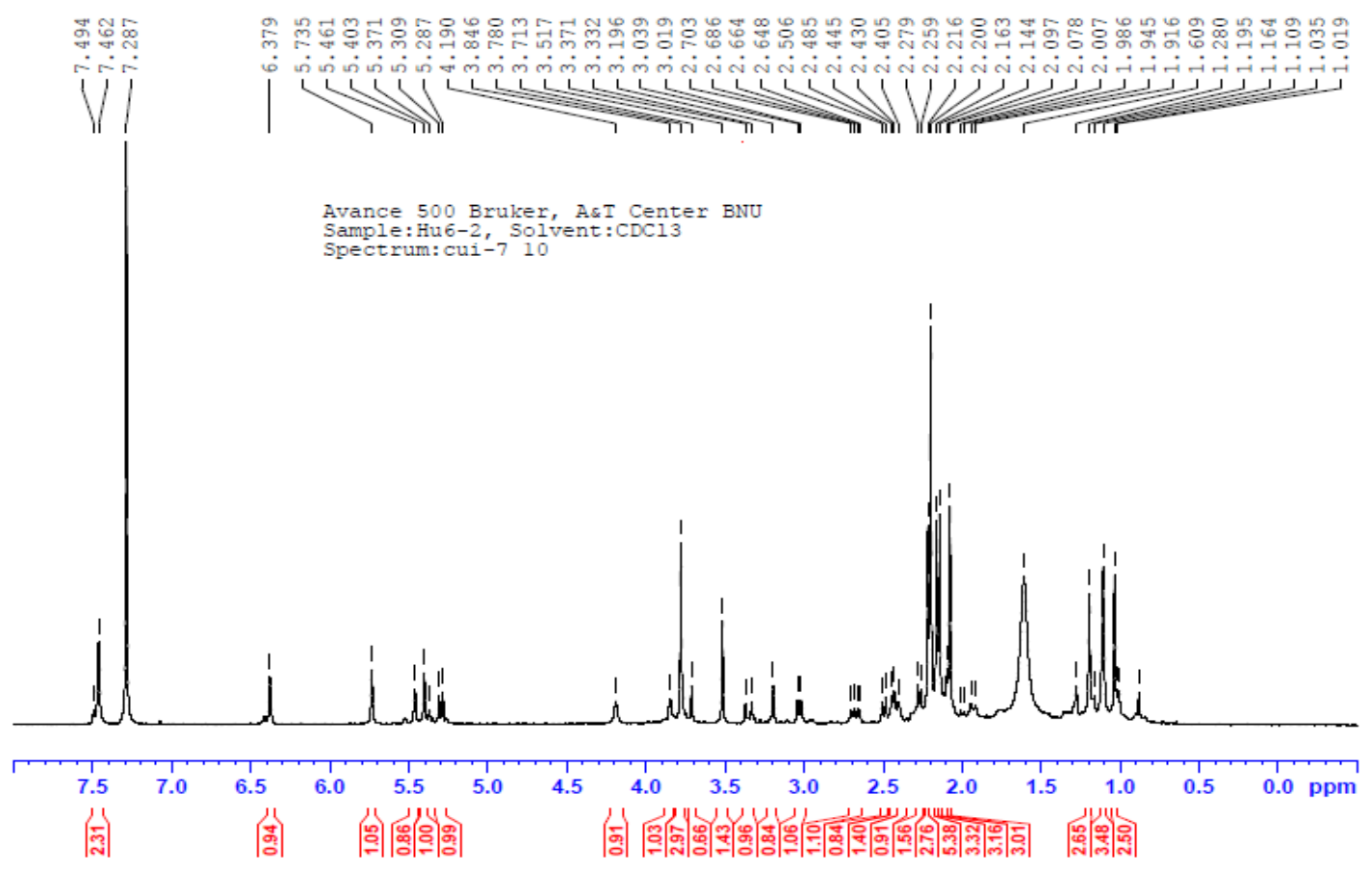




\section{${ }^{13} \mathrm{C}$ NMR spectrum}

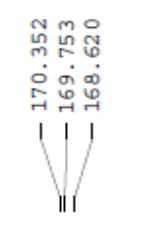

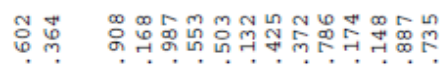

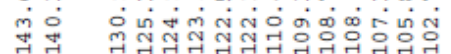

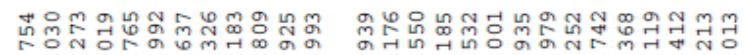

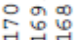

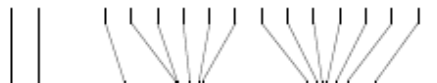

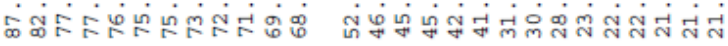

Avance 500 Bruker, A\&T Center BNU

sample:Hu6-2, Solvent: $\mathrm{CDCl}_{3}$

spectrum: cui-7 17

I IIIIIII III II III

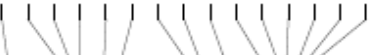

11il

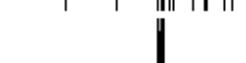

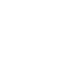




\section{HMBC spectrum}

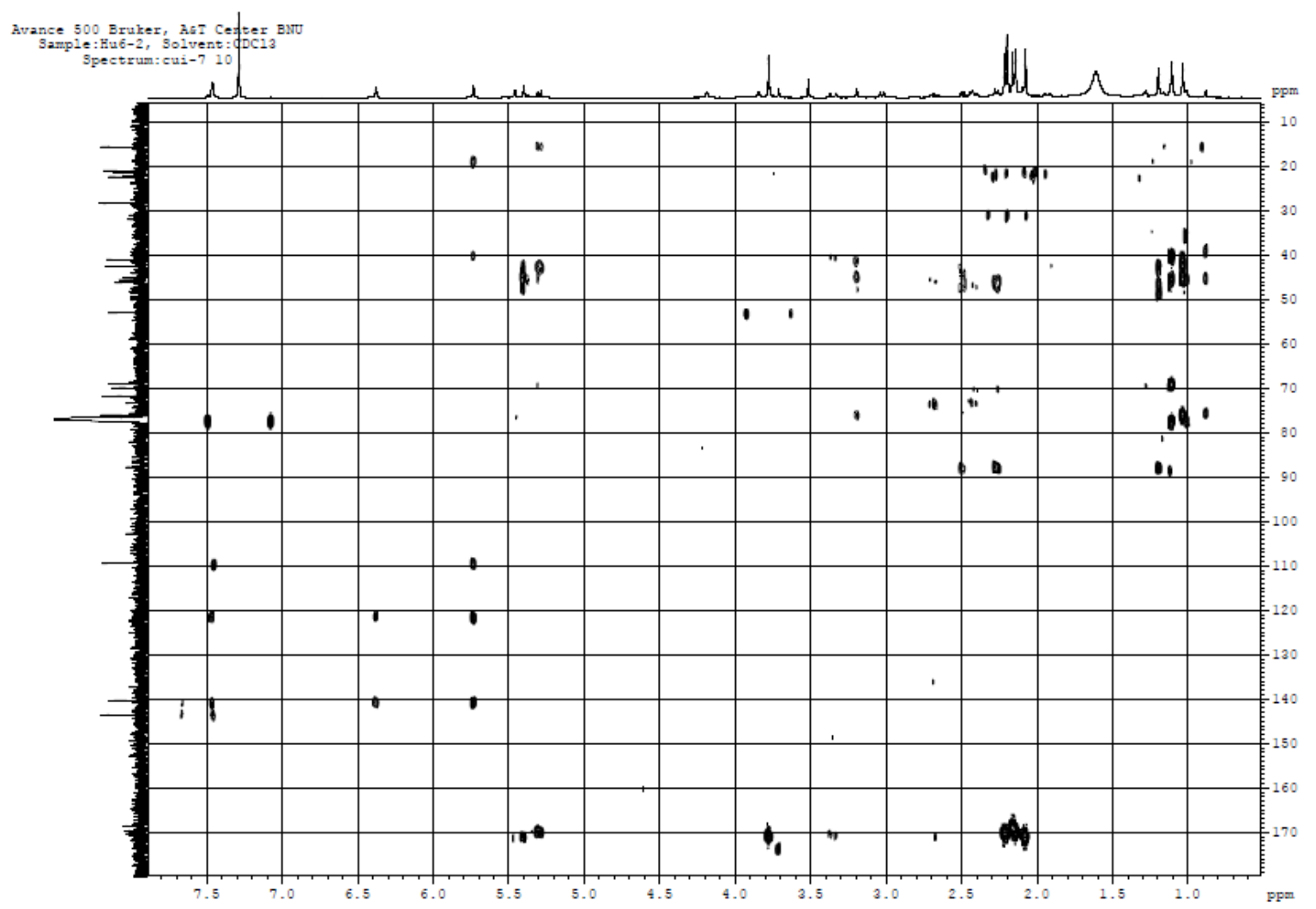

\section{DQFCOSY}

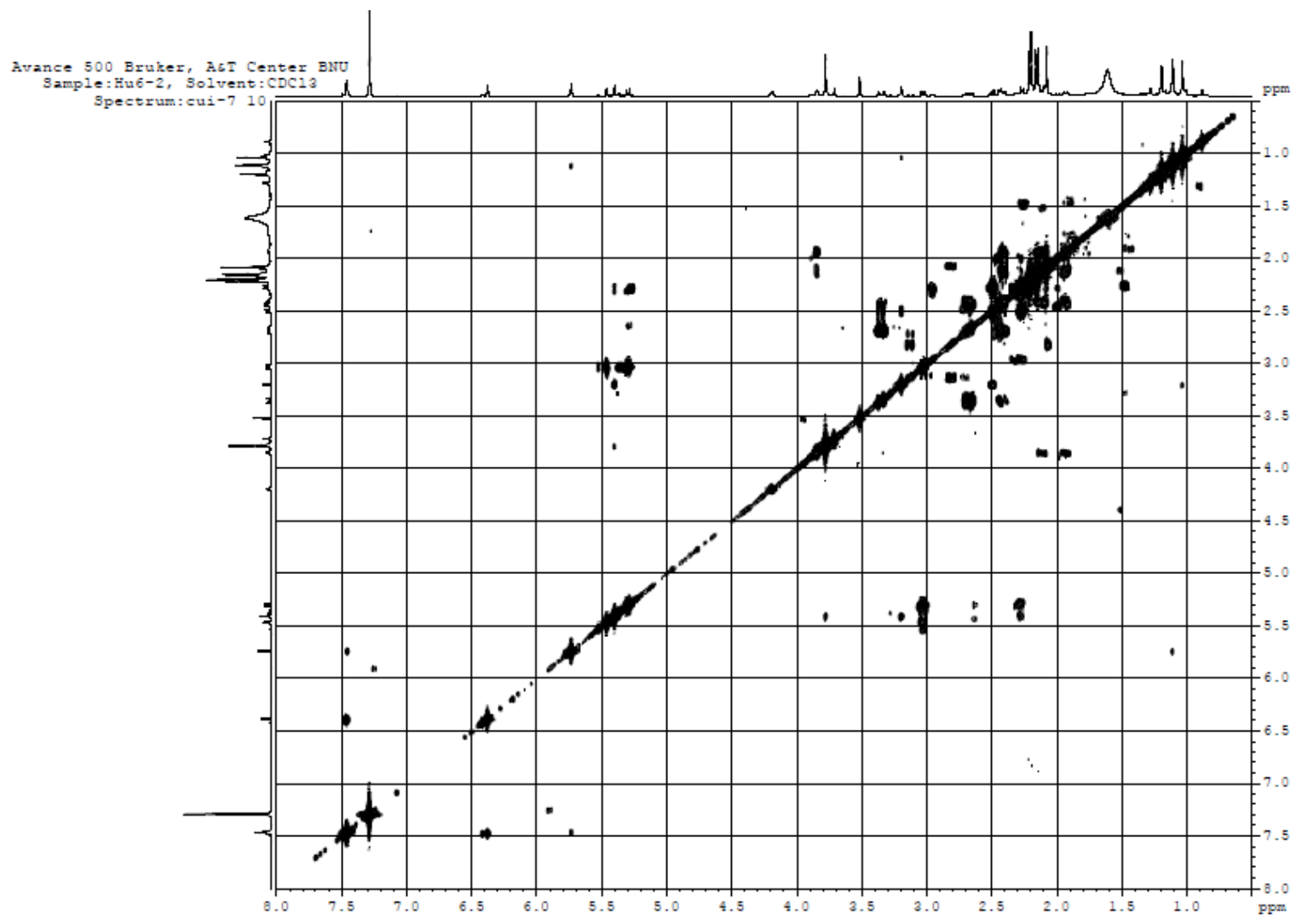




\section{ESIMS spectrum}

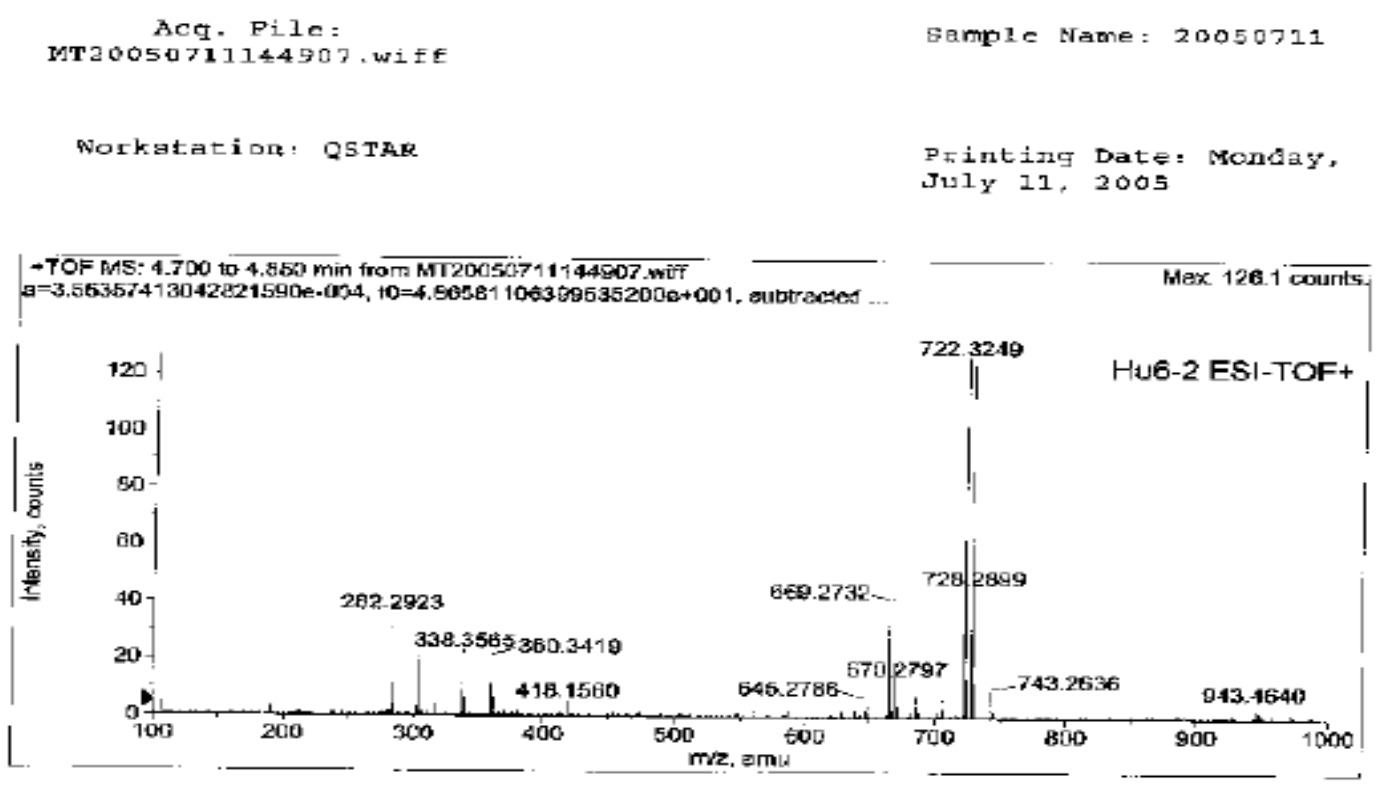

\section{HRSIMS data}

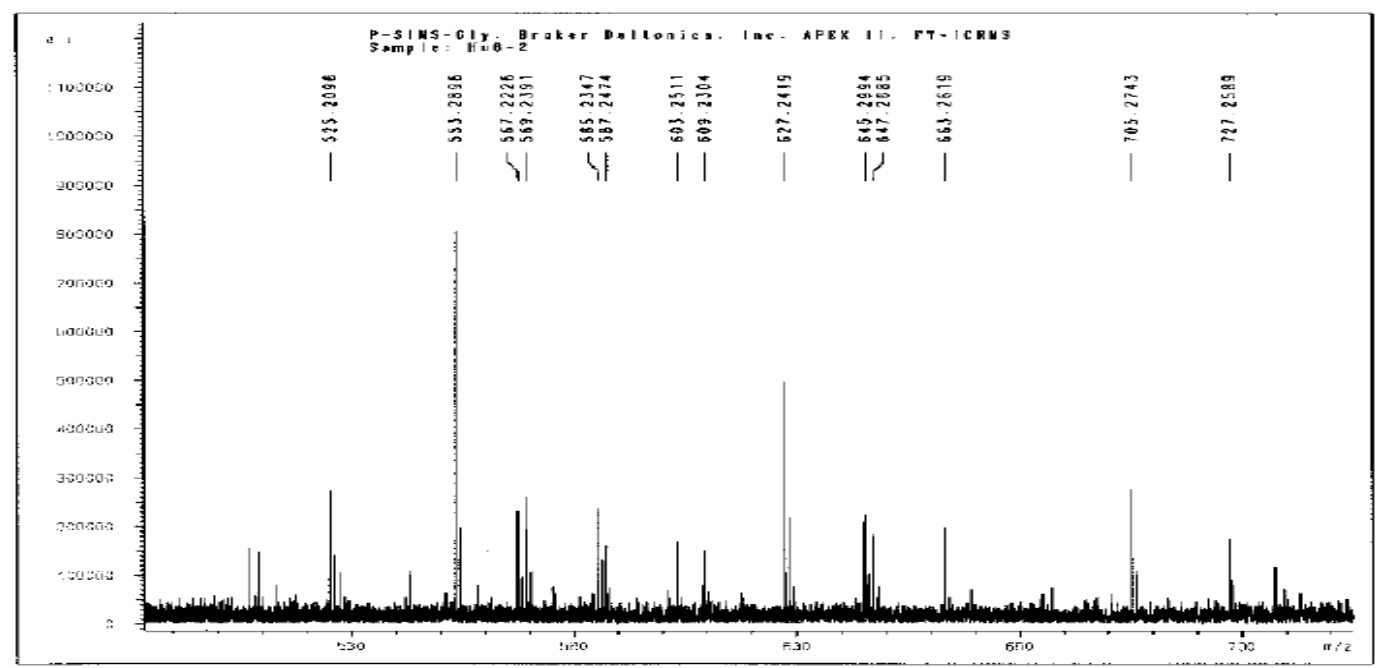

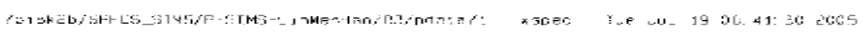

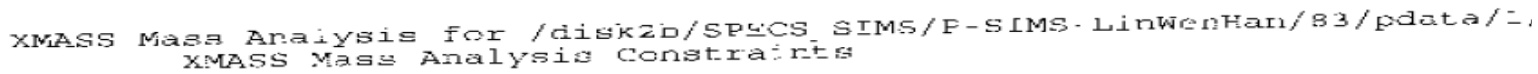

Ior: rass = 705.2743130

Charge $=+1$
Tclerance $=0.0050000$

$\begin{array}{ll}\text { DBE } \min = & -2 \\ \text { DEF } \max = & 200\end{array}$

Nax Cariabaies - 100

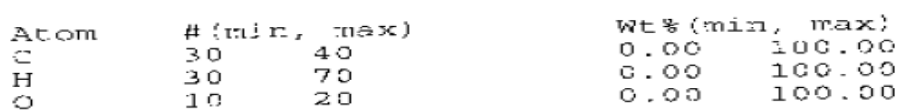

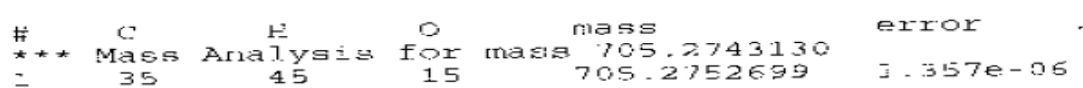




\section{6-dehydroxyxylocarpin D (6)}

\section{IR spectrum}

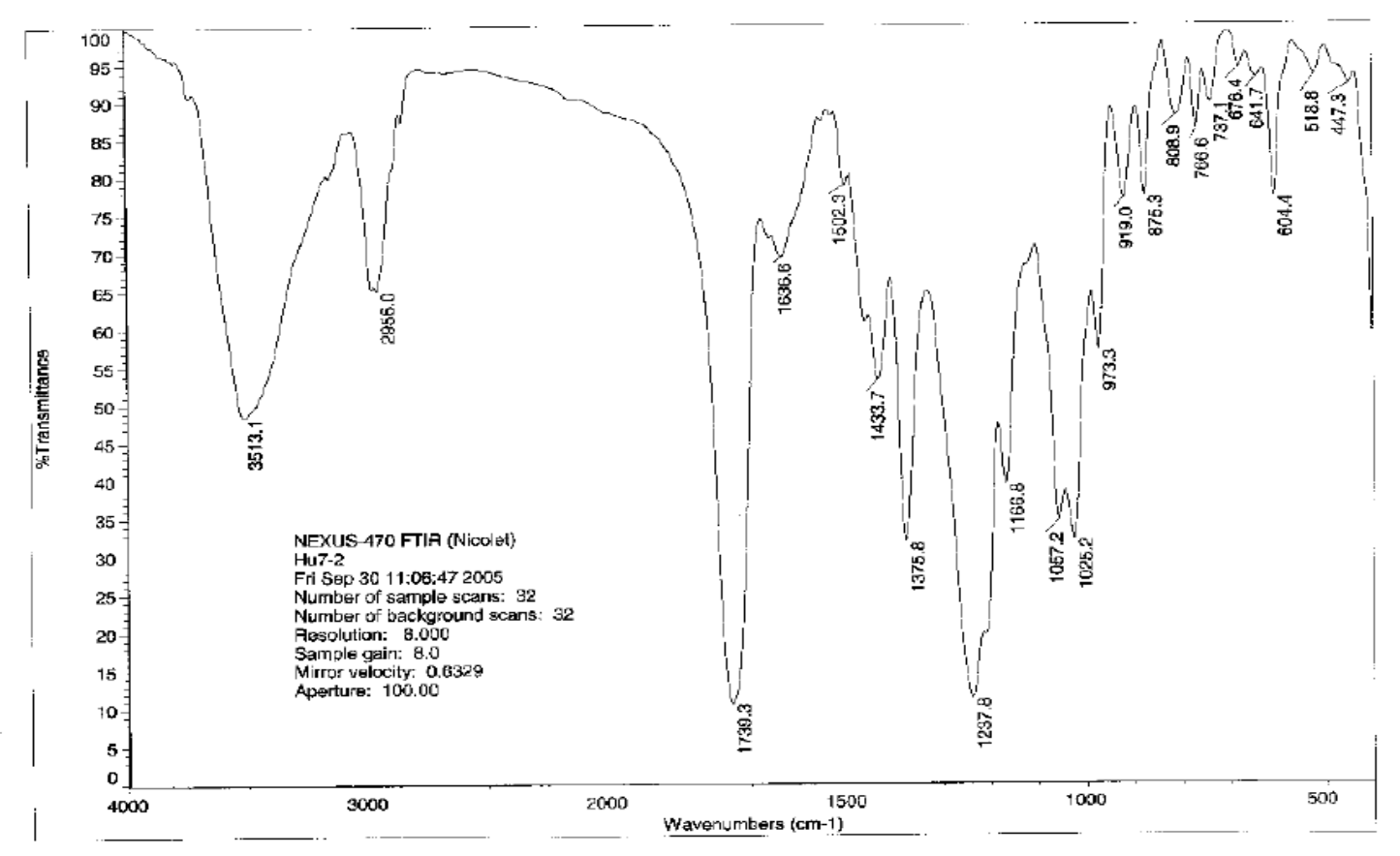

\section{${ }^{1}$ H NMR spectrum}

Avance 500 Bruker. A\&T Center BNU mole: Ha7-2 Solvent: cac13

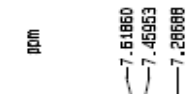

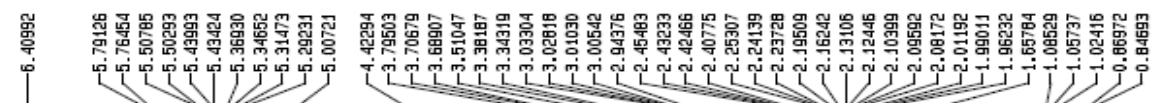
1 lill

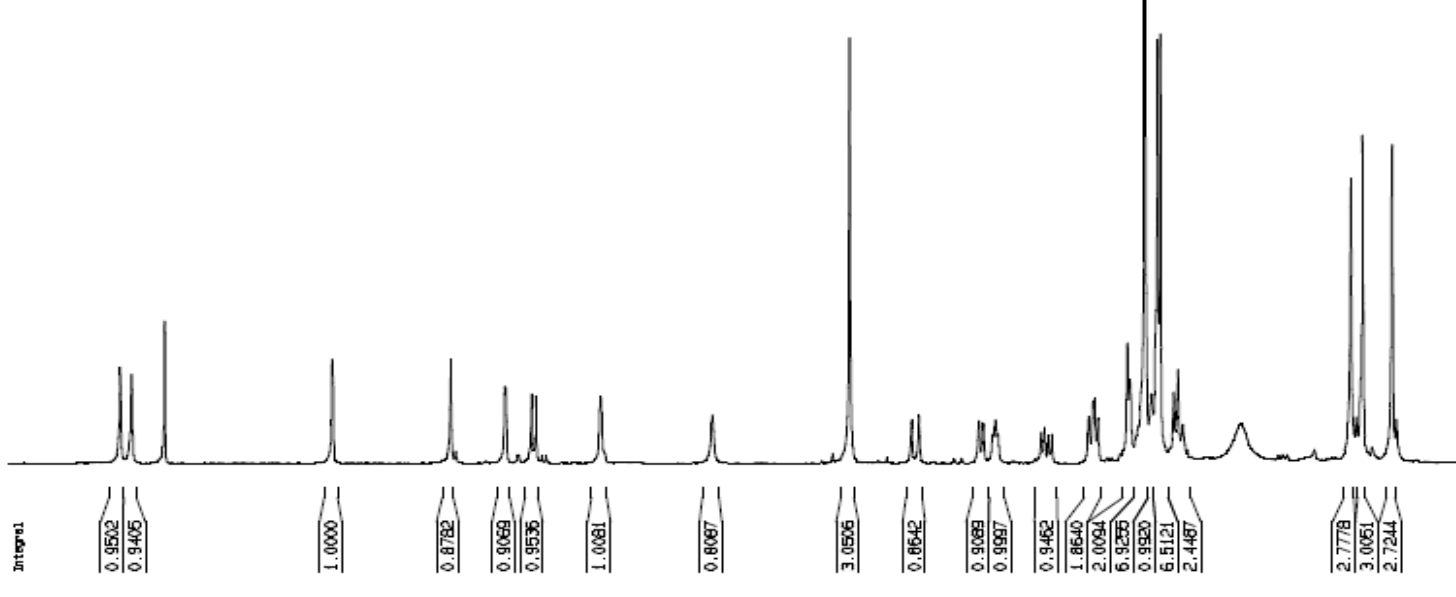

ppon 


\section{${ }^{13} \mathrm{C}$ and DEPT NMR spectra}

Avance 500 Bruker, AST Center BNU Sample: :N7-2 Solvent: Cdc13 $^{-13}$
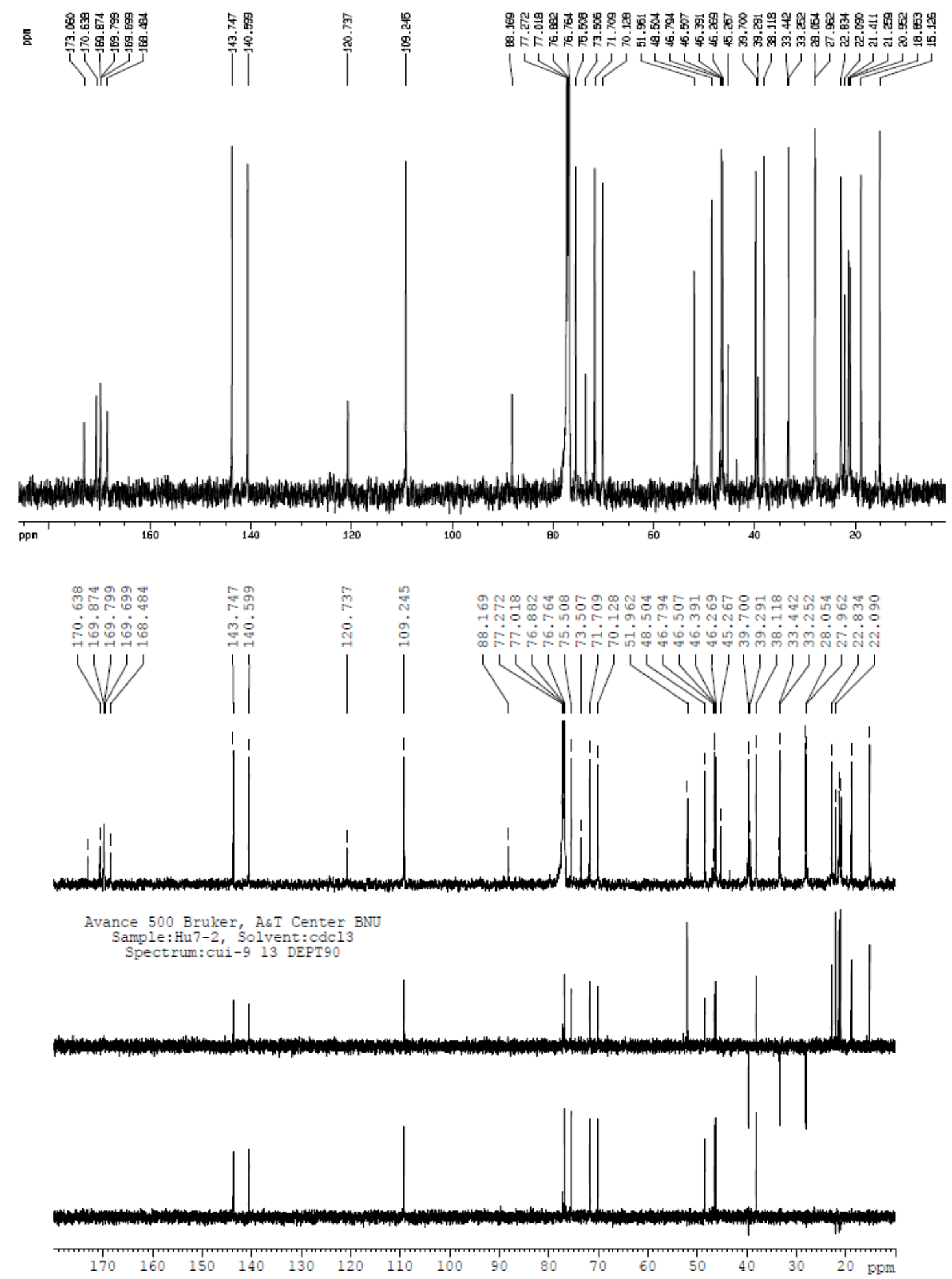


\section{HMQC spectrum}

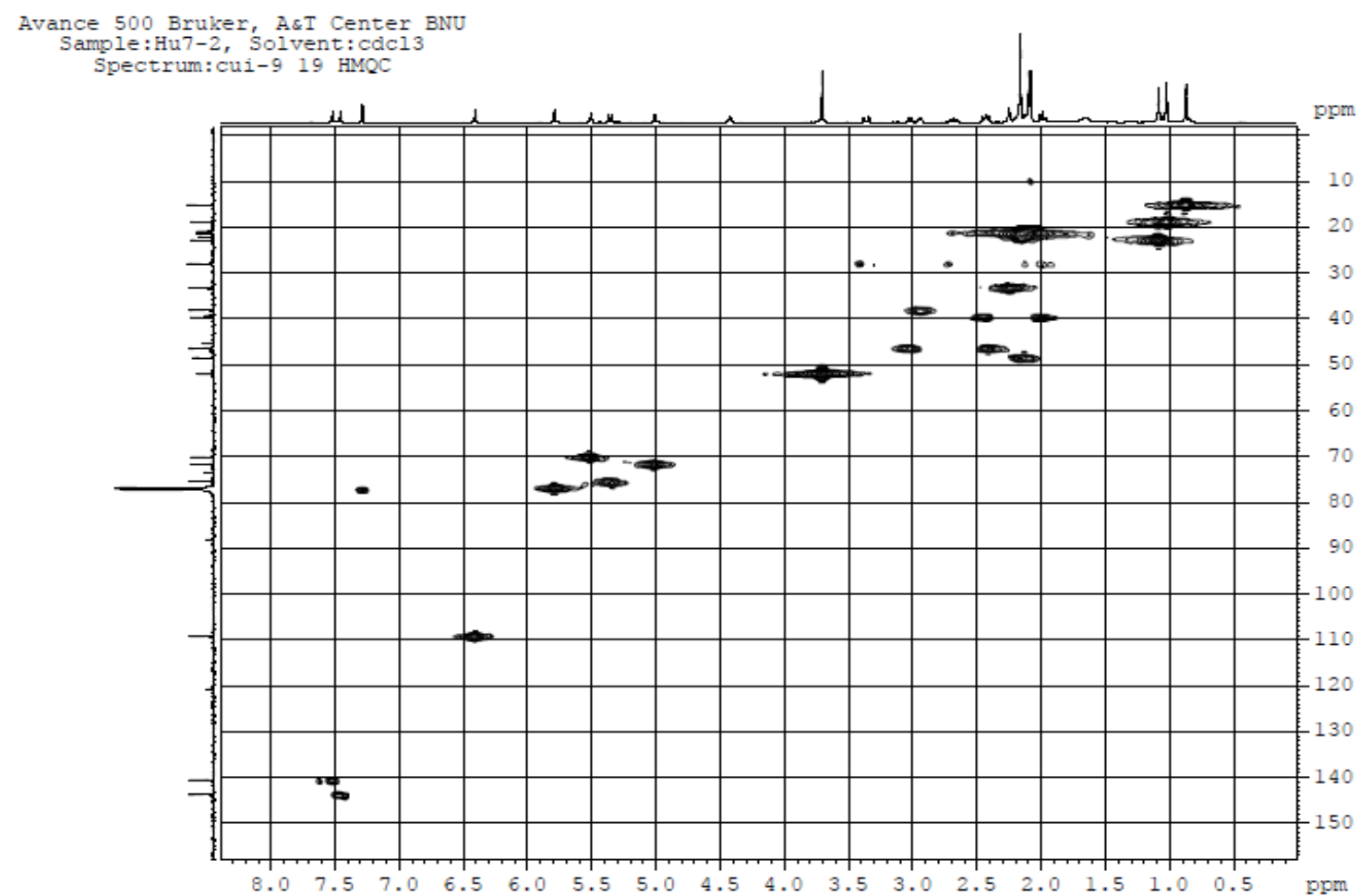

\section{HMBC spectrum}

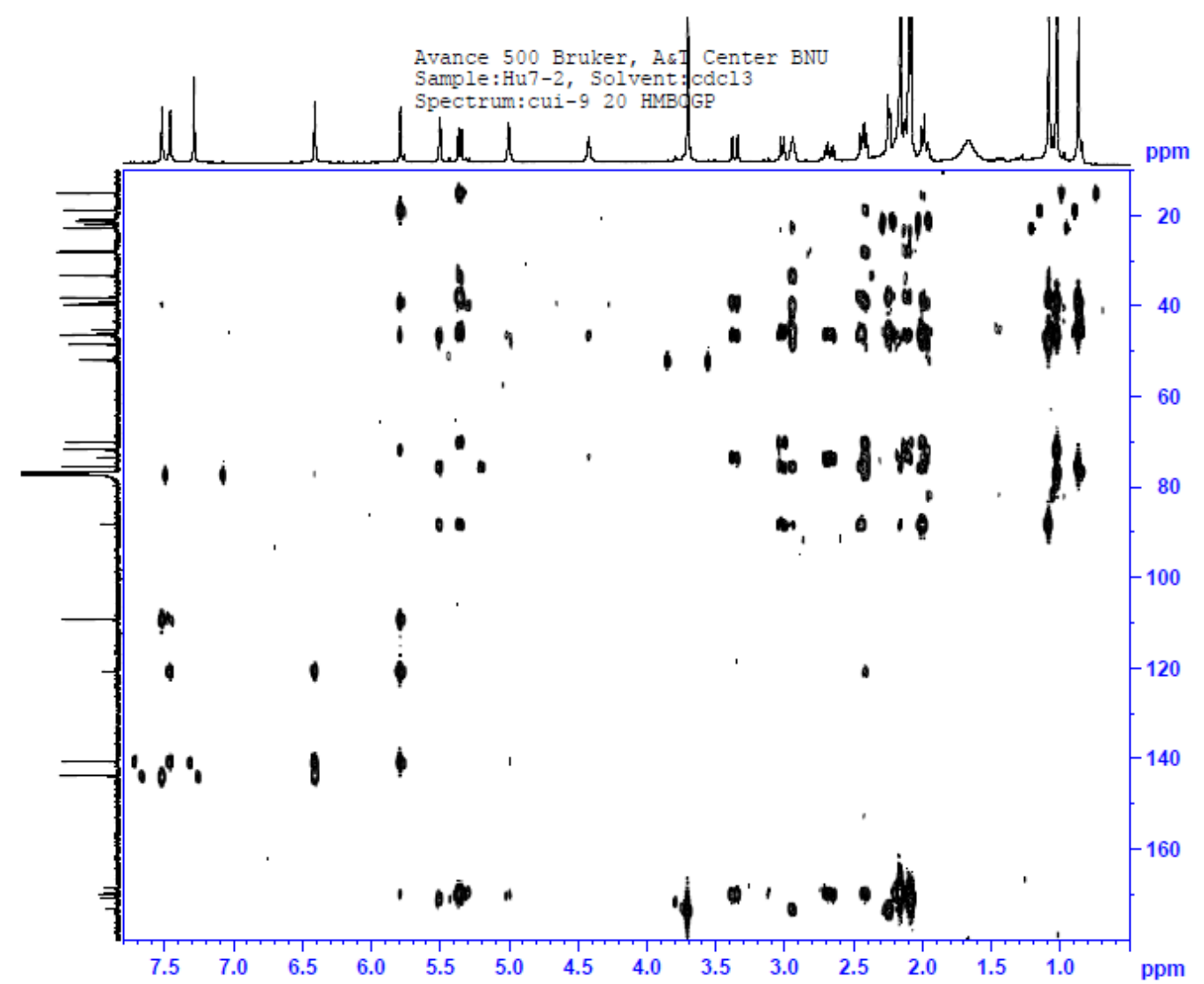




\section{NOESY spectrum}

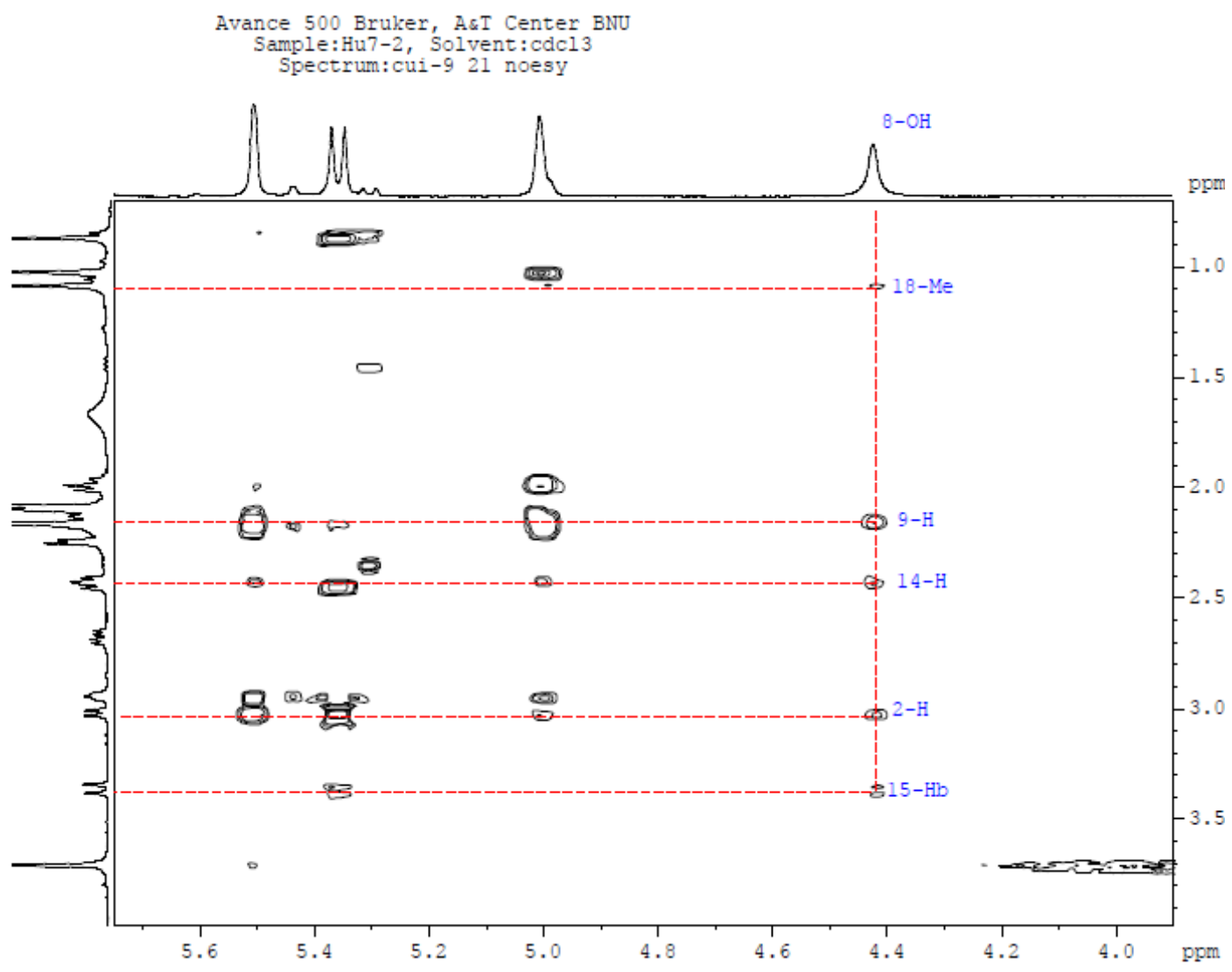

\section{ESIMS spectrum}

Acq. File: MT20050927104433.wiff

Sample Name:

Workstation: QSTAR

Frinting Date: Tuesday, September 27,2005

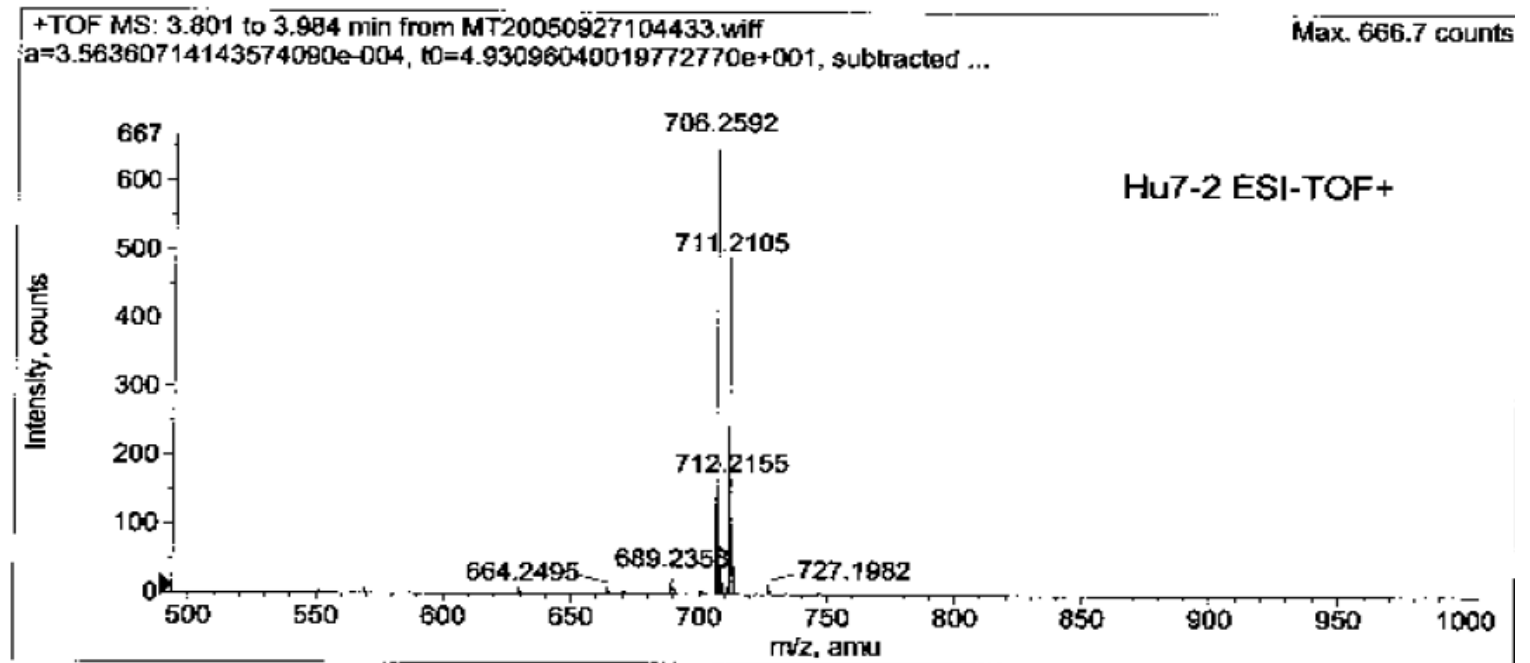




\section{HRSIMS data}

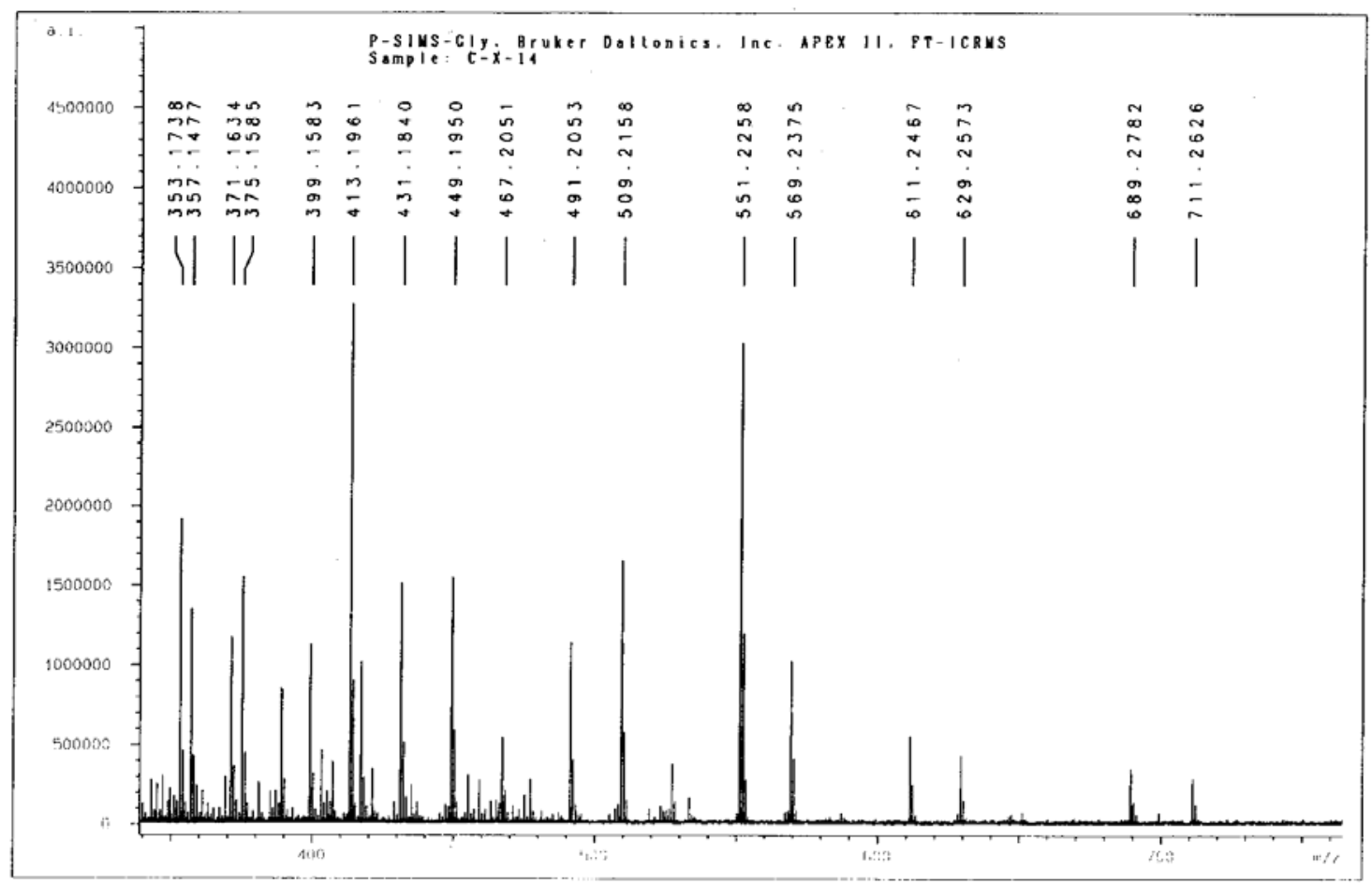

XMASS Mass Analysis for /disk2b/SPECS_SIMS/P-SIMS-LinwenHan/27, XMASS Mass Analysis Constraints

Eor mass $[1]=689.2782480$
zor mass $[2]=711.2626170$

angere $=+1$

Tolevarice $=0.0050000$

DEE min $=-2$

DEE $\max =200$

Max Candidates $=100$

$\begin{array}{lllll}\text { Ator } & \text { \# (min, max) } & \text { Wto (min, max) } \\ \text { C } & 30 & 40 & 0.00 & 100.00 \\ 3 & 25 & 70 & 0.00 & 100.00 \\ 0 & 10 & 20 & 0.00 & 100.00 \\ \operatorname{Na} & 0 & 1 & 0.00 & 100.00\end{array}$

$\begin{array}{lcccccl}\hbar & \text { C } & H & \text { O } & \text { Na } & \text { mass } & \text { error } \\ \star * * & \text { Mass } & \text { Analysis } & \text { for } & \text { mass } 689.2782480 & \\ - & 33 & 46 & 14 & 1 & 689.2779504 & 4.317 e-07 \\ 2 & 35 & 45 & 14 & 0 & 689.2803558 & 3.058 \mathrm{e}-06\end{array}$




\section{$7 \quad$ Xylocarpin F (7)}

IR spectrum

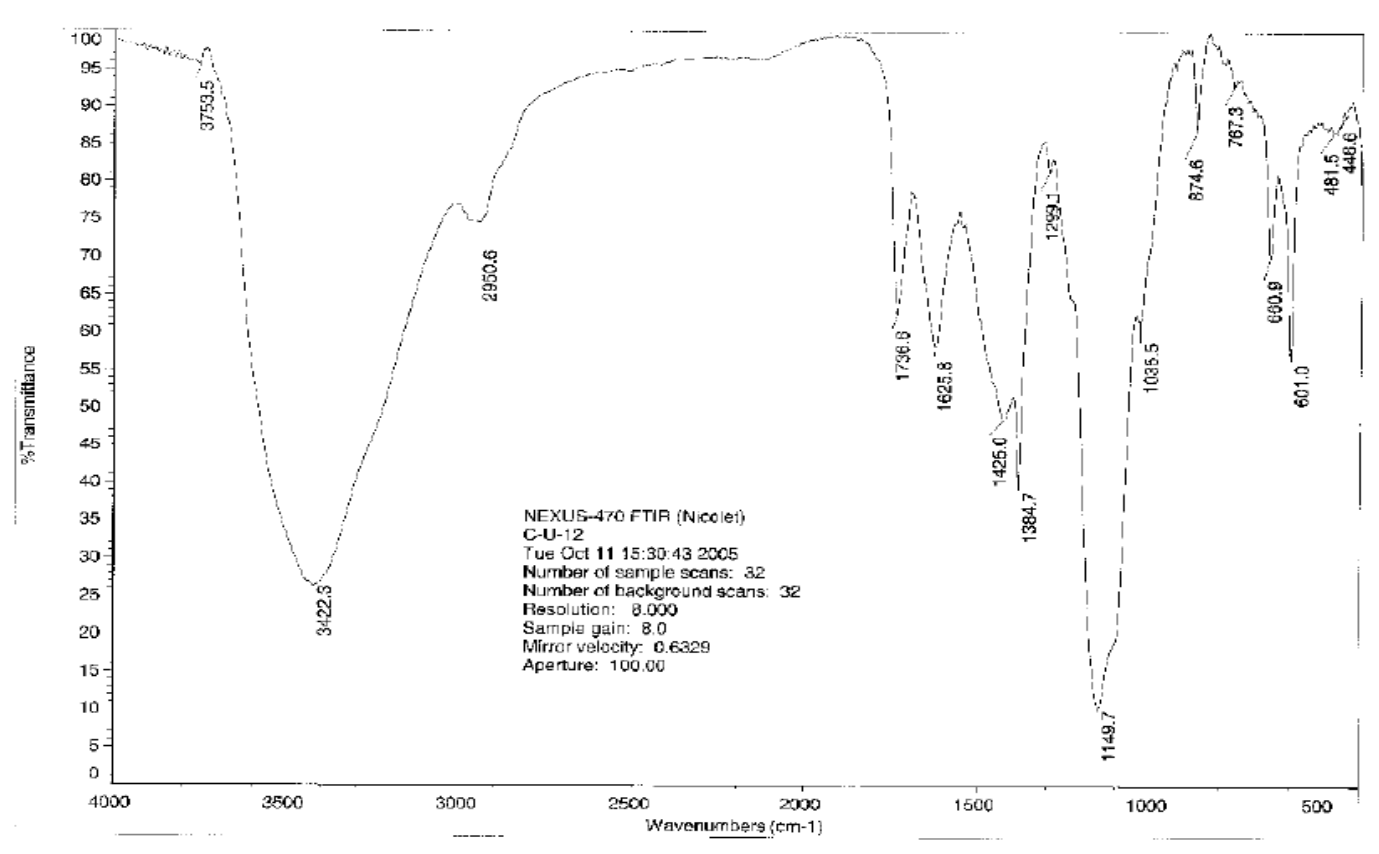

\section{${ }^{1}$ H NMR spectrum}

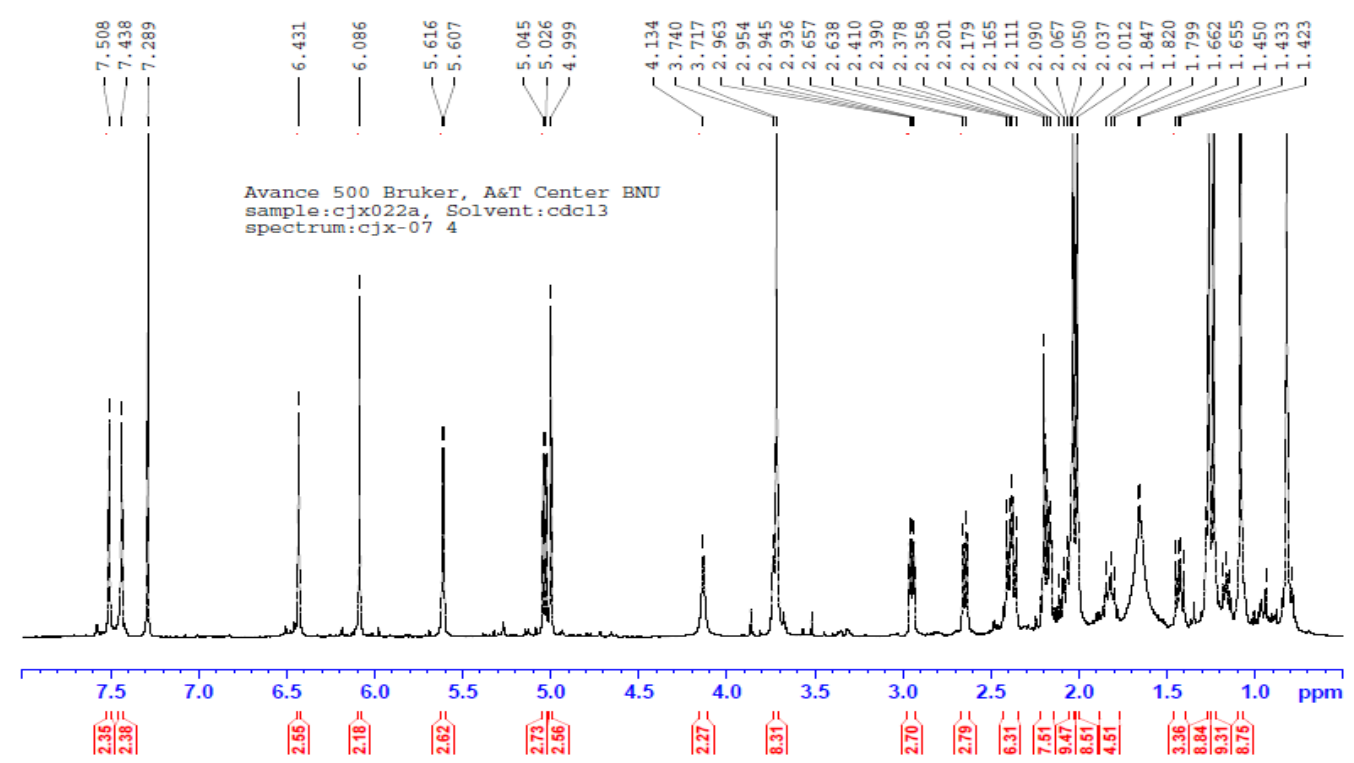




\section{${ }^{13} \mathrm{C}$ and DEPT NMR spectra}

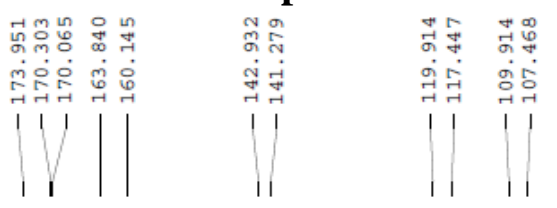

Avance 500 Bruker, A\&T Center BNU sample:cjx022a, Solvent: cdc1

spectrum:cjx-07 11 zgdo
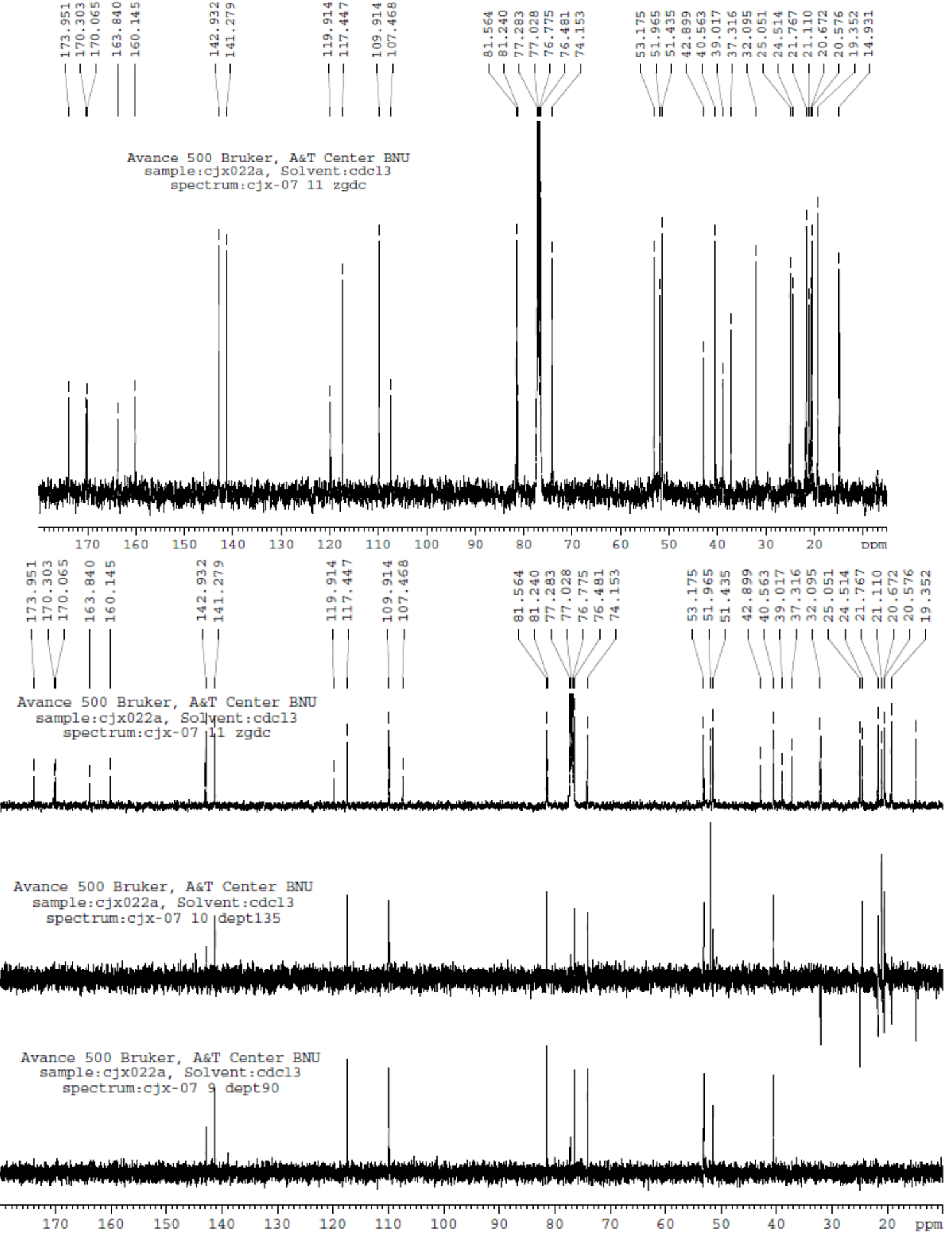


\section{HMQC spectrum}

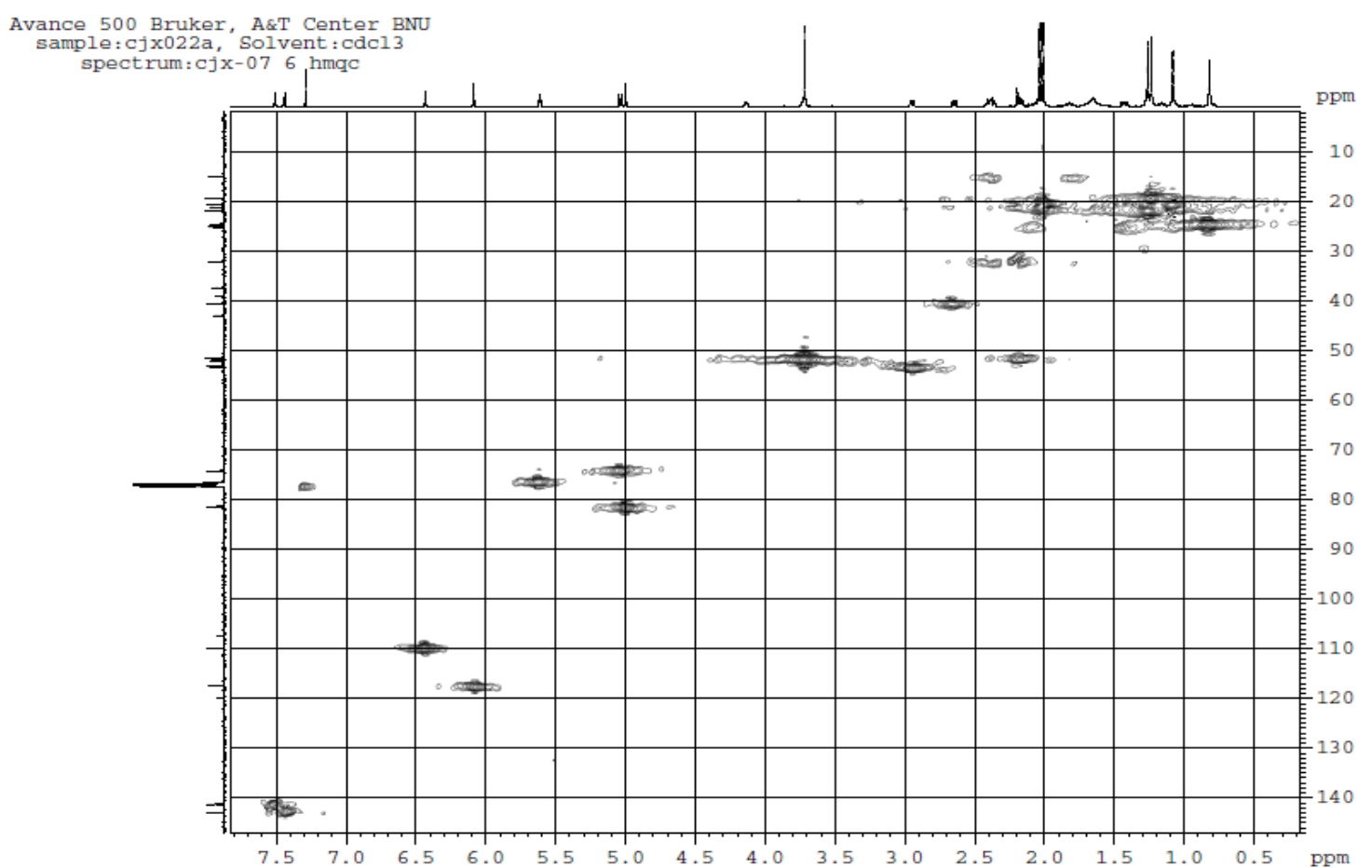

\section{HMBC spectrum}

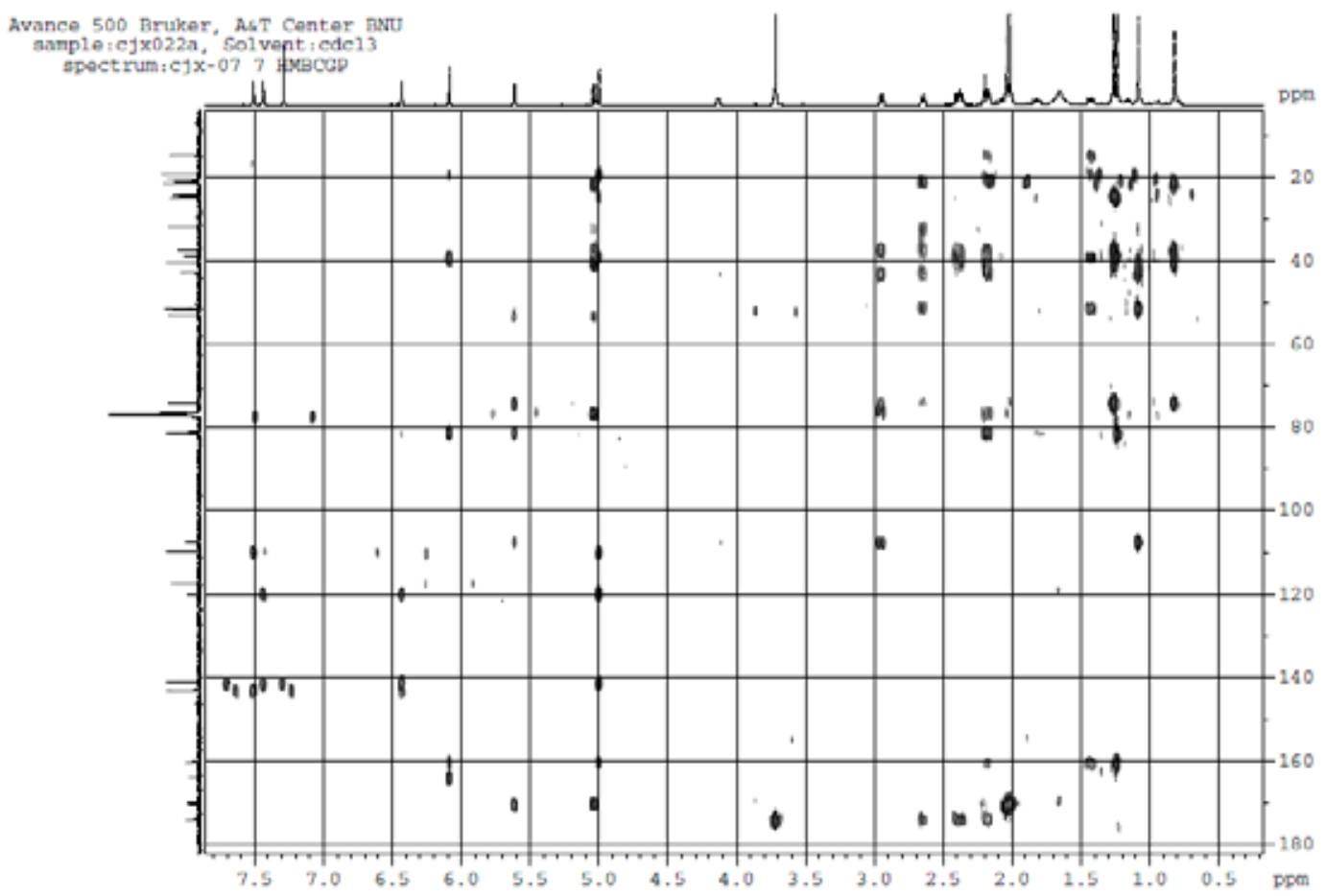




\section{NOESY spectrum}

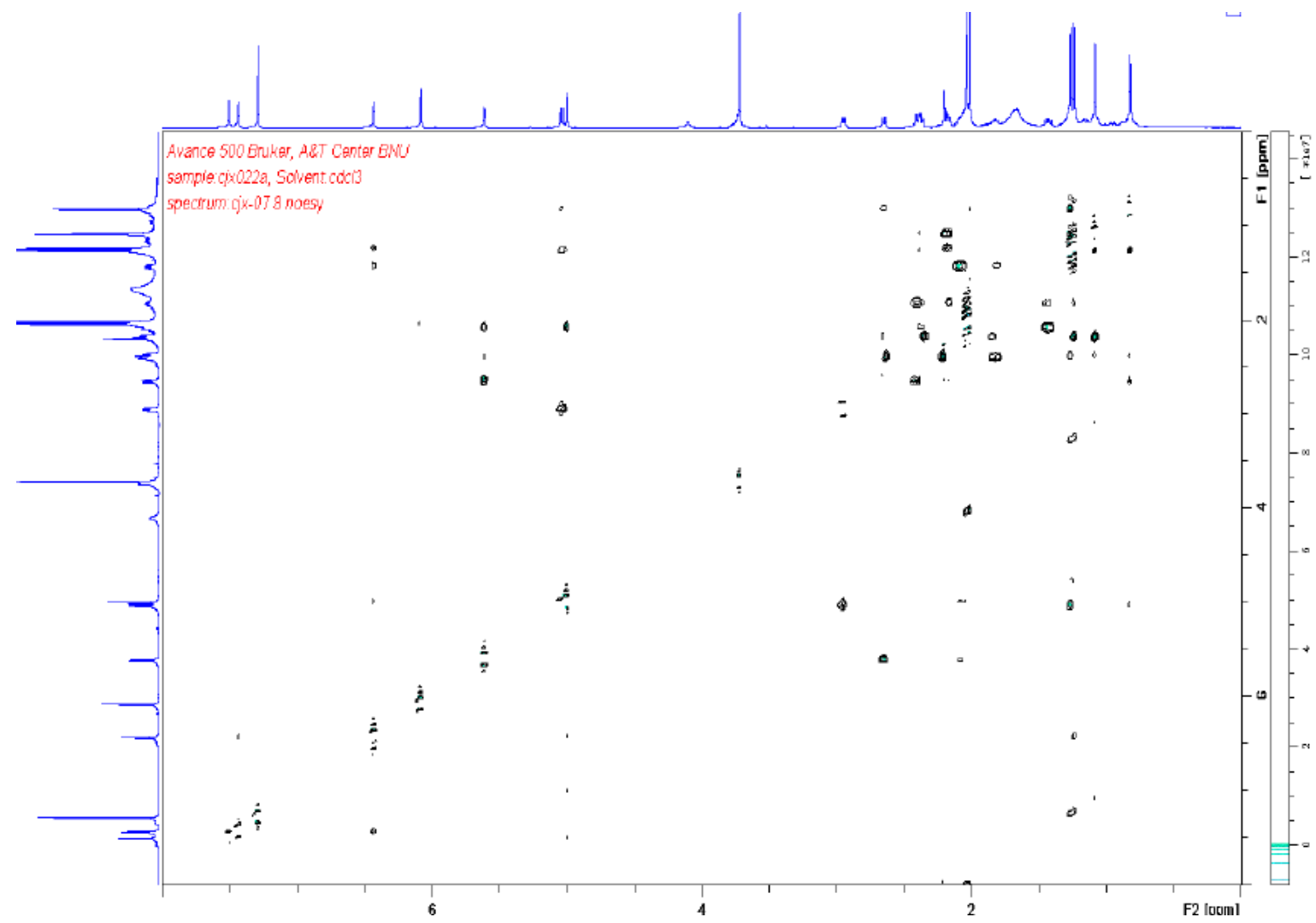

\section{ESIMS spectrum}

ang. 511. 2072005101159349.w1t:

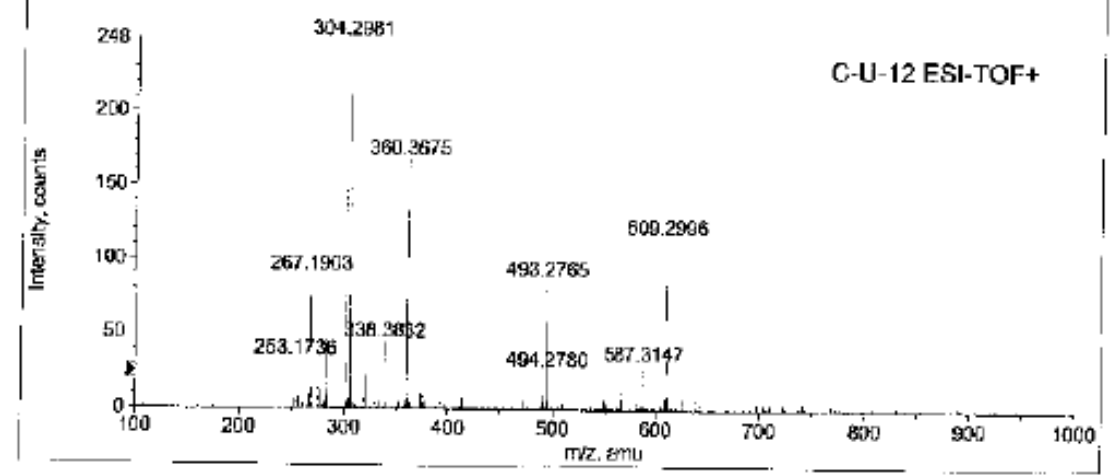




\section{HRSIMS data}

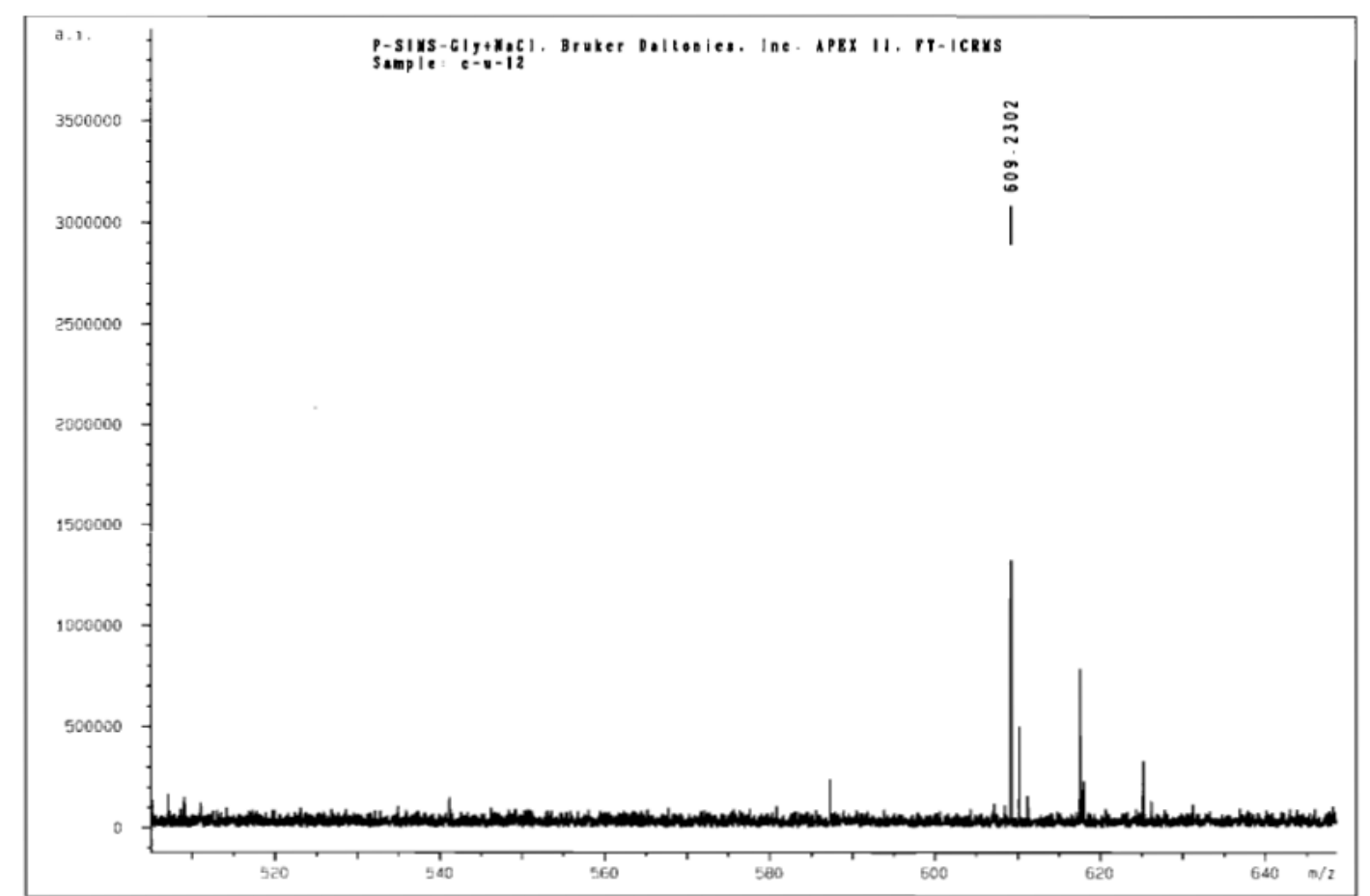

XMASS Mass Analysis for /disk2b/SPECS_SIMS/P-SIMS-LinWenHan/34/pdata/1 XMASS Mass Analysis Constraints

Ion $\mathrm{mass}=609.2301500$

Charge $=+1$

Tolerance $=0.0030000$

$\mathrm{DBE} \min =-2$

$\mathrm{DBE} \max =200$

Max Candidates $=100$

Azom \#(min, max)

C 20 40

H $20 \quad 55$

$\begin{array}{lll}10 & 20\end{array}$

we \& $(\min , \max )$

$0.00 \quad 100.00$

$0.00 \quad 100.00$

$\mathrm{Na}$

1

$0.00 \quad 100.00$

$0.00 \quad 100.00$

\# $\begin{array}{lcccc}\mathrm{C} & \mathrm{H} & \mathrm{O} & \mathrm{Na} & \mathrm{mass} \\ \text { Mass } & \text { Analysis for mass } & 609.2301500\end{array}$

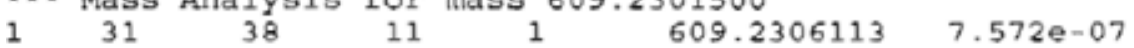




\section{Xylocarpin G (8)}

IR spectrum

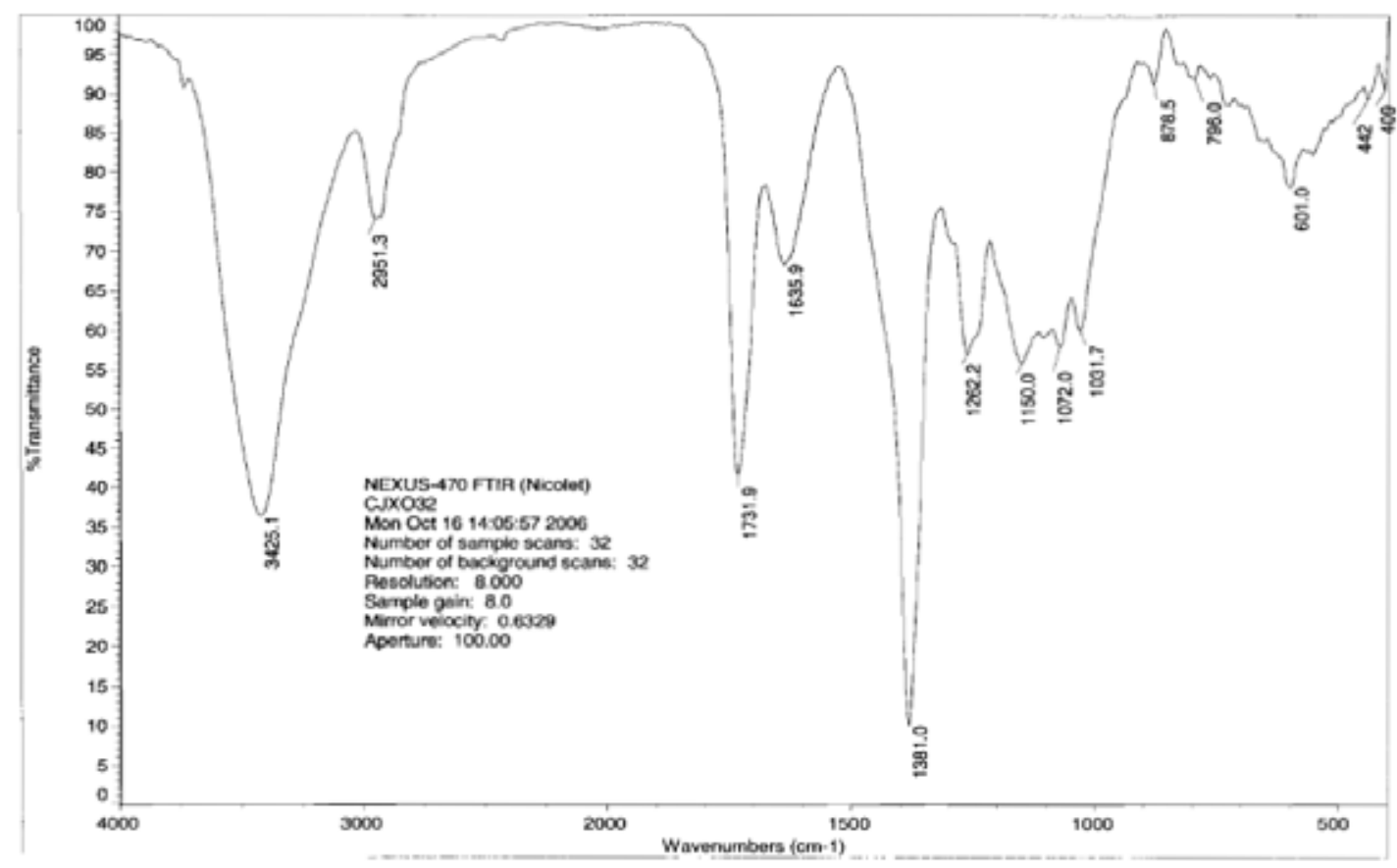

\section{${ }^{1}$ H NMR spectrum}

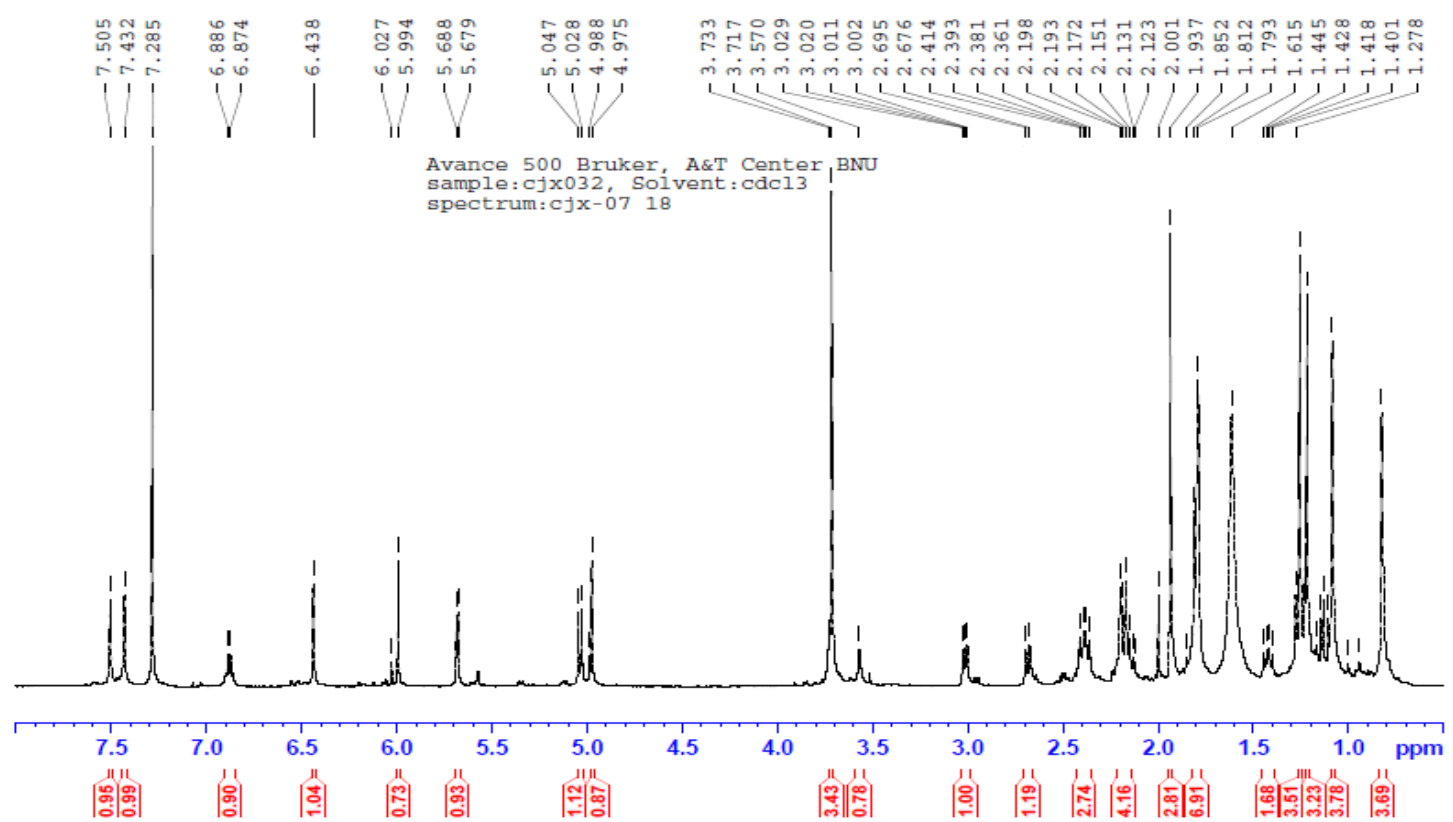




\section{${ }^{13} \mathrm{C}$ and DEPT NMR spectra}
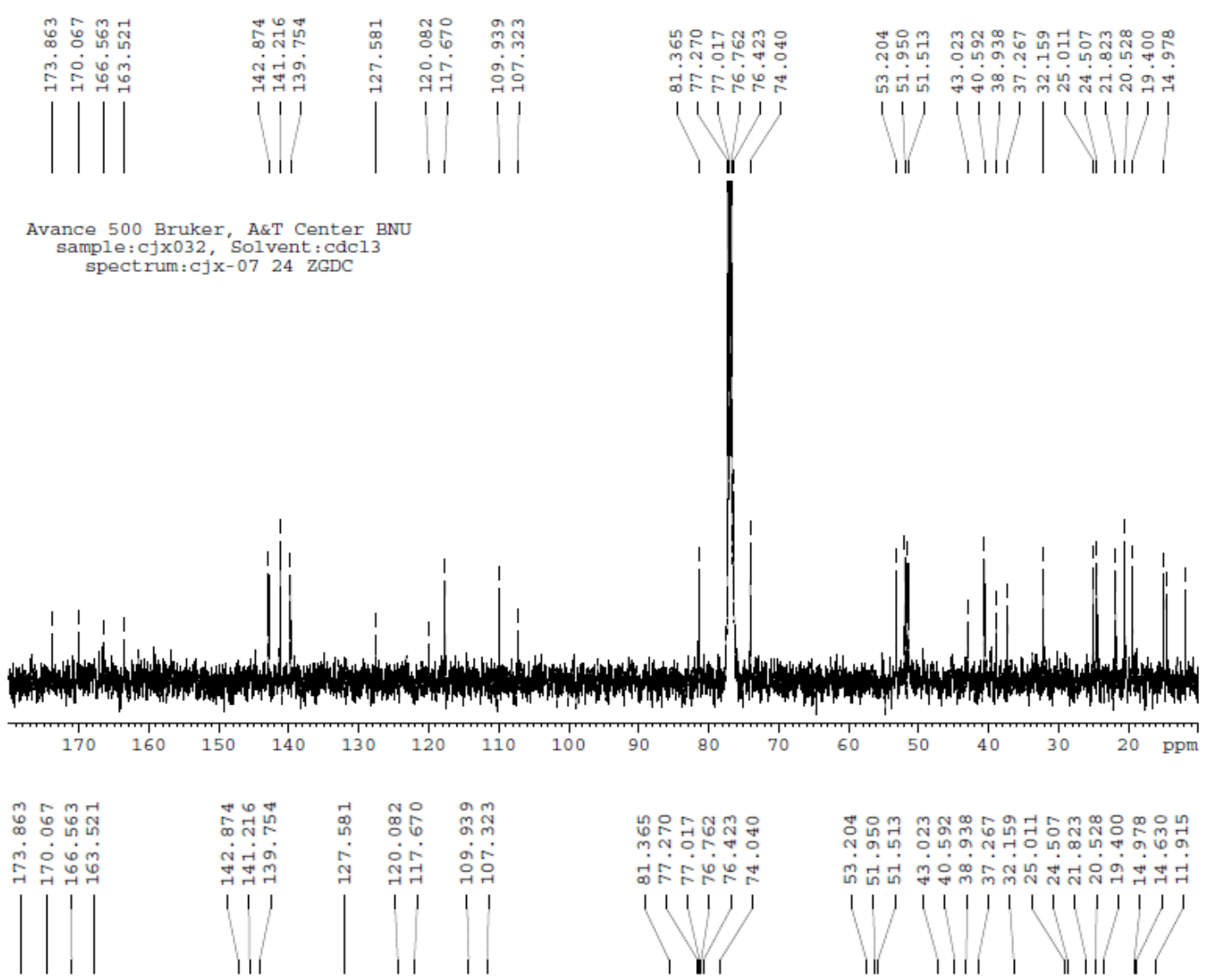

Avance 500 Bruker, A\&T Center BNU sample:cjx032, Solvent: cdcl3 spectrum: cjix-07 24 ZGDC
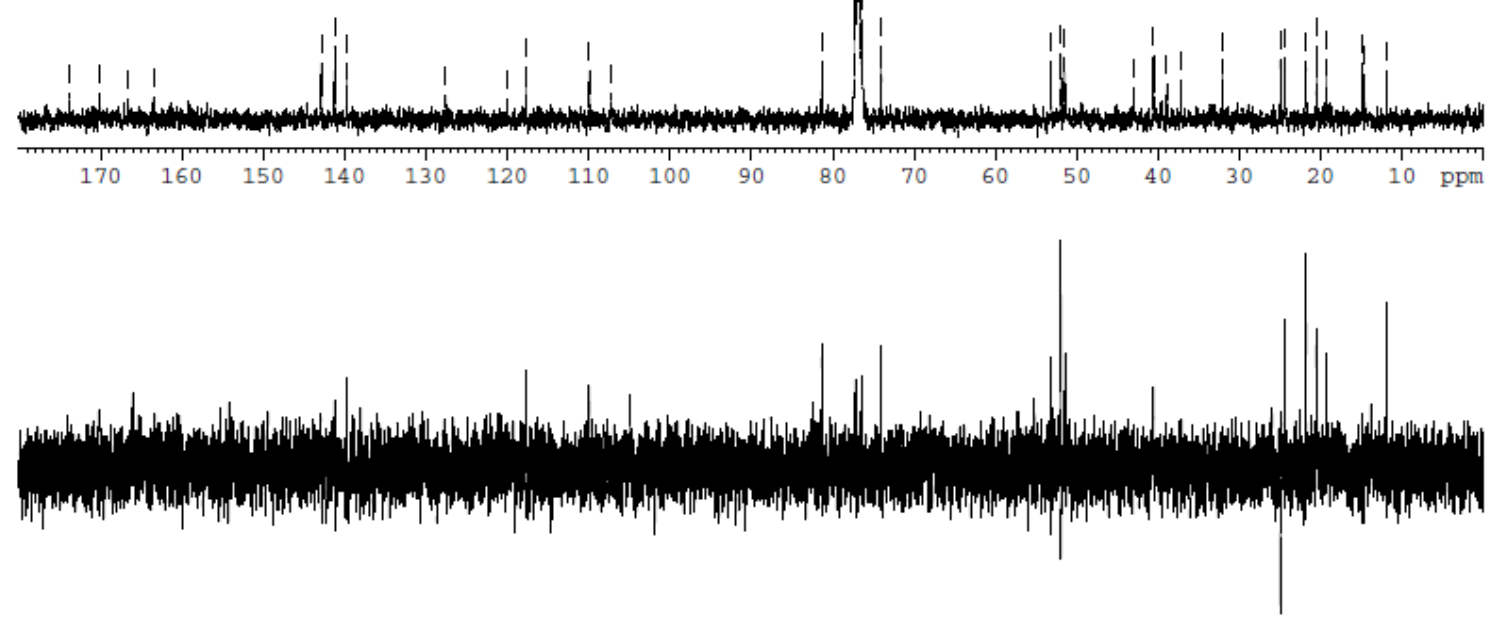

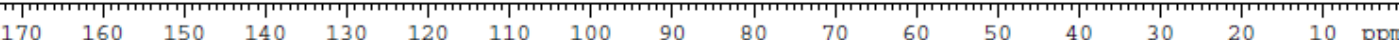




\section{HMQC spectrum}

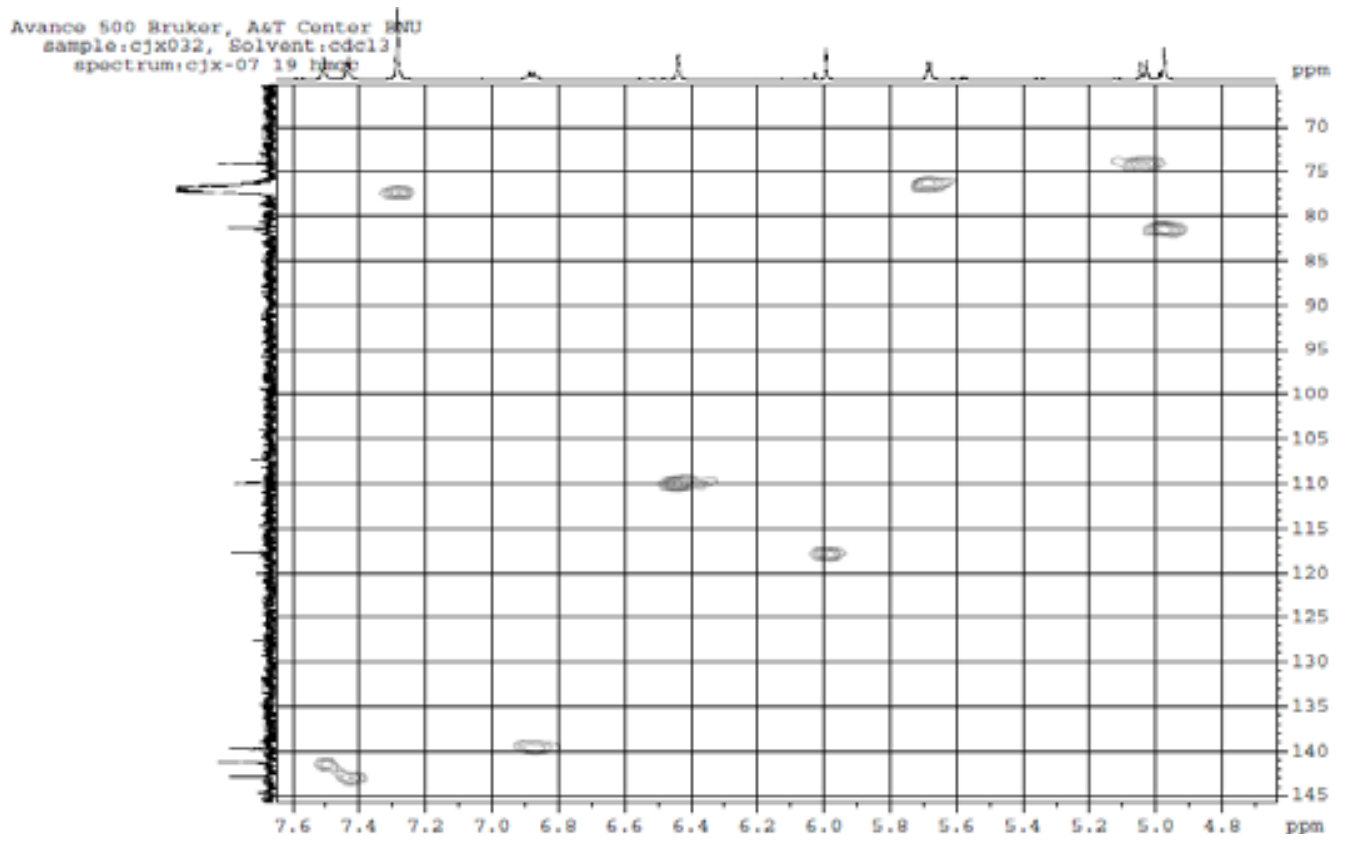

\section{HMBC spectrum}

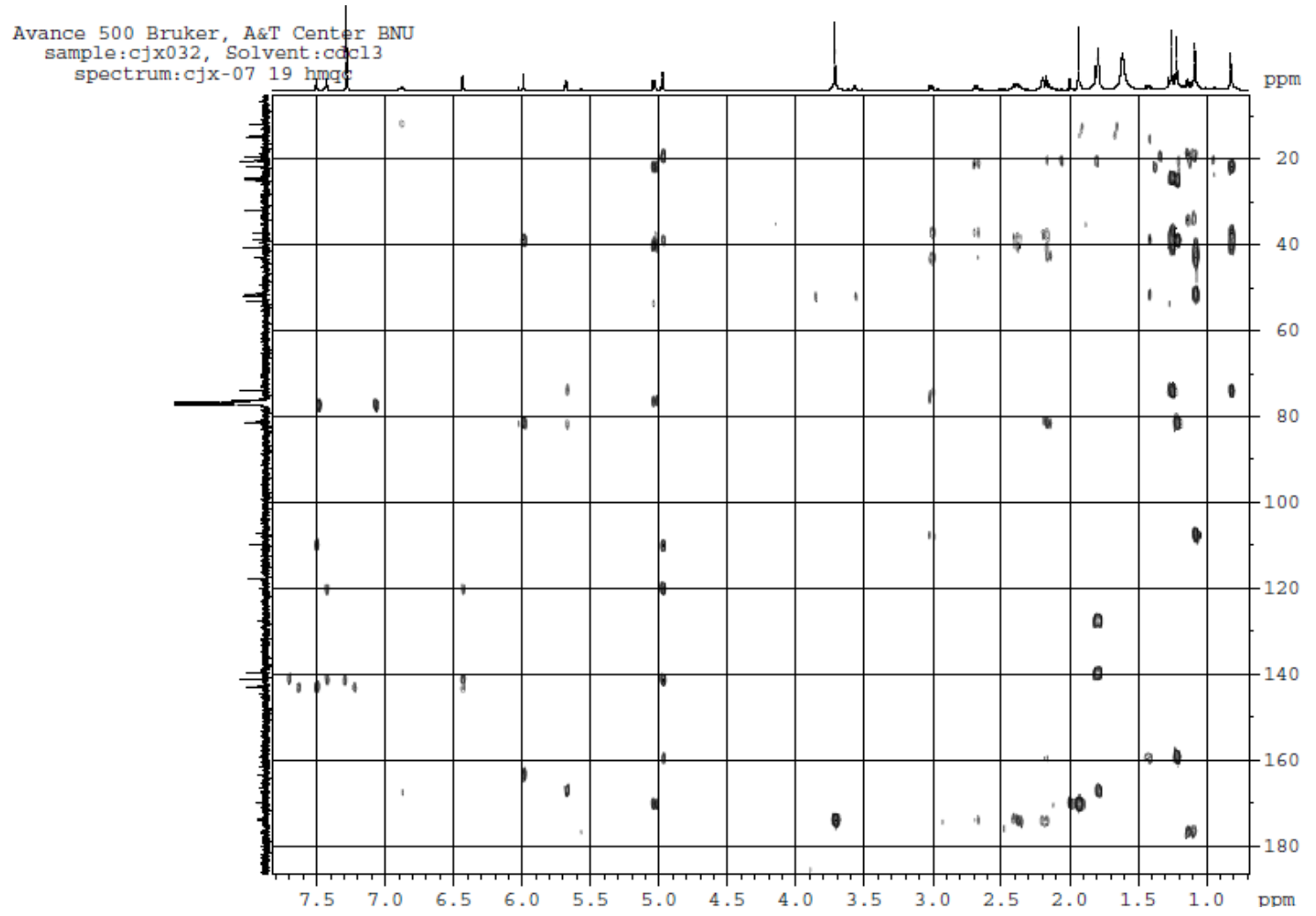




\section{HRSIMS spectrum}

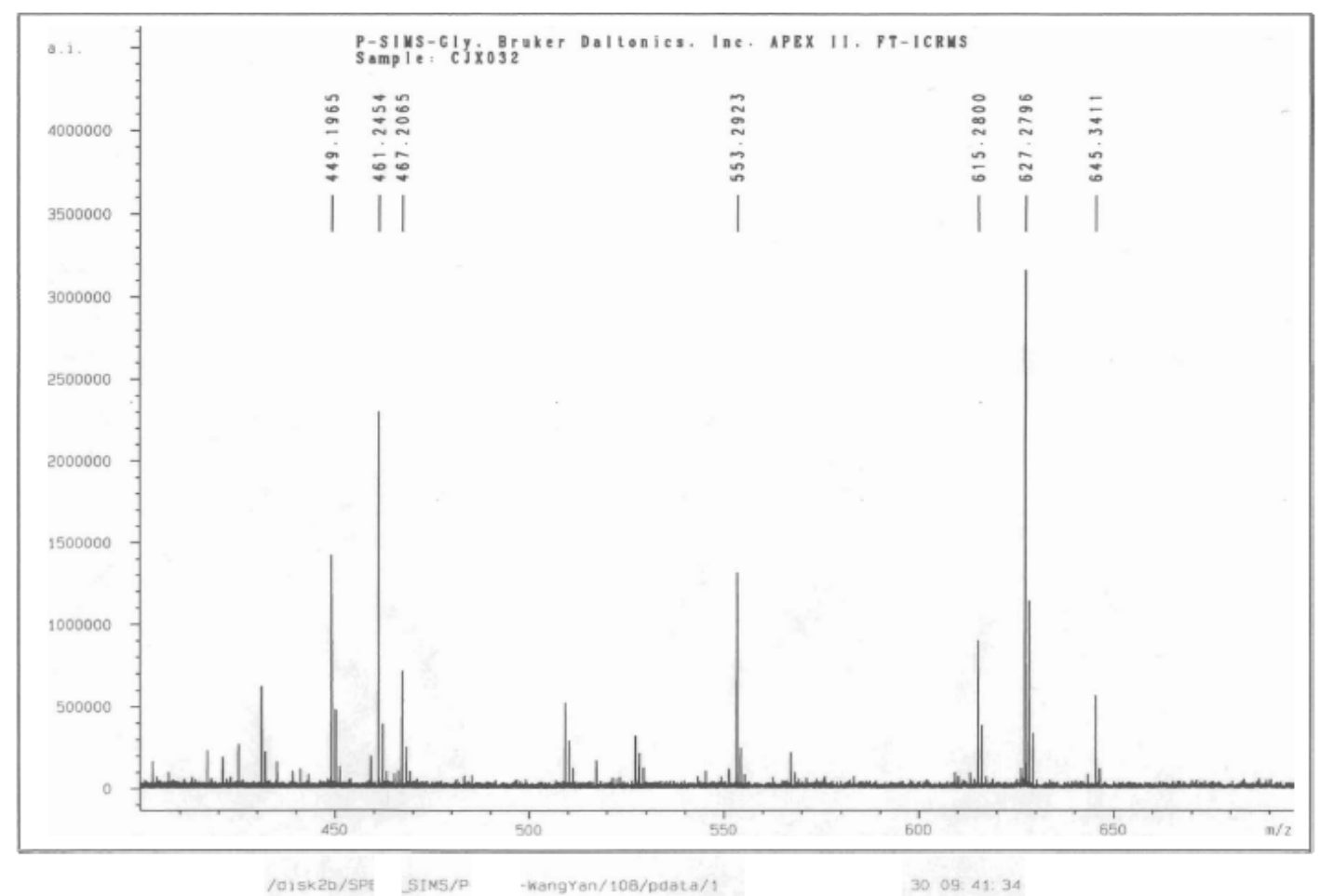

XMASS Mass Analysis for /disk2b/SPECS_SIMS/P-SIMS-WangYan/108/pdaca/1/massanal XMASS Mass Analysis Constraint.s

Ion mass $=627.2796360$

Charge - +1

Tolerance $=0.0030000$

$\mathrm{DBE} \min =-2$

$D B E \max =200$

Max Candidates $=100$

Atom \#(min, max) Wt (min, $\max )$

$\begin{array}{lllll}\text { C } & 30 & 36 & 0.00 & 100.00\end{array}$

$\begin{array}{llll}30 & 50 & 0.00 & 100.00\end{array}$

$\begin{array}{lllll}0 & 5 & 15 & 0.00 & 100.00\end{array}$

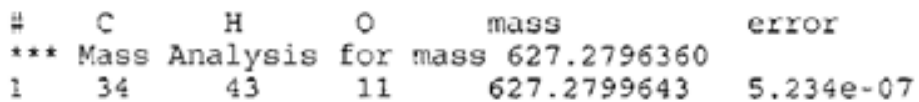




\section{Xylocarpin H (9)}

\section{IR spectrum}

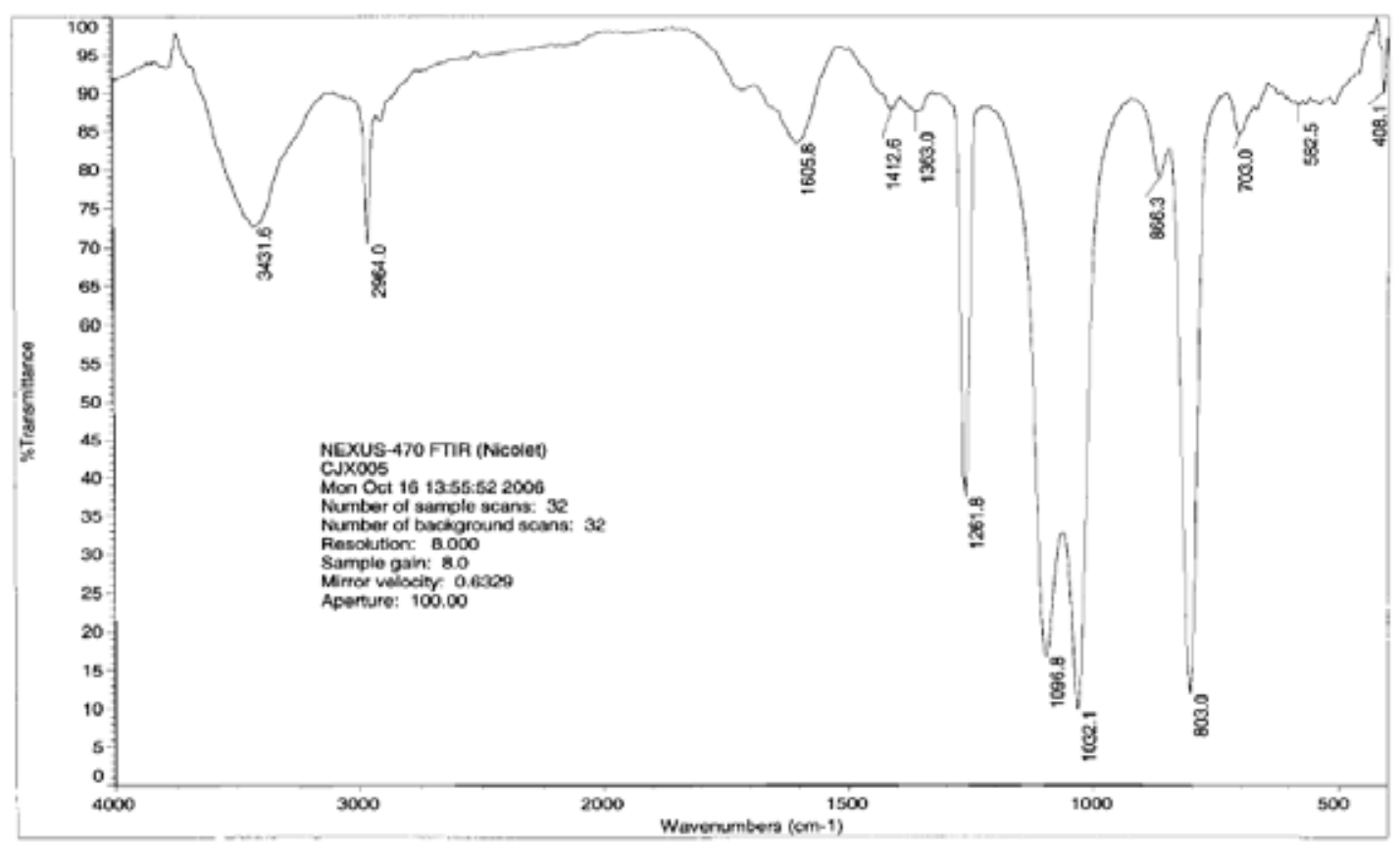

\section{${ }^{1}$ H NMR spectrum}

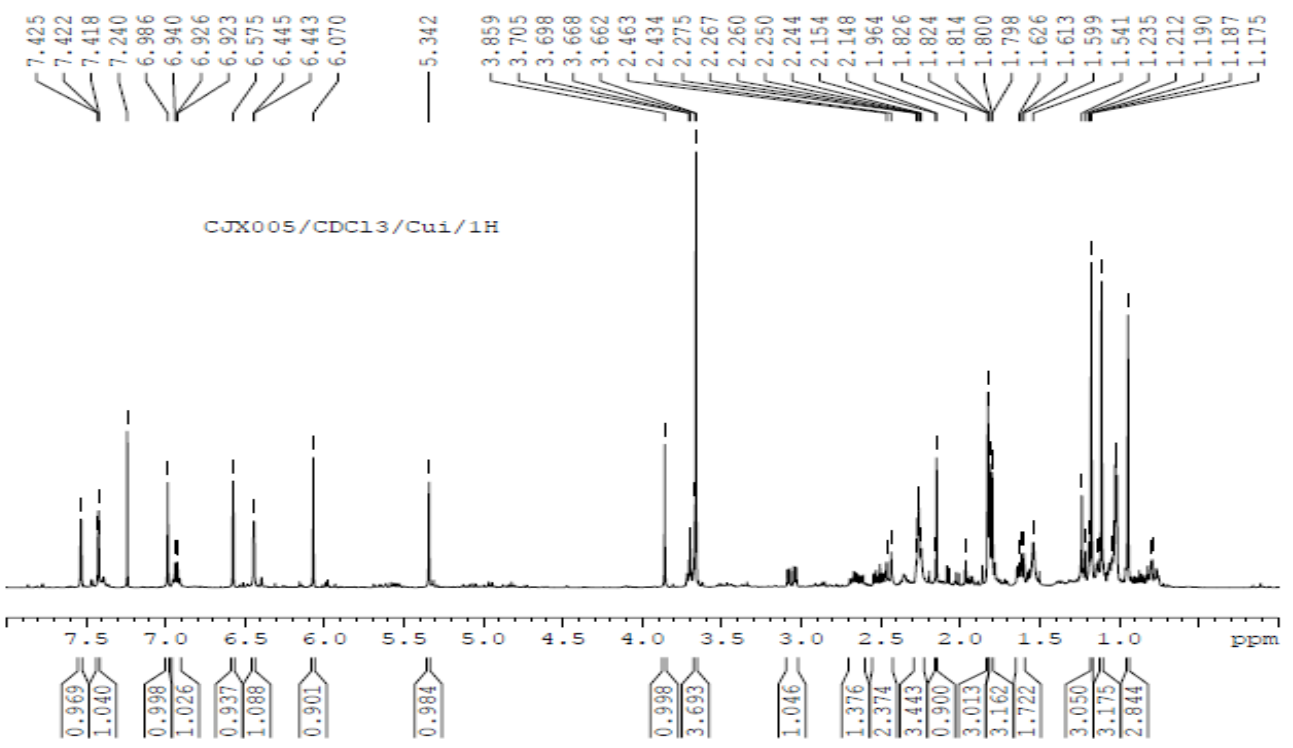




\section{${ }^{13} \mathrm{C}$ and DEPT NMR spectra}
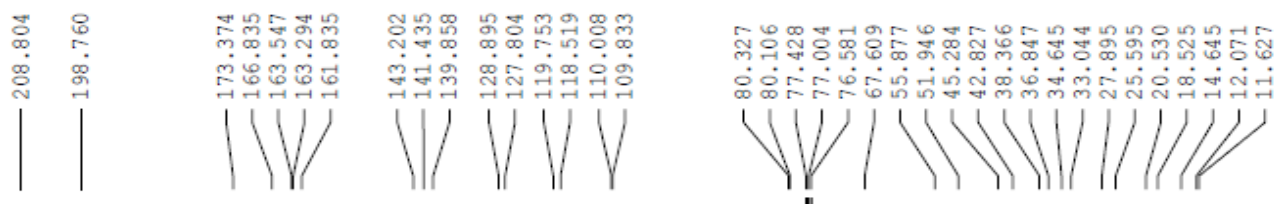

CJX005/CDC13/Cui/13C
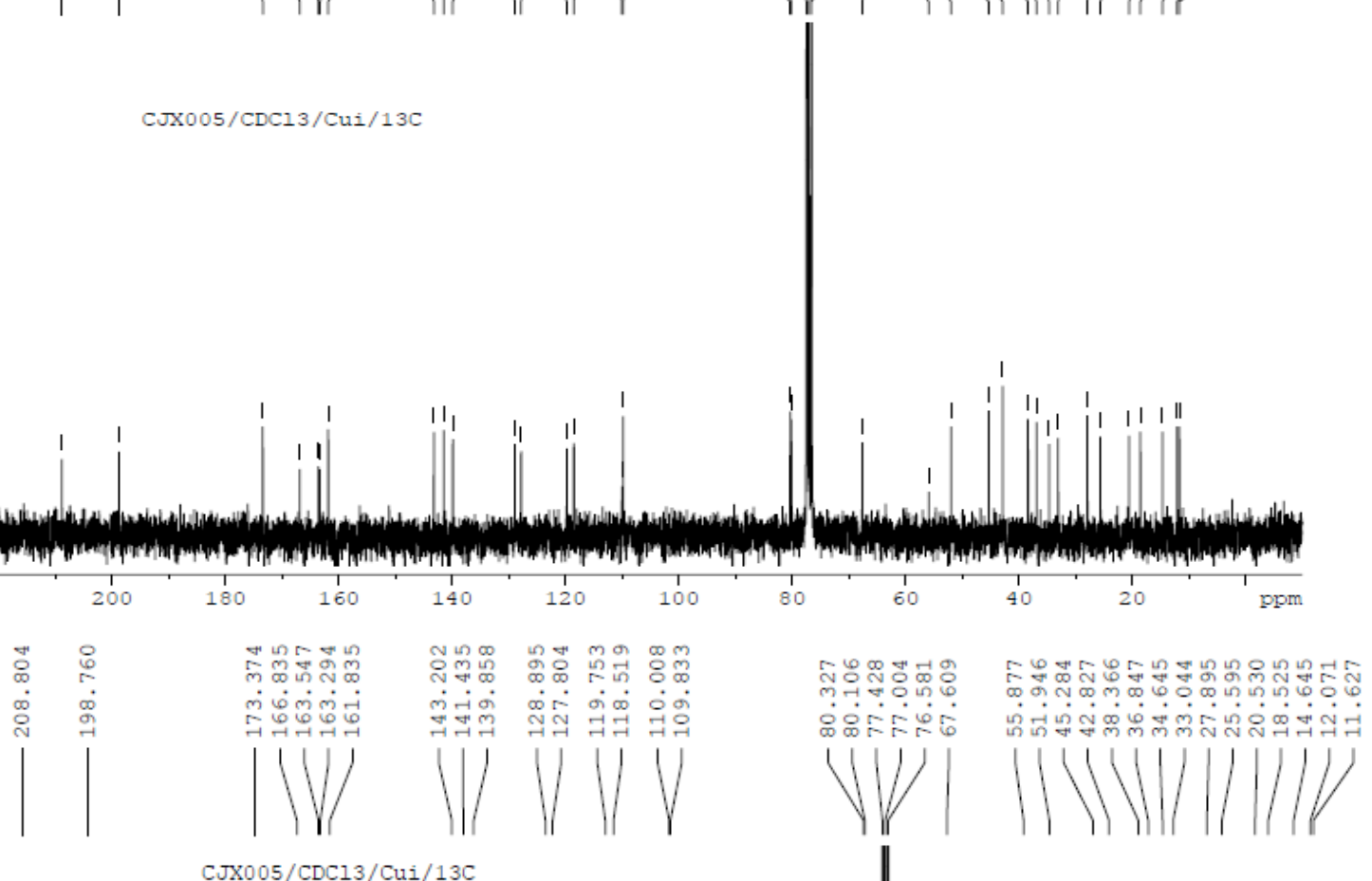

CJX005/CDC13/Cui/13C

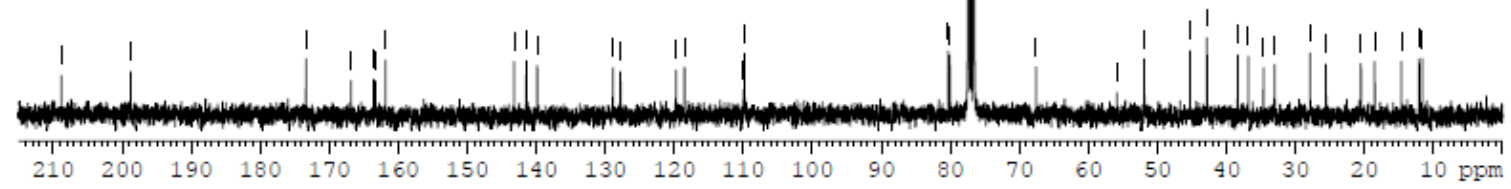

CJX005/CDC13/Cui/DEPT135

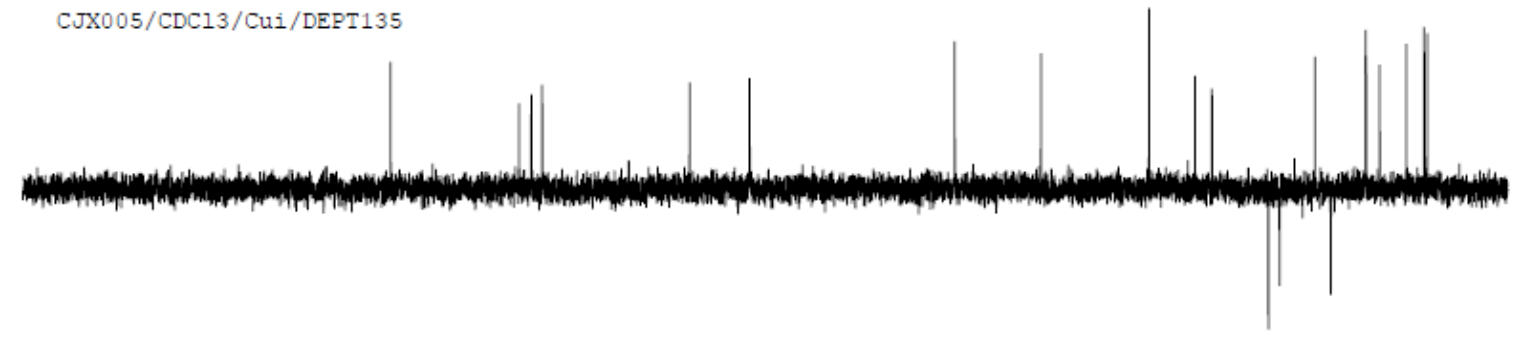

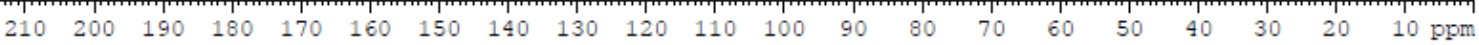




\section{HMQC spectum}

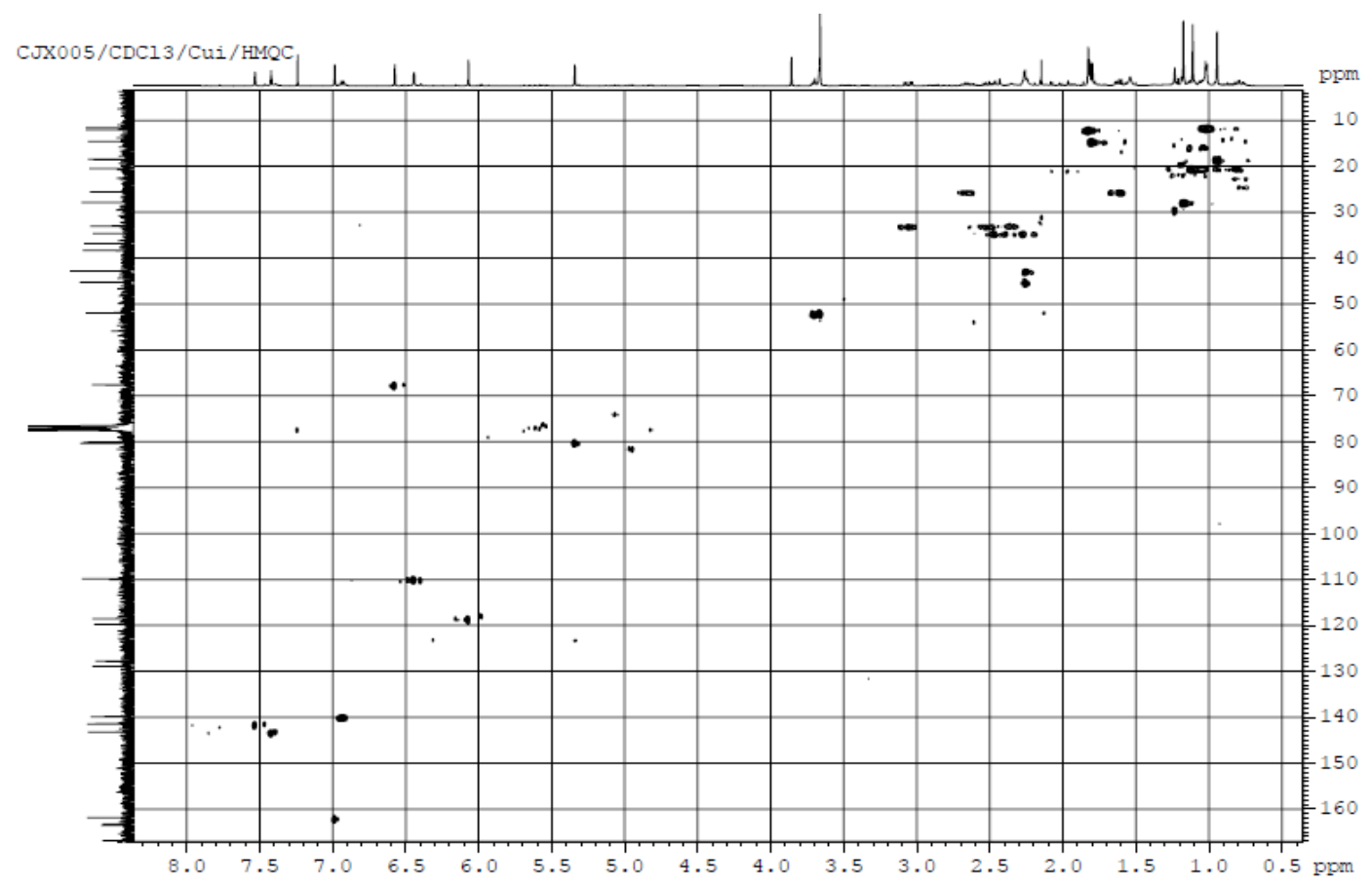

\section{HMBC spectrum}

CJX005/CDC13/Cui/HMBC

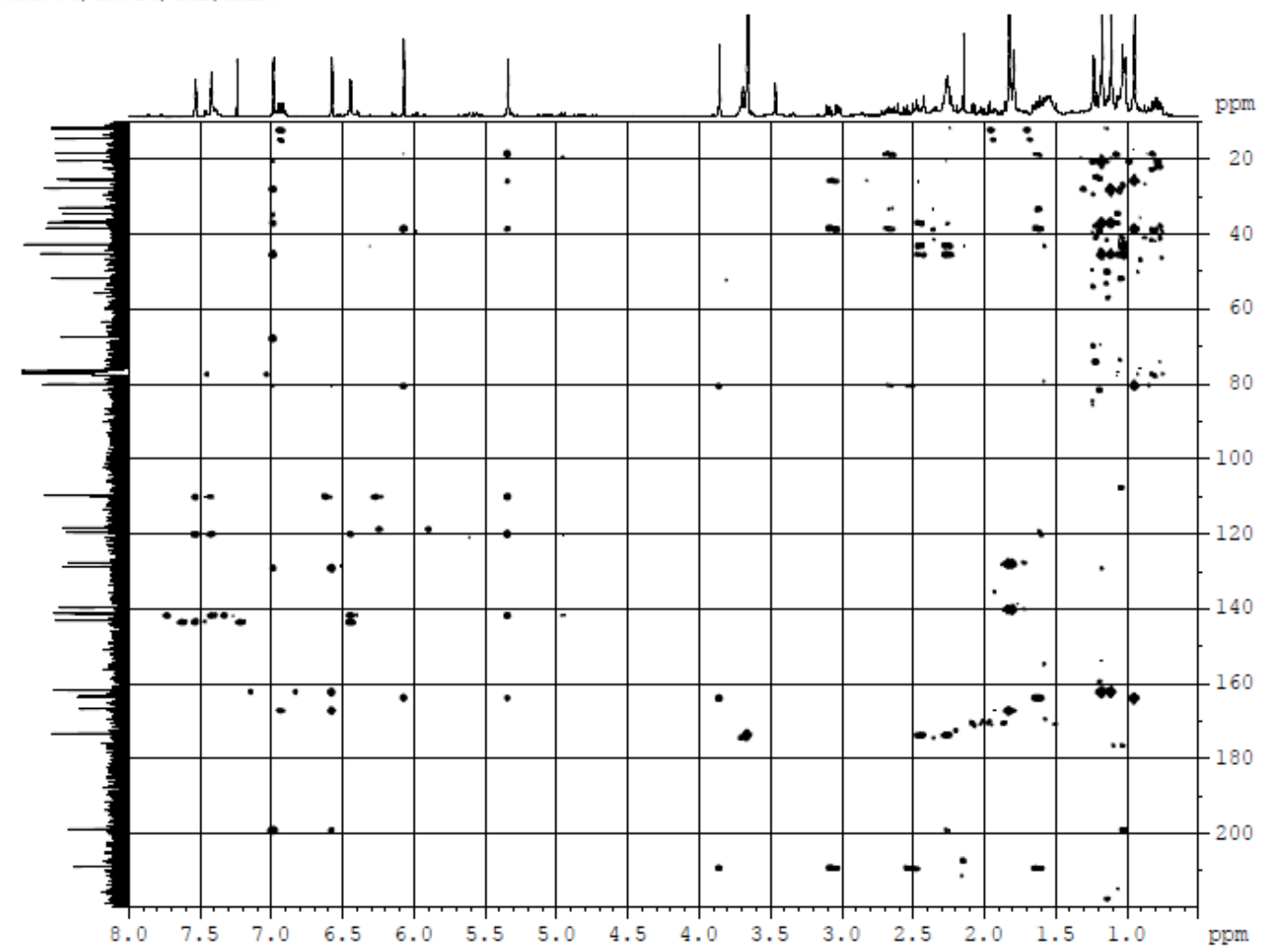




\section{NOESY spectrum}

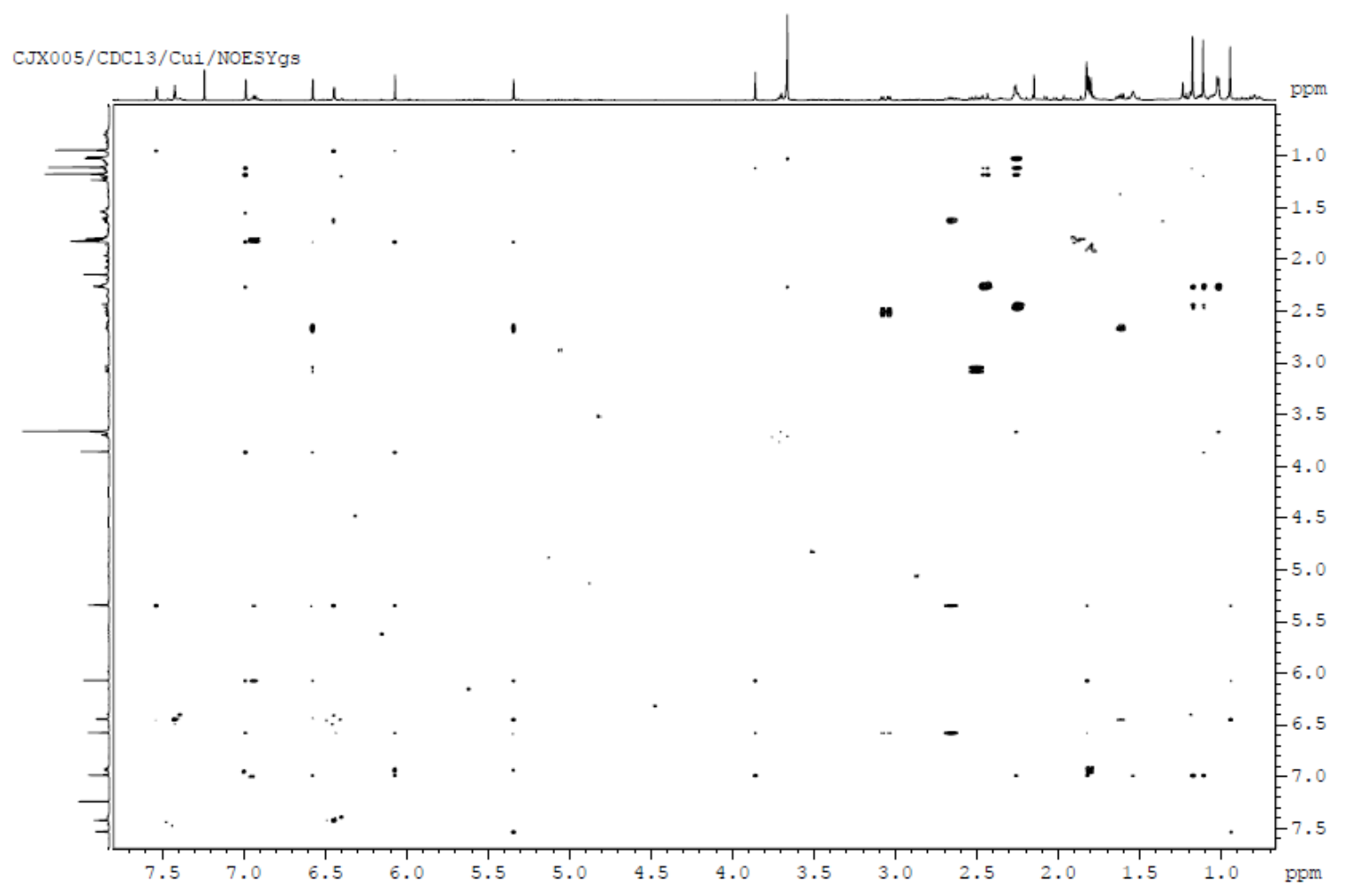

\section{ESIMS spectrum}

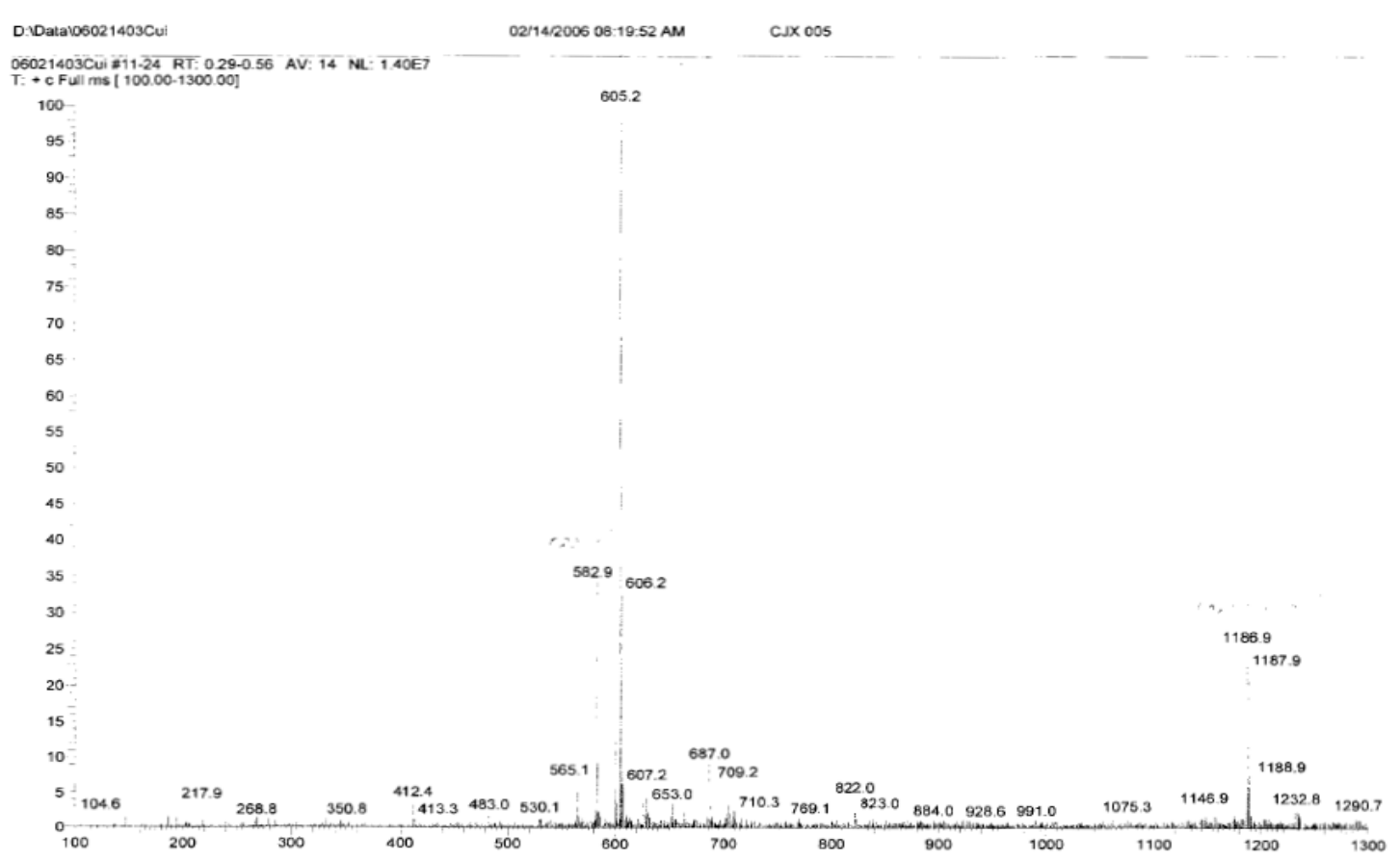




\section{HRSIMS spectrum}

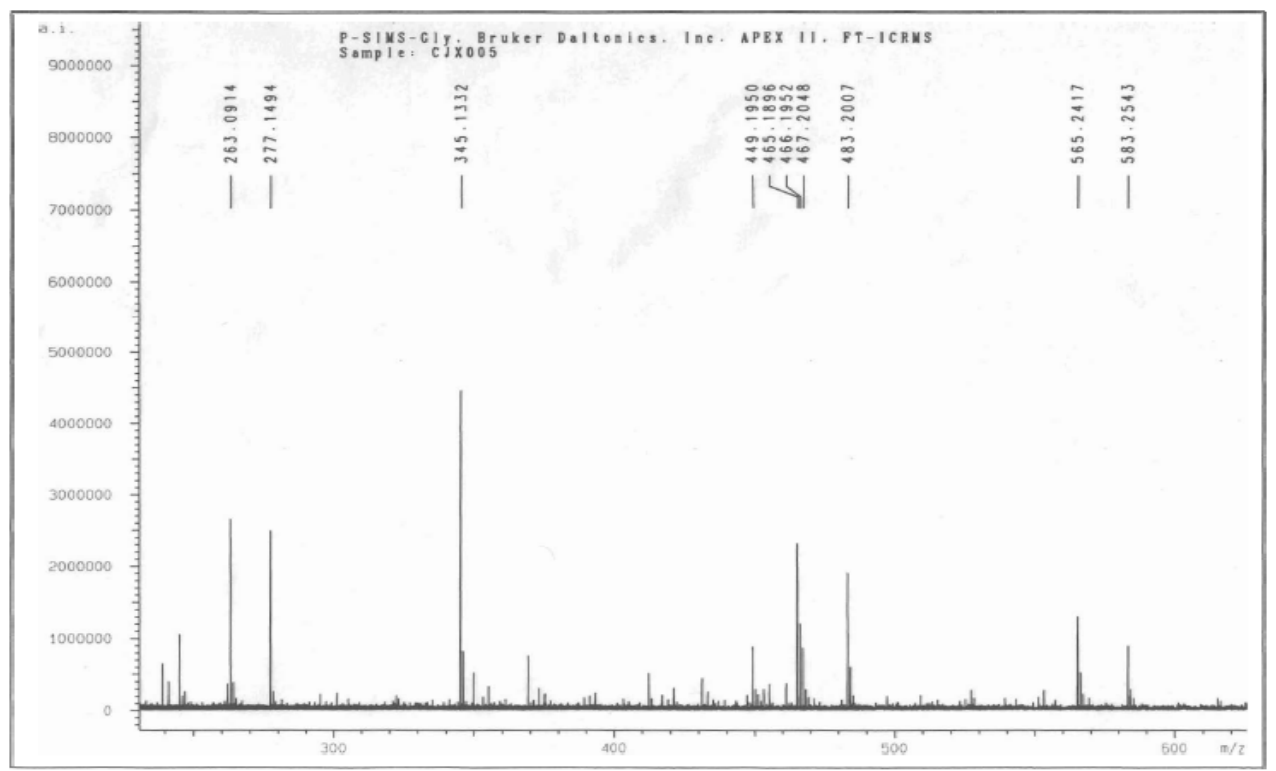

XMASS Mass Analysis for /disk2b/SPECS_SIMS/P-SIMS-WangYan/113/pdata/1/massana: XMASS Mass Analysis Constraints

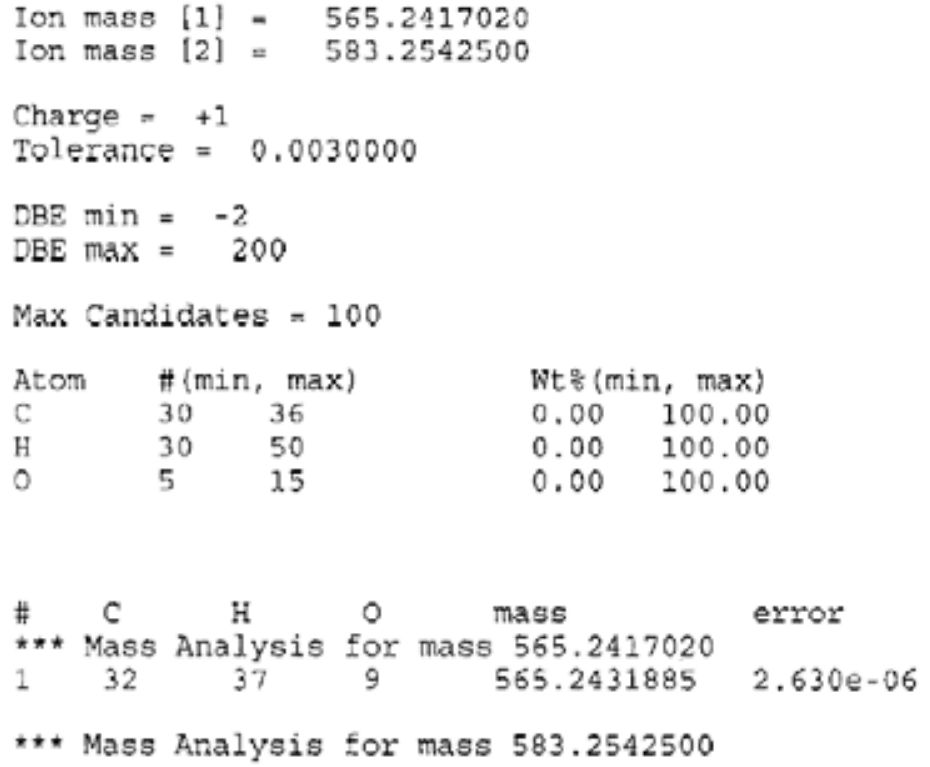




\section{Xylocarpin I (10)}

IR spectrum

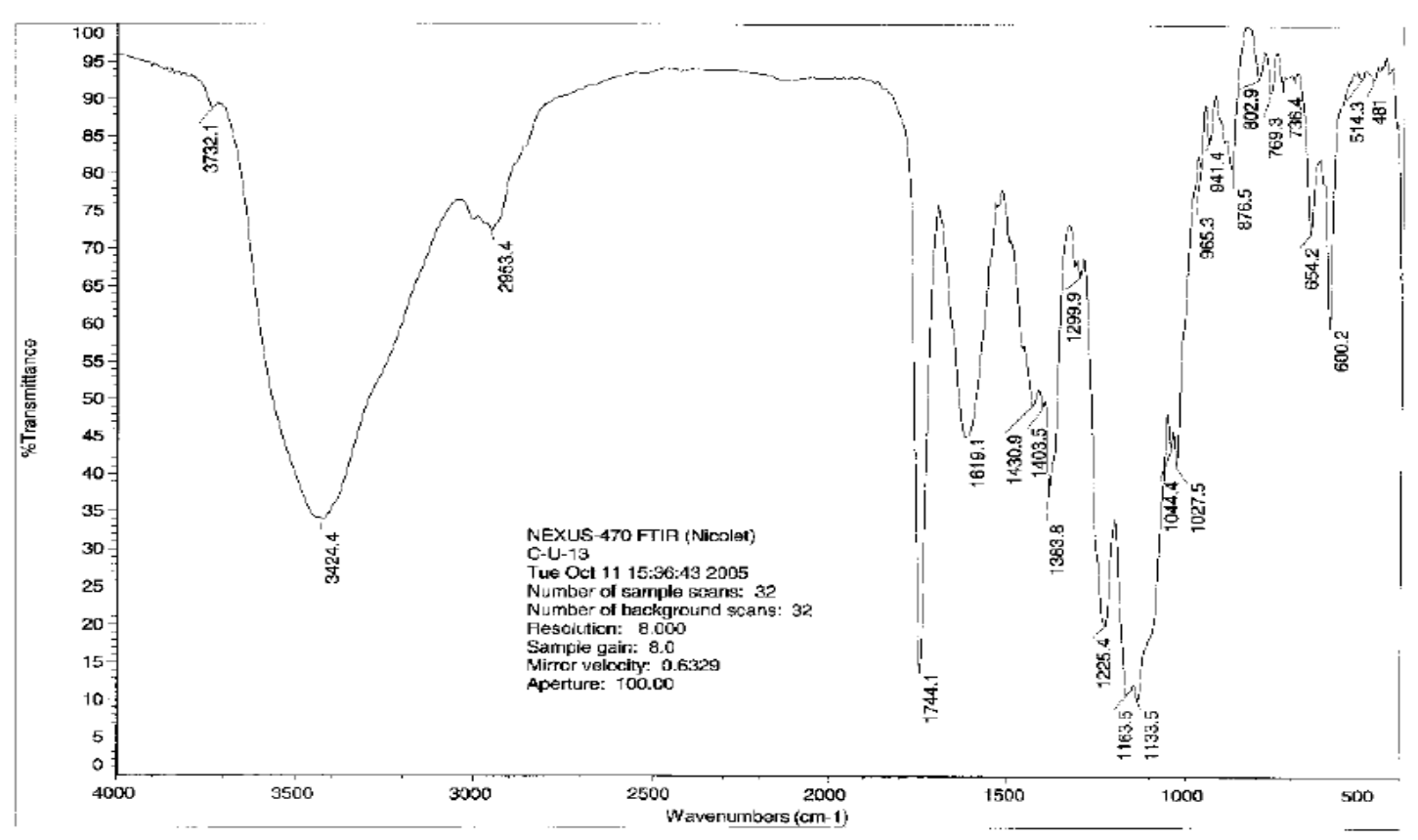

\section{${ }^{1}$ H NMR spectrum}

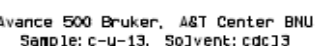

nple: (13. Solvent: cac 3

高

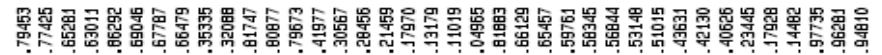

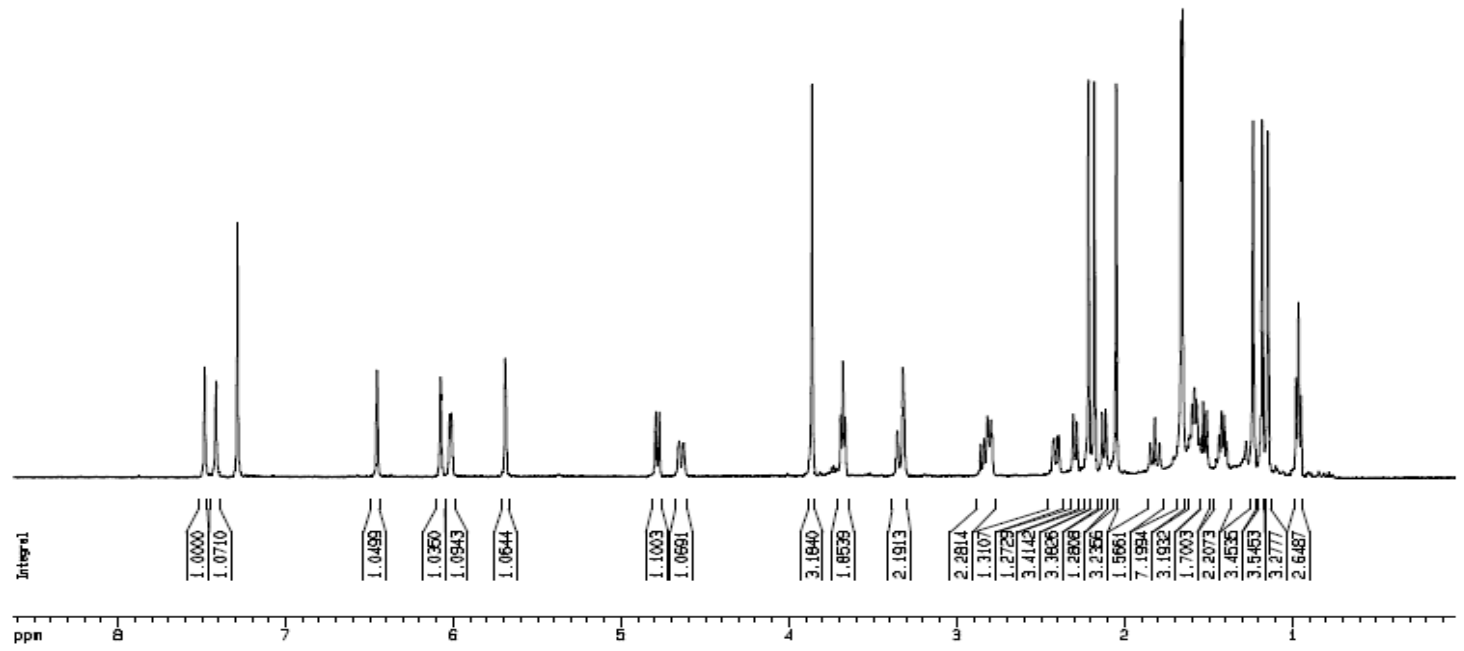




\section{${ }^{13} \mathrm{C}$ and DEPT NMR spectra}

Avance 500 Eruker, $\triangle 8$ T Center BNU

Sanple: c-u-13, So]vent: cac]J

Cul-10 11 13C

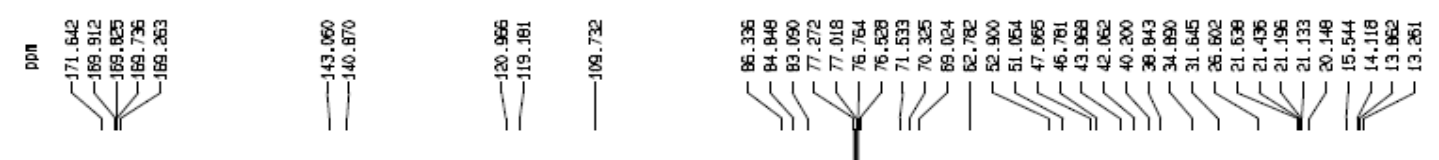
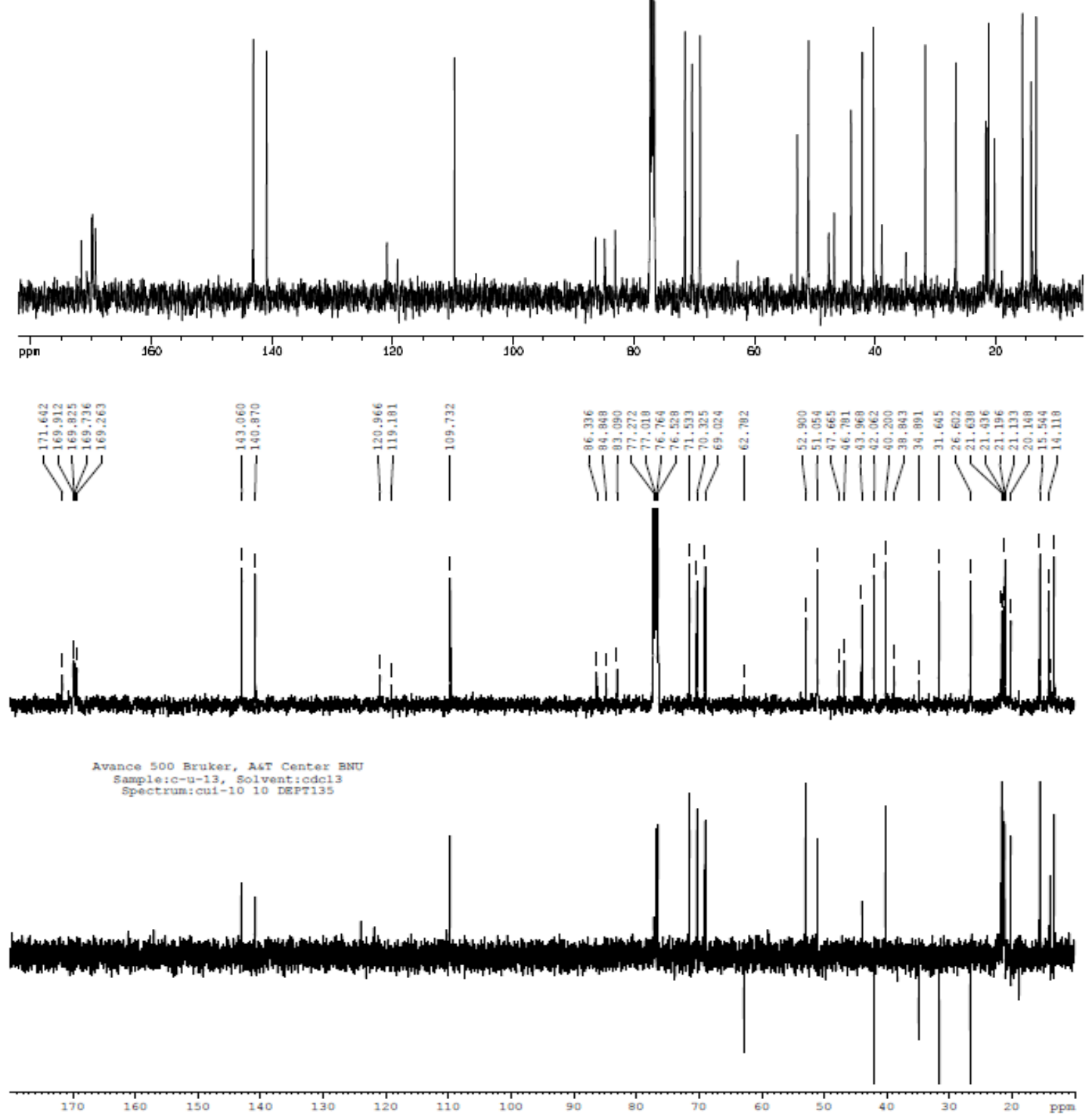


\section{HMQC spectrum}

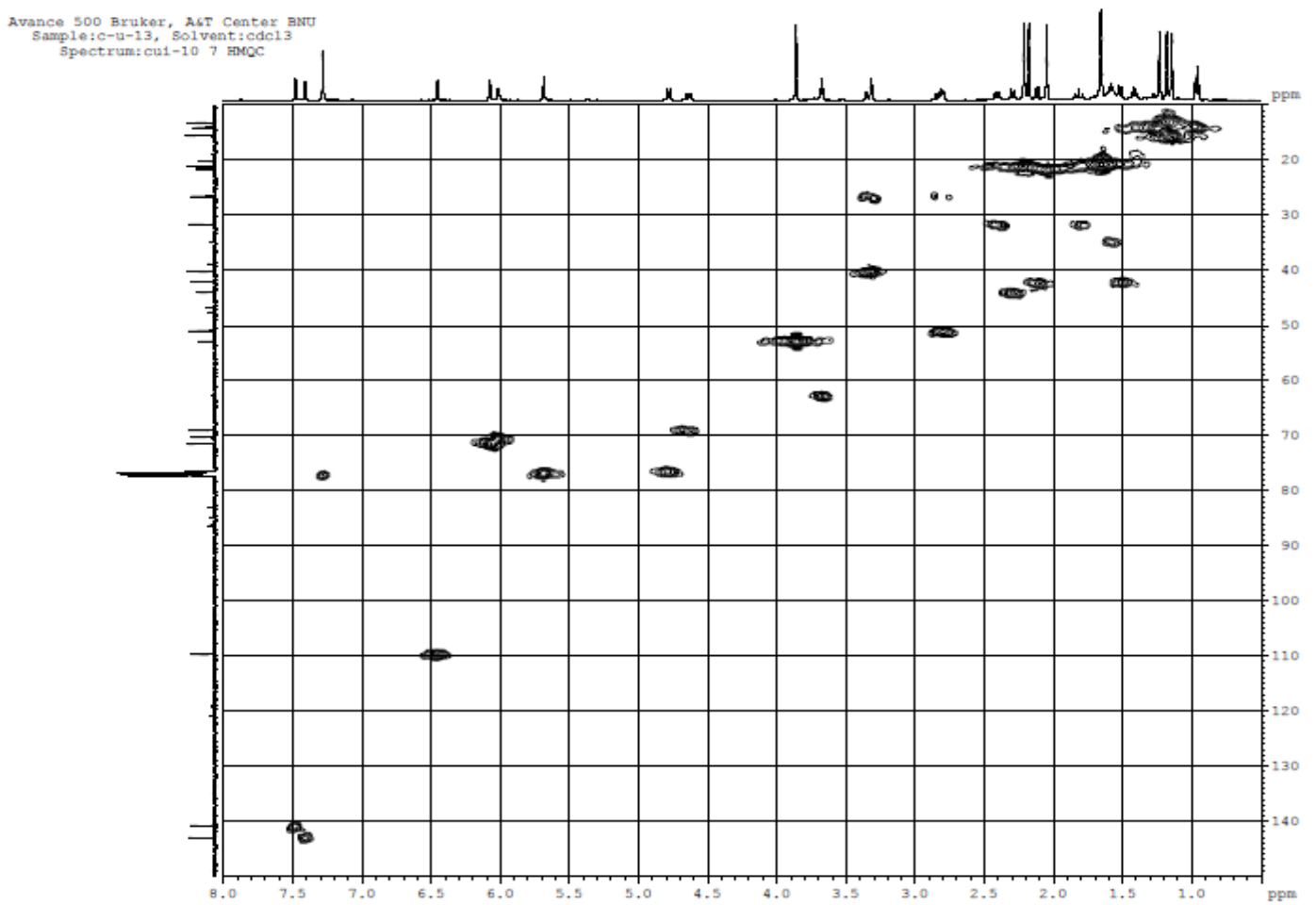

\section{HMBC spectrum}

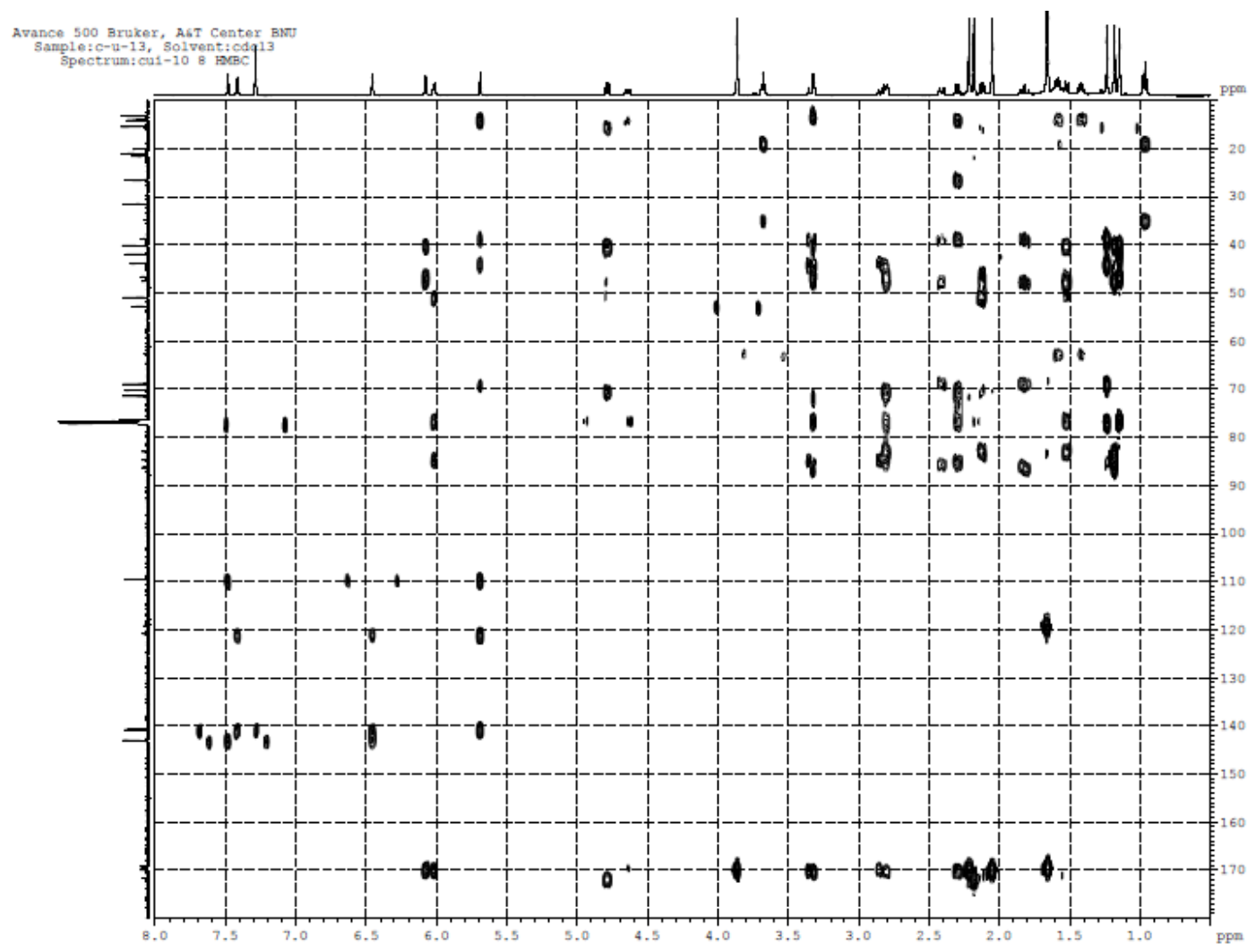




\section{NOESY spectrum}

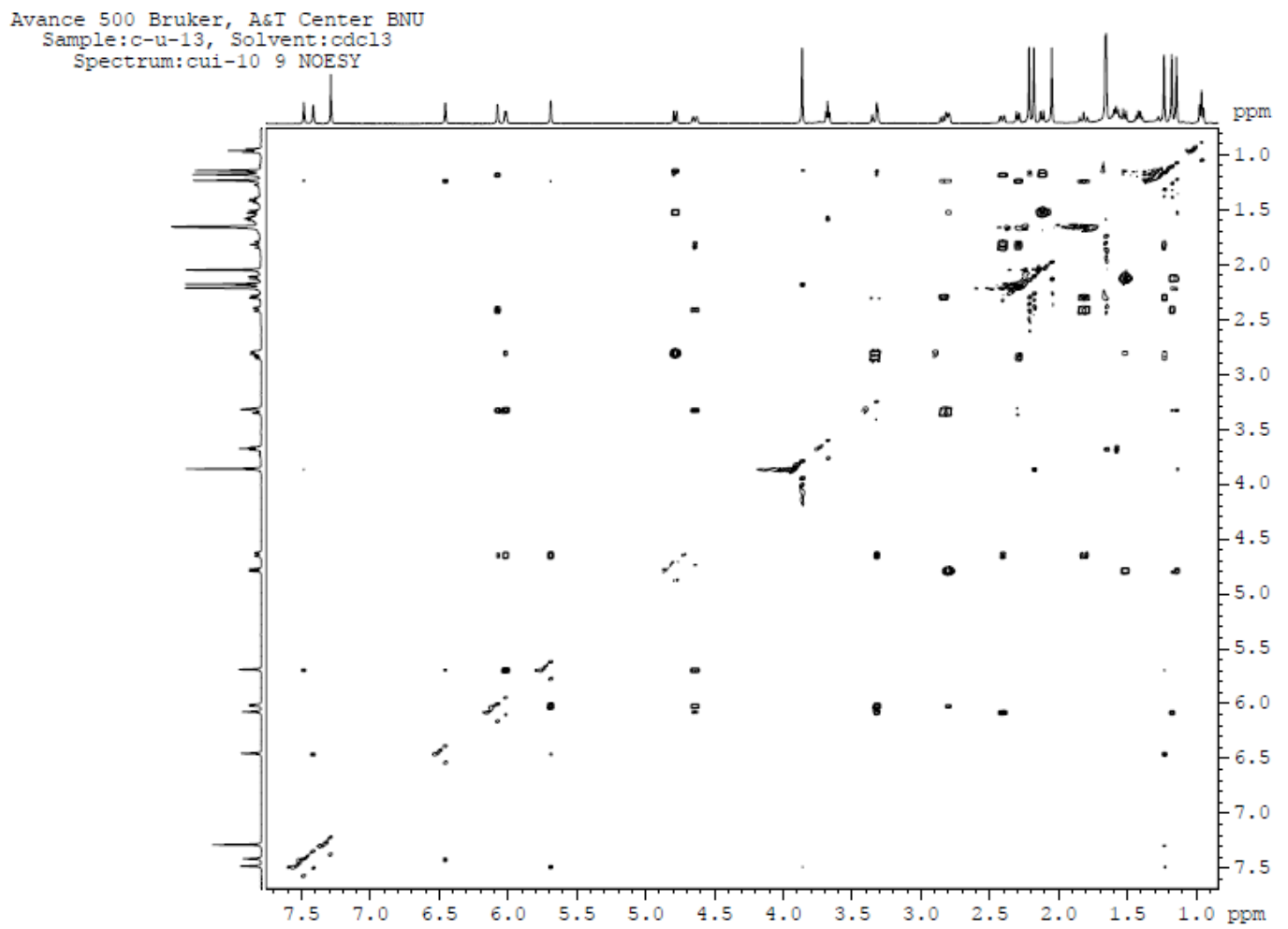

\section{ESIMS spectrum}

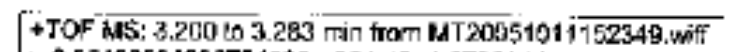

$*=35640250483975.46300004,10=4.879201491845045902+001$, subtracte.

M9x. 1237.6 counts

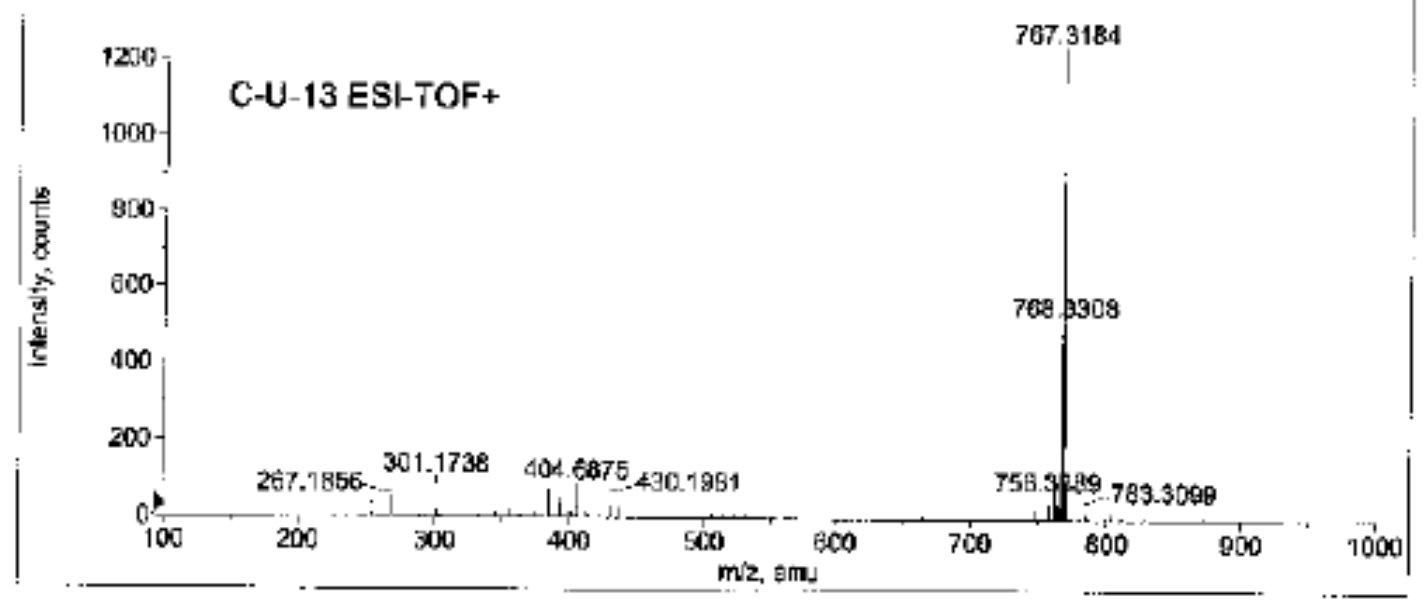




\section{HRSIMS data}

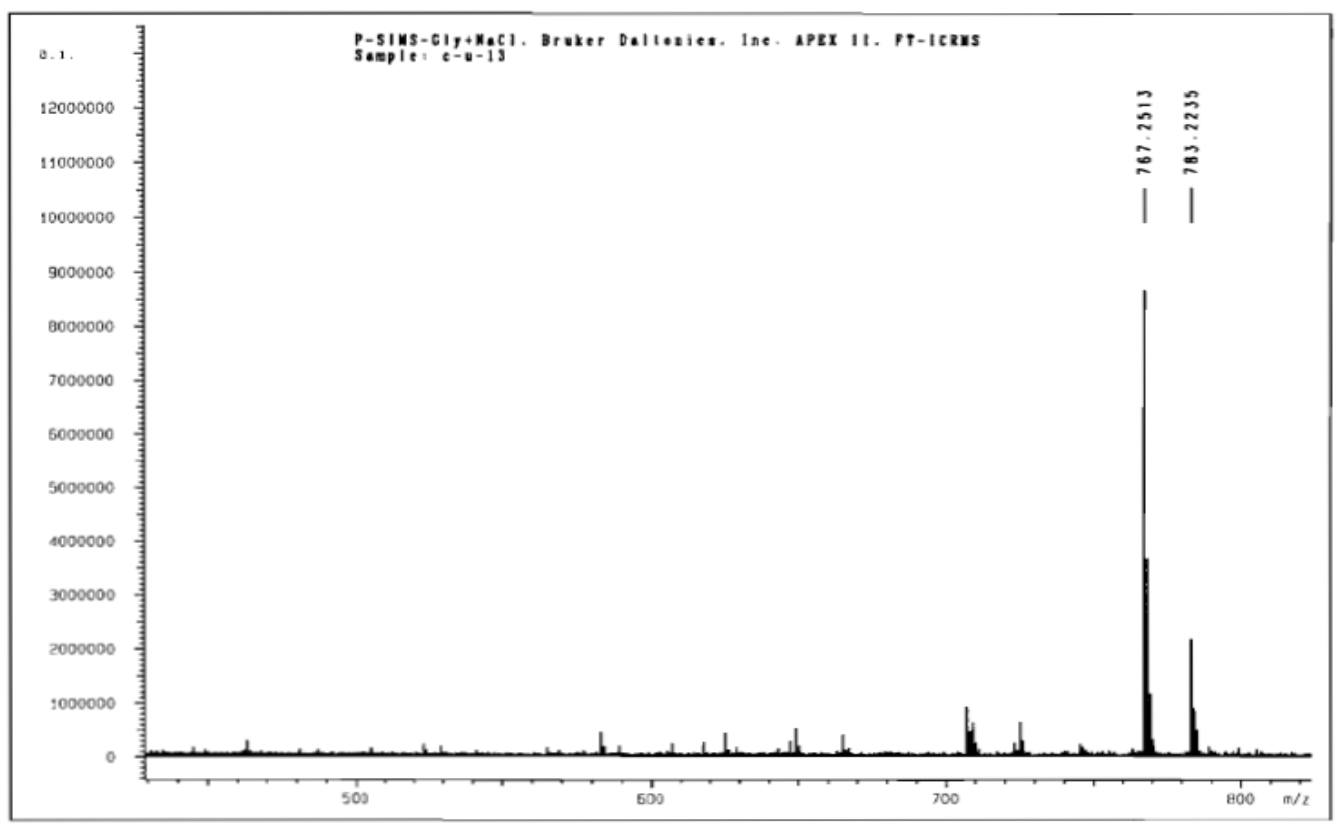

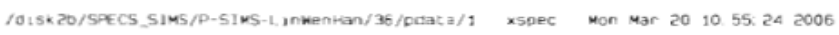

XMASS Mass Analysis for/disk2b/SPECS_SIMS/P-SIMS-WangYan/113/pdata/1/massanal XMASS Mass Analysis Constraints

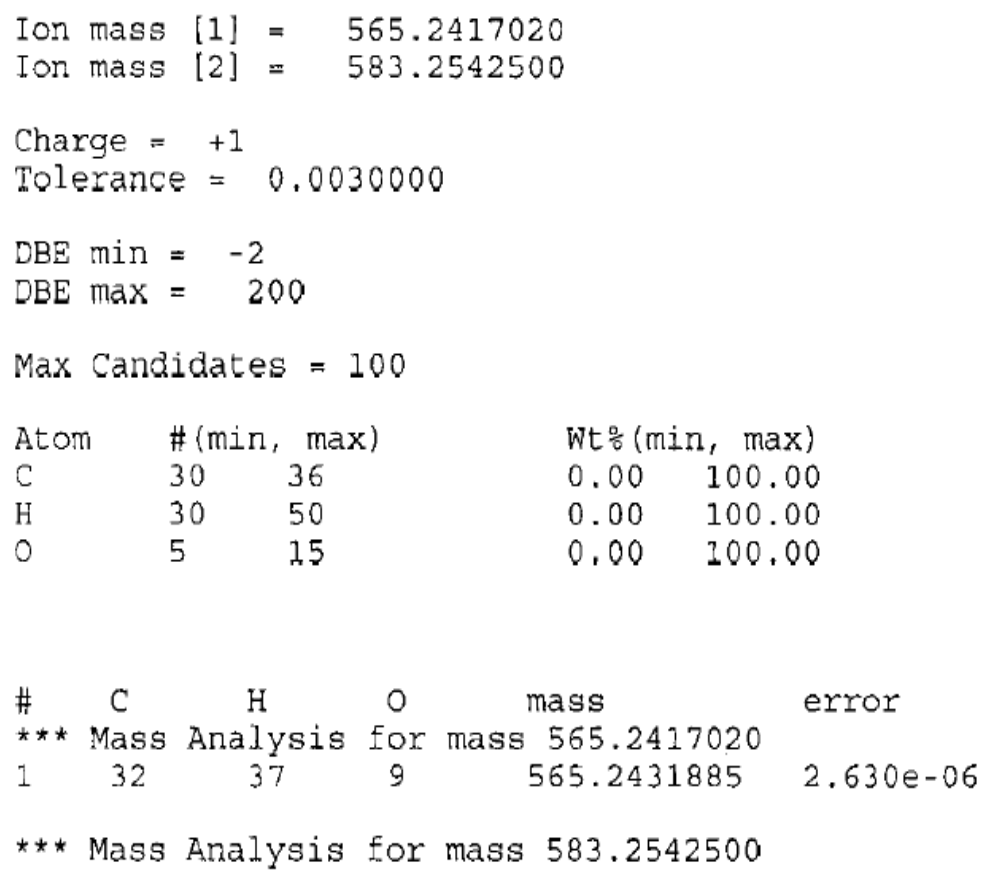



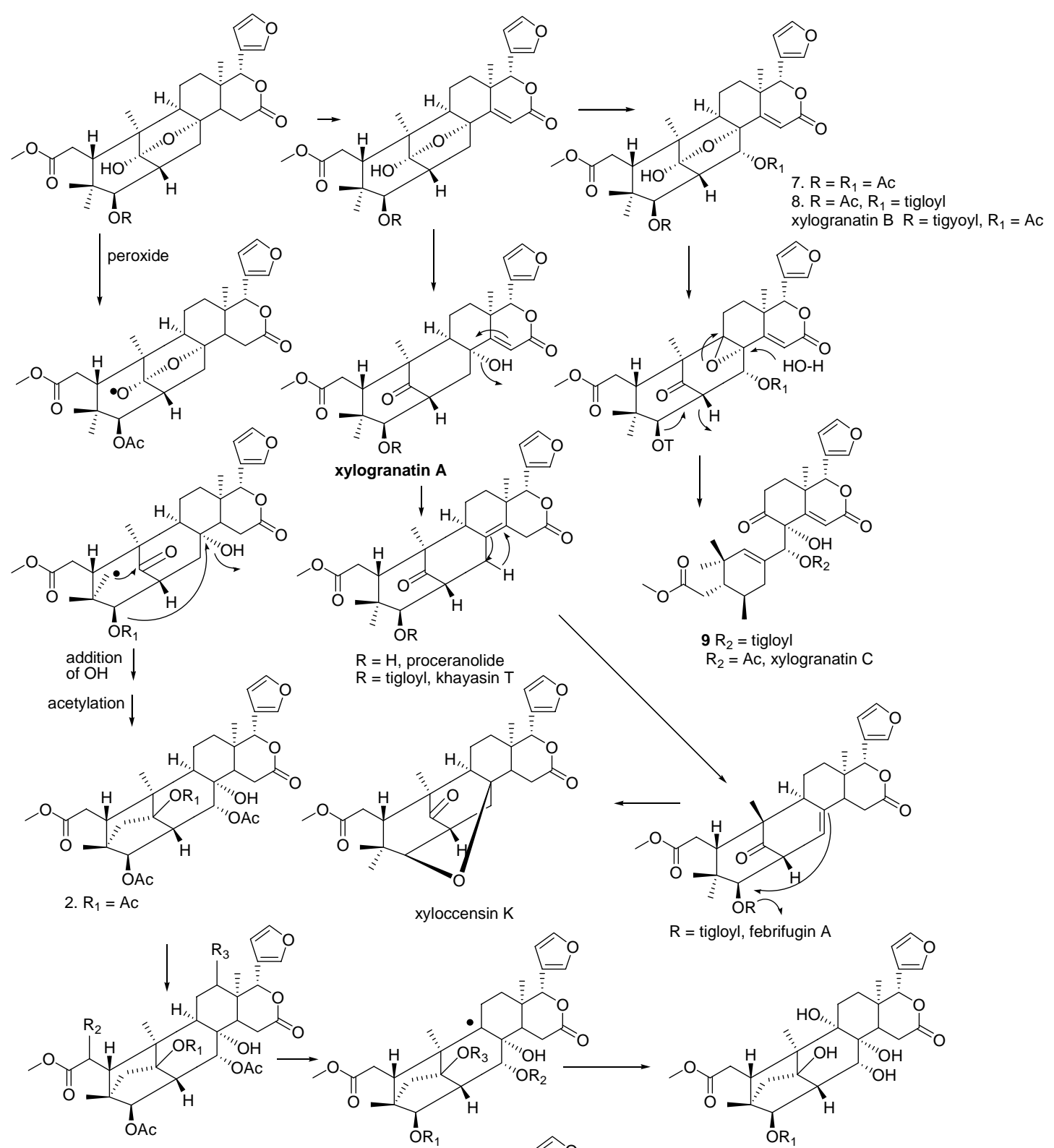

$R=$ tigloyl, febrifugin $A$

1. $R_{1}=A C, R_{2}=O A C, R_{3}=H$

3. $R_{1}=R_{2}=H, R_{3}=O A C$

4. $R_{1}=A C, R_{2}=O H, R_{3}=O A C$

5. $\mathrm{R}_{1}=A c, \mathrm{R}_{2}=\mathrm{OAc}, \mathrm{R}_{3}=\mathrm{OH}$

6. $R_{1}=A c, R_{2}=H, R_{3}=O A C$

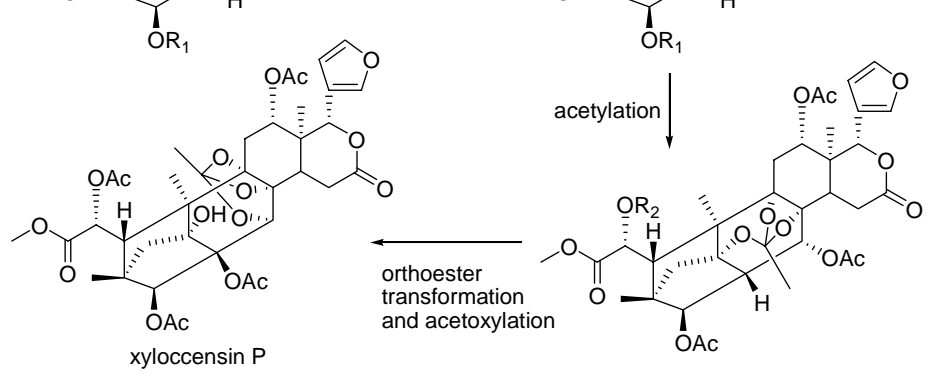

10

Scheme 1 The hypothesis of biotransformation of the isolated limonoids 